OPEN ACCESS

Edited by:

Alain Prochiantz,

Collège de France, France

Reviewed by:

Clotilde Thery,

Institut National de la Santé et de la

Recherche Médicale (INSERM),

France

Ulo Langel,

Stockholm University, Sweden

*Correspondence:

Teena K. J. B. Gamage

t.gamage@auckland.ac.nz

Specialty section:

This article was submitted to

Neurodevelopment,

a section of the journal

Frontiers in Neuroscience

Received: 21 July 2021

Accepted: 23 August 2021

Published: 24 September 2021

Citation:

Gamage TKJB and Fraser M (2021) The Role of Extracellular

Vesicles in the Developing Brain: Current Perspective and Promising Source of Biomarkers and Therapy

for Perinatal Brain Injury.

Front. Neurosci. 15:744840. doi: 10.3389/fnins.2021.744840

\section{The Role of Extracellular Vesicles in the Developing Brain: Current Perspective and Promising Source of Biomarkers and Therapy for Perinatal Brain Injury}

\author{
Teena K. J. B. Gamage* and Mhoyra Fraser \\ Department of Physiology, The University of Auckland, Auckland, New Zealand
}

This comprehensive review focuses on our current understanding of the proposed physiological and pathological functions of extracellular vesicles (EVs) in the developing brain. Furthermore, since EVs have attracted great interest as potential novel cell-free therapeutics, we discuss advances in the knowledge of stem cell- and astrocytederived EVs in relation to their potential for protection and repair following perinatal brain injury. This review identified 13 peer-reviewed studies evaluating the efficacy of EVs in animal models of perinatal brain injury; 12/13 utilized mesenchymal stem cell-derived EVs (MSC-EVs) and 1/13 utilized astrocyte-derived EVs. Animal model, method of EV isolation and size, route, timing, and dose administered varied between studies. Notwithstanding, EV treatment either improved and/or preserved perinatal brain structures both macroscopically and microscopically. Additionally, EV treatment modulated inflammatory responses and improved brain function. Collectively this suggests EVs can ameliorate, or repair damage associated with perinatal brain injury. These findings warrant further investigation to identify the optimal cell numbers, source, and dosage regimens of EVs, including long-term effects on functional outcomes.

\footnotetext{
Keywords: extracellular vesicles, exosomes, biomarkers, perinatal brain injury, white matter injury, in-vivo animal models, therapeutic strategies
}

\section{PERINATAL BRAIN INJURY}

Preterm infants born $<37$ weeks gestational age $(\mathrm{GA})$ are at a high risk of brain injury (Penn et al., 2016). Whilst improvements in the survival rates of preterm infants have occurred with advances in obstetric and neonatal care over the past two decades, infants that do survive are likely to experience some degree of long-term neurological impairment. Furthermore, it is the most immature of infants, namely the very preterm infants ( $28-<32$ weeks GA) and extremely preterm infants ( $<28$ weeks GA) who are at greater risk of lifetime disability and are estimated to account for 5.2\% of all preterm births <37 weeks GA (Beck et al., 2010; Blencowe et al., 2012; March of Dimes et al., 2012; Blencowe et al., 2013a).

Cerebral palsy $(\mathrm{CP})$ is the most common adverse neurodevelopmental outcome encountered by this most immature group of preterm infants. $\mathrm{CP}$ causes a range of permanent motor disabilities 
ranging from mild to severe. In the general population, the incidence of CP may be either increasing, static or declining amongst preterm infants (Vincer et al., 2006; Behrman and Butler, 2007; Platt et al., 2007; Robertson et al., 2007; van Haastert et al., 2011; Oskoui et al., 2013). Despite these incongruent trends, subsequent long-term adverse neurodevelopmental outcomes amongst preterm infants who survive constitute a major global health problem and is likely to escalate in the coming years given the increase in maternal age, increased access to assisted reproduction technology, and consequently multiple pregnancies worldwide (Ananth et al., 2005; Yeo et al., 2015; Wang et al., 2017).

Although the pathophysiological mechanisms that trigger injury to the preterm brain are multifactorial and the severity of injury is invariably a consequence of the degree of prematurity, there are several common causes. They include intrauterine infection (i.e., chorioamnionitis), which is recognized as an important factor in the etiology of spontaneous preterm birth and hypoxia-ischemia (HI), including postnatal insults, such as mechanical ventilation-induced lung injury, cerebral blood flow instability, free radical imbalance, sepsis, and necrotising enterocolitis (Stoll et al., 2004; Bassler et al., 2009; Martin et al., 2010; Polglase et al., 2012; Skiöld et al., 2014). Such perinatal

Abbreviations: ABCB1, ATP binding cassette subfamily member 1; AEA, Arachidonoylethanolamide; Akt, RAC-alpha serine/threonine-protein kinase; ALIX, ALG-2-interacting protein X; ANXA5, Annexin A5; ASC, apoptosisassociated speck-like protein containing a CARD; ATP, adenosine triphosphate; BAX, Bcl-2-associated X protein; BBB, blood-brain barrier; Bcl-XL, B-cell lymphoma extra-large; Bcl-2, B-cell lymphoma-2; BNIP2, BCL2 interacting protein 2; BDNF, brain derived neurotropic factor; CAT, catalase; CSF, cerebrospinal fluid; CNPase, 2',3,-cyclic nucleotide $3^{\prime}$-phosphodiesterase; CNS, central nervous system; COX, cyclooxygenase; CP, cerebral palsy; CREB, cAMP response element-binding protein; CTGF, connective tissue growth factor; CXCL10, chemokine (C-X-C motif) ligand-10; DAMP, damage associated molecular pattern; DT-MRI, diffusion tensor magnetic resonance imaging; ECG, electrocardiogram; EEG, electroencephalogram; EGR2, early growth response 2; EpCam, epithelial cell adhesion molecule; Erk1/2, extracellular signal-regulated kinases; GA, gestational age; GABA, gamma-aminobutyric acid; GAPDH, glyceraldehyde 3-phosphate dehydrogenase; GPX, glutathione peroxidase; HDAC1, histone deactelayse 1; HI, hypoxia-ischemia; HIE, hypoxicischemic encephalopathy; hiPSC-nEVs, human induced pluripotent stem cellderived EVs; HSF1, type 1 heat-shock factor; Iba1, ionized calcium binding adaptor molecule 1; IC, intracardial; ICAM, intercellular adhesion molecules; ICV, intracerebroventricular; IL, interleukin; iNOS, inducible nitric oxide synthase; IRDye, infrared dye; IV, intravenous; IVH, intraventricular hemorrhage; JAM1, junctional adhesion molecule-1; LPS, lipopolysaccharide; MAP2, microtubuleassociated protein 2; MBP, myelin basic protein; MDA, malondialdehyde; miRNA, microRNA; MISEV, minimal information for studies of extracellular vesicles 2018; MMP-9, matrix metallopeptidase-9; Matrix metallopeptidases (MMPs); MOG, myelin oligodendroglia glycoprotein; MRI, magnetic resonance imaging; MSC, mesenchymal stem cell-derived extracellular vesicles; NFK $\beta$, nuclear factor kappa beta; NRLP1, nod-like receptor protein 1; NTR, neutrophin receptor; OGD, oxygen-glucose deprivation; P, postnatal day; P2RX7, purinergic receptor P2X7; PDGF-BB, platelet-derived growth factor-BB; PDGFR $\beta$, platelet-derived growth factor receptor beta; PEG, polyethylene glycol; PLP, myelin proteolipid protein; PRISMA, Preferred Reporting Items for Systematic Reviews and MetaAnalyses; $\mathrm{PrP}^{\mathrm{C}}$, cellular prion protein; PTGDR, prostaglandin $\mathrm{D} 2$ receptor; $\mathrm{PTX}$, pentraxin 3; REST, restriction element-1 silencing transcription factor; RhoA, Ras homology family member A; STI1, stress-inducible protein 1; SEM, scanning electron microscopy; SOD, superoxide dismutase; SVZ, subventricular zone; TAG1, transiently expressed axonal surface glycoprotein-1; TEM, transmission electron microscopy; Timp3, tissue inhibitor of metalloproteinase 3; TkrB, tyrosine kinase receptor B; TNF, tumor necrosis factor; TRPS, tunable resistive pulse sensing; TSG101, tumor susceptibility gene 101; TTC, 2,3,5-tryphenyl tetrazolium chloride; TUNEL, terminal deoxynucleotidyl transferase dUTP nick end labeling. insults are intrinsically related to the development of a cascade of central and peripheral inflammatory processes, which play a critical role in the etiology of white matter injury, the most common injury observed in preterm infants. This pattern of brain damage is quite distinctive from that of term infants in that the lesions formed are often confined to the white matter tracts of the periventricular region of the brain, such as the corpus callosum, cingulum bundle, reticular activating system, and superior longitudinal fasciculi (Gopagondanahalli et al., 2016; Murray et al., 2016; van Tilborg et al., 2018). The primary pathological feature of this injury is the formation of focal cystic necrotic lesions and/or diffuse cerebral white matter injury accompanied by astrocytic hypertrophy (gliosis) and microglial activation. This can lead to a loss in white matter volume and impaired myelination, which is evident by magnetic resonance imaging (MRI) (van Tilborg et al., 2018).

While improved neonatal intensive care has resulted in a decline in the incidence of focal cystic necrotic lesions and thus the severest form of injury (Inder et al., 2003a), the incidence of "lesser" neurological morbidity associated with relatively milder forms of white matter injury remains an unresolved clinical issue (Behrman and Butler, 2007; Khwaja and Volpe, 2008; Blencowe et al., 2013b; Back, 2015). Typically, milder white matter injuries have a characteristic pattern featuring noncystic focal or diffuse white matter lesions within the periventricular region and surrounding white matter, which is invariably accompanied by some form of cortical or subcortical gray matter abnormality (Inder et al., 1999; Leviton and Gressens, 2007; Pierson et al., 2007; Keunen et al., 2012; Back and Miller, 2014; Galinsky et al., 2018). Further, converging evidence now suggests that the primary mechanism of myelination failure of the white matter tracts within 24-32 weeks GA, involves loss and subsequent arrested differentiation of oligodendrocyte progenitors, the predominant cell type in human white matter (Volpe et al., 2011; Buser et al., 2012; Back and Miller, 2014; van Tilborg et al., 2016). The more diffuse areas of cerebral white matter injury are characteristically identified as regions where there is a diffuse loss of developing oligodendrocytes and over time, clinically, these lesions are associated with smaller hemispheric size, ventriculomegaly, and impaired gyral development (Rutherford et al., 2010; Skranes et al., 2013; Alexandrou et al., 2014; Engelhardt et al., 2015).

Regardless of whether neurodevelopmental impairments encountered by preterm infants are either severe, moderate, or mild, the impact to the individual is enormous. It is not only a burden to the individual, but also immediate family members, the health care system, and social institutions. The development of effective therapies will enable better outcomes for these infants and thus have significant long-term benefits that could potentially translate to individuals achieving a more productive life including reduced health care costs associated with chronic neurological disability.

Presently, there are limited options for the prevention and/or treatment of perinatal brain injury. Treatment strategies include antenatal administration of magnesium sulfate to women at risk of preterm labor and postnatal administration of erythropoietin to infants born preterm (Leuchter et al., 2014; Juul and Pet, 2015; 
Crowther et al., 2017). However, these interventions are limited in their effectiveness. In addition, whilst multiple randomized trials suggest that therapeutic hypothermia initiated within the first $6 \mathrm{~h}$ of delivery of infants $\geq 36$ weeks GA with suspected moderate or severe hypoxic-ischemic encephalopathy (HIE) for a duration of $72 \mathrm{~h}$, reduces infant morbidity and mortality, it does not completely protect the infant from long-term brain damage (Gluckman et al., 2005; Azzopardi et al., 2009, 2014; Jacobs et al., 2011, 2013; Shankaran et al., 2012; Briatore et al., 2013). Furthermore, preterm infants born $<35$ weeks GA are not eligible for therapeutic hypothermia despite animal data supporting beneficial effects (Bennet et al., 2007). A pilot study of preterm infants concluded that therapeutic hypothermia was not recommended for preterm infants outside of a clinical research setting given the risk of mortality and side effects (Walsh, 2015). Nevertheless, a randomized controlled trial to assess safety and effectiveness of whole body hypothermia for $72 \mathrm{~h}$ in preterm infants 33-35 weeks GA who at $<6 \mathrm{~h}$ post-delivery exhibit moderate to severe neonatal encephalopathy is currently being conducted ( ${ }^{1}$ NCT01793129).

Whilst further randomized clinical trials of hypothermia therapy are warranted amongst preterm infants, there is an ongoing need to develop standalone therapeutic interventions that can be administered to reduce perinatal brain injury or therapeutic interventions that may be administered in conjunction with hypothermia to further reduce infant morbidity and mortality. Indeed, such an approach may be advantageous if the strategy is to target different therapeutic windows and multiple mechanisms of injury. However, hypothermia has the potential to alter the pharmokinetics of adjunct therapies and thus efficacy, which requires careful consideration (van den Broek et al., 2010; de Haan et al., 2012; Lutz et al., 2020).

Stem cells, which are self-renewing and considered the most primordial and least committed cells offer significant promise for treating perinatal brain injury (Kennea and Mehmet, 2004; Santner-Nanan et al., 2005; Vawda et al., 2007; Ikeda, 2008; de Paula et al., 2010; Pimentel-Coelho et al., 2010; PimentelCoelho and Mendez-Otero, 2010) given their anti-inflammatory (Li et al., 2005; Murphy et al., 2010; Kim et al., 2012), trophic (van Velthoven et al., 2012; Fu et al., 2017), and regenerative (Ilancheran et al., 2007; van Velthoven et al., 2010; Donega et al., 2014) capabilities. Indeed, neonatal models of hypoxic brain injury using different stem cell types support their therapeutic utility (van Velthoven et al., 2010, 2012; Kim et al., 2012; Donega et al., 2014) and presently there are several adult stroke clinical trials underway (e.g., clinical trials registry numbers: NCT02605707, NCT01151124, NCT04434768, NCT02980354). Despite their clinical potential, very little is known about their risk profile in a clinical neonatal setting. Such considerations are important given their identified potential to elicit unwanted immune responses and tumor formation or emboli (Lappalainen et al., 2008; Fischer et al., 2009; de Almeida et al., 2013).

Substantial evidence suggests that stem cells exert their regenerative effects through the release of biologically active molecules that act in a paracrine manner to promote cell viability

${ }^{1}$ ClinicalTrials.gov and/or proliferation and modulation of immune responses (Baraniak and McDevitt, 2010; Ratajczak et al., 2012). In fact, there has been a growing awareness among scientific and clinical arenas that stem cells are likely to promote neuroprotection and functional recovery via intercellular communication through their release of membrane bound vesicles, referred to as extracellular vesicles (EVs), which have a diverse array of bioactive cargo (Cho et al., 2019b). Therefore, the objective of this unbiased review was to provide an updated evaluation of EVs as therapeutic options in preclinical in-vivo animal models of perinatal brain injury.

\section{EVs}

The generic term "EVs" encompasses a heterogeneous range of lipid bilayered particles that are unable to replicate but are secreted by almost every cell of the body (Thery et al., 2018). Historically, EVs have been subdivided into (1) microvesicles (0.1-1 $\mu \mathrm{m}$ in size and often termed ectosomes), which bud from the plasma membrane of cells, (2) nanovesicles (30-150 nm in size and often termed exosomes; in this review they are referred to as "small" EVs), which are generated from late endosomes by inward budding of the limited multivesicular body membrane resulting in the formation of intraluminal vesicles within large multivesicular bodies that are transported to the cell surface and released into the extracellular environment, and (3) apoptotic bodies (1-5 $\mu \mathrm{m}$ in size), which originate from the plasma membranes of apoptotic cells (Pollet et al., 2018). However, there is some overlap in the size of EV subtypes and ambiguity remains between subtypes especially since their underlying intracellular biogenesis pathways cannot always be confirmed (Thery et al., 2018; Mathieu et al., 2019). Consequently, the recently released article "Minimal information for studies of extracellular vesicles 2018 (MISEV2018)" has encouraged researchers to describe EVs based upon (1) physical characteristics (2) biochemical composition, and/or (3) descriptions of conditions or cell of origin to help remove some of the ambiguity between EV subpopulations (Thery et al., 2018). Importantly, EVs carry a range of cargo, including nucleic acids [DNA, RNA, microRNA (miRNA), and non-coding RNAs] (Hunter et al., 2008; Simpson et al., 2008; Kalra et al., 2012; Gezer et al., 2014; Yoon et al., 2014; Abramowicz and Story, 2020; Born et al., 2020), proteins (Simpson et al., 2008; Cai et al., 2013), and lipids (Chen et al., 2019; Nishida-Aoki et al., 2020; Skotland et al., 2020) to local and distal targets that can impact the biology of the target cells by conveying functional properties derived from their cellular source (Cocucci and Meldolesi, 2015; Campanella et al., 2019).

\section{EV-Mediated Cell Communication and Roles Within the Healthy Brain}

All major cell types of the brain, including oligodendrocytes, neurons, microglia, astrocytes, endothelial cells and pericytes secrete EVs (Fevrier et al., 2004; Bakhti et al., 2011; Fitzner et al., 2011; Frühbeis et al., 2013b; Lewis, 2013; Sharma et al., 2013; Prada et al., 2018). A number of stimuli can trigger the release of EVs from neuronal cells. For example, synaptic transmission resulting from calcium influx and glutamatergic synaptic activity 
elicits secretion of neuron-derived EVs carrying the $\alpha$-amino3-hydroxy-5-methyl-4-isoxazolepropionic acid (AMPA) receptor subunit (Budnik et al., 2016). Moreover, glutamate released from neurons triggers $\mathrm{EV}$ release from oligodendrocytes through $\mathrm{Ca}^{2+}$ entry via oligodendroglial ionotropic glutamate receptors (Frühbeis et al., 2013b).

Although the functional role of the various cell types of EVs within the central nervous system (CNS) is not fully ascertained those identified as being mediated by EV autocrine/paracrine signaling include neural trophic support, synaptic plasticity, regulation of myelination and intercellular communication (Coleman and Hill, 2015; Budnik et al., 2016; Thompson et al., 2016). Neuron-derived EVs have been demonstrated to play a critical role in neurite elongation, a prerequisite step required for the assembly of adult neurons in functional networks (Arantes and Andrews, 2006). Human primary neural cultures treated with human induced pluripotent stem cell-derived neuron EVs (hiPSC-nEVs) results in increased cell proliferation and neuronal cell fate specification and differentiation (Sharma et al., 2019). Additionally, data from preclinical studies, in which hiPSCnEVs were injected into the lateral ventricle of postnatal day 4 (P4) mice, demonstrate their role in hippocampal neurogenesis (Sharma et al., 2019). Synaptic plasticity is also thought to be mediated by EVs that are known to play roles in synaptic growth (Korkut et al., 2013) and axonal guidance (Gong et al., 2016), as well as synaptic pruning (Bahrini et al., 2015).

Development of the neurocircuitry, is partly mediated through neuronal-EV interactions with glial cells. For example, the uptake of oligodendrocyte derived-EVs by neuronal cells can enhance neuronal cell viability though RAC-alpha serine/threonine-protein kinase (Akt), Extracellular signalregulated kinases (Erk1/2) and cAMP response element-binding protein (CREB) pathway activation and generate more action potentials suggesting an enhanced potential for neuronal signaling (Fröhlich et al., 2014). Furthermore, the uptake of astrocyte-derived EVs that carry co-chaperone stress-inducible protein 1 (STI1) by neuronal cells can have neurotrophic and neuroprotective roles upon binding to cellular prion protein $\left(\operatorname{PrP}^{\mathrm{C}}\right)$ (Hajj et al., 2013). Additionally, dendrite complexity can be enhanced in neurons as a result of the miR-26a-5p cargo of astrocyte-derived EVs (Polanco et al., 2016). The activity of these neuronal networks can also be regulated by microglia-derived EVs that can target neurons where they can act both by stimulating synaptic activity through enhancing sphingolipid metabolism (Emmanouilidou et al., 2010) or inhibiting gamma-aminobutyric acid (GABA)-ergic transmission via $\mathrm{N}$-arachidonoylethanolamine (AEA) signaling (Danzer et al., 2012). Conversely, neuronal-derived EVs can also directly target microglia to stimulate complement C3 activity which aids synaptic pruning (Bahrini et al., 2015).

EVs can also play a significant role in blood-brain barrier (BBB) integrity. Platelet-derived growth factor-BB (PDGFBB)/PDFG-receptor beta (PDGFR- $\beta$ ) signaling has been implicated in the release of pericyte-derived EVs that carry neuroprotective cargo (Prada et al., 2018). This is particularly relevant in the context of ischemic conditions, since PDGF$\mathrm{BB} / \mathrm{PGDFR} \beta$ signaling is known to confer protection of the
$\mathrm{BBB}$ and provide regeneration of infarcted regions by way of enhancing pericyte recruitment (Nakamura et al., 2016). Evidence also suggests that neural progenitor cell-derived EVs enhance post-ischemic BBB integrity by enhancing pericyte recruitment via down-regulation of ATP binding cassette subfamily member 1 (ABCB1) expression and inhibition of the nuclear factor-kappa beta (NF- $\mathrm{k} \beta$ ) pathway and downstream matrix metallopeptidase-9 (MMP-9) activity (Zhang et al., 2021).

Oligodendrocytes, the myelin-forming cells, are well established as having important neuron-protecting roles. Indeed, there is well-founded evidence that oligodendroglial-derived EVs, besides being enriched with myelin proteins [myelin proteolipid protein (PLP), 2', 3'-cyclic nucleotide $3^{\prime}$-phosphodiesterase (CNPase), myelin associated glycoprotein, and myelin oligodendroglial glycoprotein (MOG)] are mediators of oxidative stress through transfer of human superoxide dismutase (SOD) and catalase (CAT) (Krämer-Albers et al., 2007). Furthermore, neuronal uptake of oligodendrocyte-derived EVs under in-vitro conditions of oxygen and glucose can modulate neuronal firing rate and is associated with enhanced stress resistance and neuronal viability (Frühbeis et al., 2013a; Fröhlich et al., 2014).

The uptake of oligodendrocyte-derived EVs by microglia, the resident macrophage, occurs by way of macropinocytosis, which facilitates the clearance of oligodendrocyte-derived EV cargo and occurs in the absence of microglial activation and without induction of a $\mathrm{CD} 4^{+} \mathrm{T}$ cell response to MOG (Fitzner et al., 2011). However, in pathogenic situations, such as multiple sclerosis, EVs exposed to phagocytic microglia may induce activation of microglia or possibly even evoke signaling to $\mathrm{T}$ - and $\mathrm{B}$-cells, which have invaded the CNS via the BBB (Dolcetti et al., 2020). Furthermore, and of particular relevance to the current review, oligodendrocyte-derived EV cargo can act in an autocrine manner to inhibit oligodendrocyte maturation (Bakhti et al., 2011), whereas BBB endothelial cellderived EVs aid oligodendrocyte precursor survival, motility, and proliferation (Fevrier et al., 2004). Consequently, the clearance of oligodendrocyte-derived EV cargo by microglia in the presence of neuronal cells may allow immature pre-myelinating oligodendrocytes to mature into myelinating oligodendrocytes and thus promote the establishment of myelinated neural circuitry (Bakhti et al., 2011).

Collectively, the above evidence suggests an intricate, delicate, and highly co-ordinated interplay between EVs and all cell types of the brain that has important implications for the establishment and maintenance of a healthy neural circuitry (Figure 1).

\section{The Role of EVs in CNS-Immune Communication Following Injury}

Over the last two decades, significant emphasis has been placed on understanding the process and timing of injury in the developing brain to improve therapeutic interventions. Seminal studies conducted in animal models have led to the realization that brain injury is an evolving process involving two phases. First there is a latent phase of recovery lasting approximately $6-8 \mathrm{~h}$, followed by a delayed or secondary phase of injury characterized by mitochondrial failure, with seizures 


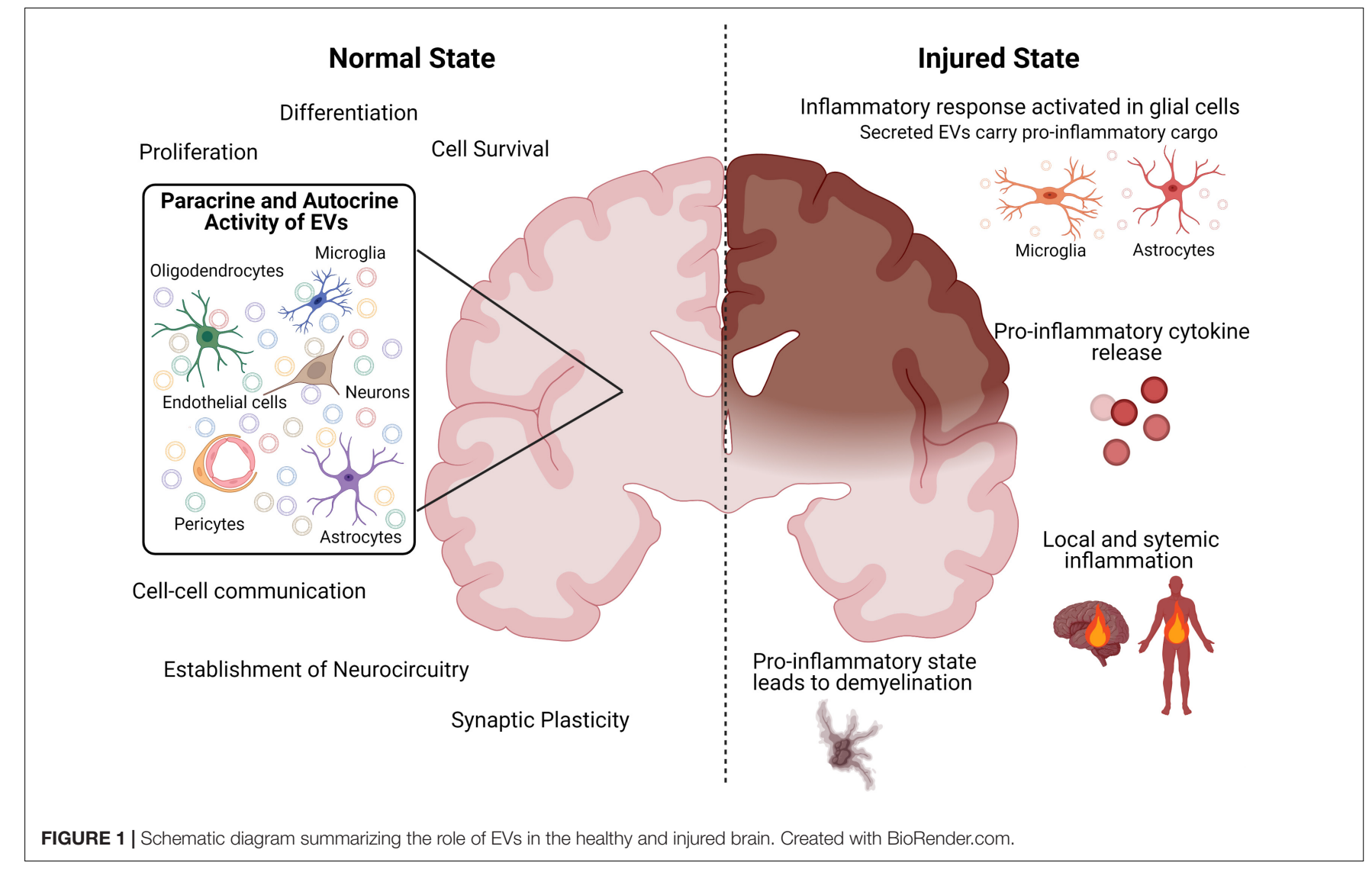

and cell swelling that resolves after $72 \mathrm{~h}$ (Williams et al., 1991; Fraser et al., 2005, 2007, 2008).

While the etiology of preterm white matter injury is multifactorial, inflammation plays an important role in the pathogenesis of preterm brain injury (Rezaie and Dean, 2002; Deng et al., 2010; Jellema et al., 2013a; Albertsson et al., 2014; Mallard et al., 2014; Dhillon et al., 2015; Hagberg et al., 2015). The ensuing neuroinflammatory cascade following an insult comprises a wide array of both humoral and cellular players. Microglia, the resident macrophage population in the CNS, along with astrocytes, transform into activated cells that migrate or extend their processes, respectively, to sites of injury. Once activated, these cells produce potentially damaging proinflammatory mediators, such as pro-inflammatory cytokines [tumor necrosis factor-alpha (TNF- $\alpha$ ), interleukin-1 $\beta$ (IL-1 $\beta$ ), and interleukin-6 (IL-6)], enzymes and adhesion molecules, including the release of matrix metallopeptidases (MMPs), which lead to the breakdown of the immature BBB (Lau and Yu, 2001; Ranasinghe et al., 2009, 2012; Iadecola and Anrather, 2011; Baburamani et al., 2014). In response to this inflammatory activation, leukocytes migrate through the $\mathrm{BBB}$ leaving the brain exposed to systemic inflammatory responses, which further exacerbate injury. Collectively, this results in the progressive destruction of white matter and surrounding CNS tissue.

Modulation of the CNS and peripheral inflammatory response in relation to the timing of the initial insult is most likely key to effective therapeutic targeting. There is a clear recognition from animal models of brain injury that inhibition of EV release from the CNS attenuates systemic responses to CNS inflammation and inhibits BBB leukocyte infiltration, suggesting a damaging role of EVs (Dickens et al., 2017). Indeed, damageassociated molecular pattern (DAMP)-mediated activation with adenosine triphosphate (ATP), results in the release of EVs from microglia (Drago et al., 2017). Specifically, ATP results in modifications of the proteome of EVs derived from microglia responsible for the synthesis of proteins involved in cellular adhesion/extracellular matrix organization, the autophagylysosomal pathway and cellular metabolism that can modulate astrocyte activity (Drago et al., 2017). Furthermore, in an IL-1 $\beta$ mouse model of inflammatory brain injury, astrocyticderived EVs released post-injury are able to induce a systemic inflammatory response in naive animals, in the absence of injury (Figure 1; Dickens et al., 2017).

Microglia-derived EVs appear to play an equally detrimental role in promoting a pro-inflammatory microenvironment response to brain injury (Figure 1). Importantly, recent findings suggest that EVs released from microglia in response to brain injury may represent the major pathway of TNF- $\alpha$ secretion, since EV production is markedly induced by activation of the purinergic receptor P2X7 (P2RX7) by ATP (Bianco et al., 2009). There is also evidence that ATP activation of microglia results in the release of EVs with IL-1 $\beta$ and glyceraldehyde 3-phosphate dehydrogenase (GAPDH) that facilitate the propagation and regulation of the neuroinflammatory response in the brain 
(Bianco et al., 2005; Takenouchi et al., 2015). In this respect, EVs produced by activated microglia contain high amounts of TNF- $\alpha$ and can induce reactive astrocytic conversion and demyelination (Figure 1; Lombardi et al., 2019).

Clearly redirecting or reprogramming inflammatory glial cells by way of modifying EV signaling toward a beneficial and proregenerative function might facilitate repair processes following injury. It would be important also to delineate the origin of EVs in the circulation released in response to injury as they may provide much needed information on possible key facilitators of CNSperipheral communication and thus identify a specific cellular target for EV therapy.

\section{Biomarker Potential of CNS-Derived EVs}

In addition to the above established and putative roles of EVs within the brain, accumulating evidence suggests that the number and composition of EVs can reflect both healthy and pathological states within the CNS (Fiandaca et al., 2015; Yan et al., 2017; Goetzl et al., 2019; Ohmichi et al., 2019). Although investigations are yet in their infancy, evidence suggests that EVs are found throughout the developing CNS (Morton and Feliciano, 2016) and are likely responsible, at least in part, for normal and pathophysiological brain development in the embryonic and early fetal periods. In support of the former, embryonic cerebrospinal fluid (CSF)-derived small EVs promote neuronal stem cell proliferation possibly through miRNA-mediated regulation of the insulin growth factor pathway (Feliciano et al., 2014; Tietje et al., 2014; Luarte et al., 2016). In addition, small EVs derived from progenitor cells may have protective roles in neuronal development, protecting against such things as hypoxia mediated apoptosis (Deng et al., 2018; Guo et al., 2019; Liu et al., 2019). Furthermore, EVs released by neural stem cells residing in the neonatal subventricular zone (SVZ), lining the lateral ventricles, have the capacity to regulate microglial function as they are enriched with miRNAs (miR-9 and miR-Let7) that regulate microglial morphology (Yao et al., 2014; Morton et al., 2018; Vogel et al., 2018). Indeed, according to novel findings, neonatal SVZ neural stem cellderived EVs can preferentially target microglia and regulate their morphological and physiological function, suggesting they play important roles within the developing brain (Morton et al., 2018). However, while many of the ascribed EV-mediated effects are foreseen as beneficial they can also be detrimental and under pathological conditions, damaged and distressed cells can release EVs carrying altered protein, lipid, and nucleic acid cargos that may potentially exacerbate the injury (Rajendran et al., 2006; Banigan et al., 2013; Zagrean et al., 2018). Specifically, pathological cells can release EVs that can spread neurological disease and this is most evident in neurodegenerative diseases (Lee and Kim, 2017). For example, cells of Alzheimer's patients brains are known to release neurotoxic amyloid- $\beta$ between cells via EVs, which is one mechanism by which disease progression and propagation is thought to occur (Rajendran et al., 2006; Joshi et al., 2014). Similarly, the presynaptic neuronal protein $\alpha$-synuclein associated with Parkinson's disease and various other neurodegenerative diseases is transmitted between cells in an EV-mediated manner and drastically impairs neuronal cell viability (Emmanouilidou et al., 2010; Danzer et al., 2012). Moreover, EVs isolated from the CSF of individuals with spinal cord injury and traumatic brain injury express inflammasome proteins, including caspase, apoptosis-associated speck-like protein containing a CARD (ASC), and nod-like receptor protein 1 (NRLP1), suggesting that EVs released in response to CNS damage may be involved in activation of inflammatory signaling processes (de Rivero Vaccari et al., 2016). Consequently, it is equally plausible that EVs released in response to perinatal brain injury may also contribute to the evolution of brain injury pathology over time.

A recently revealed key characteristic of small EVs derived from the fetal CNS is that they are able to cross the fetal $\mathrm{BBB}$ and the placental barrier into the maternal circulation making them an attractive source of biomarkers (ShellerMiller et al., 2016; Goetzl et al., 2019). Adsorptive-mediated transcytosis, translocation and passage from the CSF via arachnoid granulations are all proposed mechanisms of small EV transfer from the CNS into the periphery (Thompson et al., 2016; Matsumoto et al., 2017). However, evidence currently suggests that fetal CNS-derived small EVs do not fuse with the placental membrane in order to cross into the maternal circulation, and further studies are required to clarify the mechanisms of fetal CNS-derived small EV transfer into the maternal circulation (Goetzl et al., 2019). Nevertheless, 20\% of total neuronal cellderived small EVs isolated from first and second trimester maternal plasma samples are comprised of fetal CNS-derived small EVs, which suggests the possibility that their cargo may be possible biomarkers of preterm brain injury (Goetzl et al., 2016).

Investigations have been conducted to assess whether fetal CNS-derived small EVs isolated from maternal plasma during pregnancy can feasibly detect adverse fetal neurodevelopmental outcomes resulting from ethanol exposure as early as the first trimester (Goetzl et al., 2016, 2019). Specifically, the authors of these studies were able to isolate fetal CNS-derived EVs using an antibody raised against Contactin-2/transiently expressed axonal surface glycoprotein-1 (TAG-1), which is expressed transiently on the axonal surface of specific neurons during fetal life (Goetzl et al., 2019). Analysis of fetal CNS-derived EV cargo has revealed that expression of miR-9, a key microRNA involved in neurogenesis (Coolen et al., 2013), and protein levels of synaptic markers neurogranin, synaptotagmin, synatopodin and synaptophysin are all significantly reduced in maternal plasma in association with ethanol exposure (Goetzl et al., 2019). Moreover, levels of neuronal survival proteins [type 1 heatshock factor (HSF1), B-cell lymphoma extra-large (Bcl-XL) and restriction element-1 silencing transcription factor (REST)] are also significantly lower (Goetzl et al., 2016).

Furthermore, studies conducted to assess whether small $\mathrm{EV}$ protein biomarkers are valuable in the diagnosis of brain injury and assessment of the effectiveness of hypothermia have shown that neutral or decreasing fetal neuronal small EV-derived synaptopodin protein levels occurred in maternal plasma of neonates with abnormal neuroimaging scores (Goetzl et al., 2017). Interestingly, evidence from one preterm infant in which CSF-derived small EVs were collected over the course of 4 months following post-hemorrhagic hydrocephalus, there 
was a reduction in the concentration and proportion of EVs within the 30-100 $\mathrm{nm}$ range, but the physiological significance of this is uncertain (Spaull et al., 2019). Together, the studies discussed above are representative of an ever-growing awareness that screening EV profiles of either CSF and plasma of preterm infants or that of their mother's plasma during pregnancy could be a means to provide early diagnosis of preterm brain injury and ultimately effective implementation of treatment strategies. Furthermore, since EVs can also cross the BBB in the opposite direction (Chen et al., 2016; Mondello et al., 2018; Saint-Pol et al., 2020), their entry into the brain opens up the possibility of harnessing their innate neuroprotective or restorative functions or engineering them for delivery of drugs or biomolecules for the treatment of perinatal brain injury (Cho et al., 2019b).

\section{EVs as a Therapeutic Agent for Perinatal Brain Injury}

In-vitro evidence suggests that small EVs have promising neuroprotective and neuroregenerative effects that could be utilized for the treatment of brain injury. Specifically, in a mouse neuroblastoma cell line Neuro-2a model of $\mathrm{HI}$ induced by oxygen-glucose deprivation (OGD)/reoxygenation, administration of human umbilical cord Wharton's jelly MSCEVs not only resulted in the prevention of HI-induced apoptosis, but also promoted cell survival through EV transfer of miR-let7-5p, a known regulator of caspase-3 (JoergerMesserli et al., 2018). In addition, researchers recently reported the effect of human Wharton's jelly MSC-EVs on microgliamediated neuroinflammation. They observed that MSC-EVs reduced pro-inflammatory cytokine production in response to lipopolysaccharide (LPS) from activated microglia in-vitro (Thomi et al., 2019b). Moreover, the treatment of primary rat neurons exposed to thrombin [as a surrogate model of HI with intraventricular hemorrhage (IVH)] with human umbilical cord blood MSC-EVs attenuated cell death (Ahn et al., 2021).

While the majority of in-vitro evidence demonstrates EVs from a variety of cell sources have great potential as both neuroprotective and neuroregenerative agents (Doeppner et al., 2015; Jarmalavičiūtè et al., 2015; Bonafede et al., 2016), more information is required from relevant in-vivo experimental models of perinatal brain injury before consideration of translation to the clinic. Theoretically, EVs may be biologically safer over cell based therapies as they are potentially less likely to induce an immunogenic response, cause tumor formation, or cause thrombosis in the recipient and in practice easier to collect, store and administer (Adamiak et al., 2018; Ma et al., 2019). However, longitudinal studies are necessary to validate such an assumption. Furthermore, recent data from an in-vitro model system demonstrating $\mathrm{HI}$ injured neuronal cells potentially can secrete EVs that may contribute to cell death of healthy MSC cells, is of relevance since this could impact on the efficacy of cell based therapies depending on the timing of administration post-injury (Huang et al., 2020).

Current evidence suggests EVs secreted by cells exhibit target selection and that the EV cellular source must be matched to the requirements of the target application
(Marcus and Leonard, 2013). In this respect, there is an increasing awareness that generation of neural stem cell-derived EVs would more likely exhibit the highest distribution to the brain and confer neuroprotective benefits. Furthermore, successfully translating interventions from preclinical proof of concept to demonstration of therapeutic value in the clinic requires a route of administration that is minimally invasive, yet effective, such as intranasal delivery, which has a greater likelihood of achieving therapeutic levels. Recent studies of intranasal administration of EVs to neonatal and juvenile rat models of brain injury support the potential clinical relevance of this approach, since EVs targeted a diverse range of brain regions and aggregated to areas of injury (Kodali et al., 2019; Thomi et al., 2019a).

Moving forward, it is apparent the use of well-characterized EVs according to the most recent MISEV guidelines (Thery et al., 2018), produced under specific culture conditions and isolated appropriately to ensure EV integrity, have great promise for treating perinatal brain injury. Consequently, in this unbiased review of the literature the focus was to identify and summarize current evidence of the role of EVs administered to in-vivo models of perinatal brain injury as a therapeutic strategy.

\section{Methods: Search Strategy, Study Selection and Data Extraction}

An unbiased search of the literature was conducted using the Preferred Reporting Items for Systematic Reviews and MetaAnalyses (PRISMA) to identify all studies that investigated the potential use of EVs as novel therapeutic agents in animal models of perinatal brain injury (the PRISMA checklist is provided in Supplementary File 1). Eligible studies were identified by searching four databases (PubMed, Embase, Scopus and Web of Science). Keywords included in the search were: perinatal brain injury and extracellular vesicles, perinatal brain injury and exosomes, perinatal hypoxic ischemia and extracellular vesicles, perinatal hypoxic ischemia and exosomes, perinatal encephalopathy and extracellular vesicles, perinatal encephalopathy and exosomes, perinatal brain injury and biomarkers, preterm brain injury and extracellular vesicles, preterm brain injury and exosomes, preterm hypoxic ischemia and extracellular vesicles, preterm hypoxic ischemia and exosomes, preterm encephalopathy and extracellular vesicles, preterm encephalopathy and exosomes, preterm brain injury and biomarkers, fetal brain injury and extracellular vesicles, fetal brain injury and exosomes, fetal hypoxic ischemia and extracellular vesicles, fetal hypoxic ischemia and exosomes, fetal encephalopathy and extracellular vesicles, fetal encephalopathy and exosomes, fetal brain injury and biomarkers, neonatal brain injury and extracellular vesicles, neonatal brain injury and exosomes, neonatal hypoxic ischemia and extracellular vesicles, neonatal hypoxic ischemia and exosomes, neonatal encephalopathy and extracellular vesicles, neonatal encephalopathy and exosomes, and neonatal brain injury and biomarkers. The publication search was conducted on 1 April 2021 and publication dates ranged as far back as 1977. Filters were not applied to any database used in this review of the 
literature to ensure all relevant papers were captured; instead, articles were manually filtered.

A total of 30,965 articles were identified and imported into Endnote X7 referencing management software and 17,159 duplicate records removed. The remaining 13,806 articles were analyzed by title and abstract and 13,793 irrelevant articles removed based on non-English, non-full text, non-original article, and off-topic subject matter. Where ambiguity was identified in the title and abstract, the articles were read in full to identify whether the use of EVs as a therapeutic for perinatal brain injury using animal models was investigated. Thirteen articles were identified as relevant for this literature review (Figure 2 and Table 1) based upon this premise.

\section{RESULTS}

\section{Summary of Therapeutic EV Studies in Experimental Animal Models of Perinatal Brain Injury}

Thirteen studies that utilize EVs as a therapeutic intervention for the treatment of perinatal brain injury using an in-vivo model were identified in this unbiased review of the literature (Figure 2 and Table 1; Ophelders et al., 2016; Drommelschmidt et al., 2017; Shu et al., 2018; Gussenhoven et al., 2019; Sisa et al., 2019; Thomi et al., 2019a,b; Chu et al., 2020; Han et al., 2020; Kaminski et al., 2020; Xin et al., 2020; Ahn et al., 2021; Du et al., 2021). Eight of these studies used models of $\mathrm{HI}$ alone (Ophelders et al., 2016; Gussenhoven et al., 2019; Sisa et al., 2019; Chu et al., 2020; Han et al., 2020; Kaminski et al., 2020; Xin et al., 2020; Du et al., 2021), three studies used models of simulated infection in the setting of HI (Drommelschmidt et al., 2017; Thomi et al., 2019a,b), one study utilized a model of IVH (Ahn et al., 2021) and one study did not provide sufficient details within the publication to determine the model of brain injury (Shu et al., 2018; Table 1). 12/13 studies utilized MSC-derived EVs. However,
8/12 studies utilized EVs produced by bone marrow-derived MSCs (Ophelders et al., 2016; Drommelschmidt et al., 2017; Shu et al., 2018; Gussenhoven et al., 2019; Sisa et al., 2019; Chu et al., 2020; Kaminski et al., 2020; Xin et al., 2020). Of the eight perinatal brain injury studies which utilized bone marrow-derived MSCEVs, only one study administered rat MSC-EVs in a rat model (Shu et al., 2018), two studies administered mouse MSC-EVs in a mouse model (Chu et al., 2020; Xin et al., 2020), whereas all other studies utilized human bone marrow-derived MSC-EVs on either a rat (Drommelschmidt et al., 2017), mouse (Sisa et al., 2019; Kaminski et al., 2020) or fetal sheep (Ophelders et al., 2016; Gussenhoven et al., 2019) model (Table 1 and Figure 3). In addition, 2/12 studies utilized human umbilical cord Wharton's jelly derived MSCs-EVs in a rat model (Thomi et al., 2019a,b), $1 / 12$ studies utilized human umbilical cord blood MSC-EVs in a rat model (Ahn et al., 2021) and 1/12 studies utilized umbilical cord-derived MSC-EVs in a mouse model (Han et al., 2020; Table 1 and Figure 3). Interestingly, one study, which met the eligibility criteria used hydrogen sulfide to pretreat MSCs in order confer a neuroprotective phenotype in MSC-EVs. In this study, hydrogen sulfide treatment enriched the MSC-EVs with miR-7b$5 \mathrm{p}$, a miRNA the authors hypothesized as being responsible for exerting the therapeutic effects of MSC-EVs (Chu et al., 2020). Given the diversity of EV sources utilized in these studies, it warrants mentioning that EVs from different MSC sources may have variable therapeutic potential, since small EVs from human umbilical cord MSCs appear to be most effective in gynecological and perinatal conditions (Cai et al., 2020). Finally, 1/13 studies identified in this literature search utilized rat astrocyte-derived EVs in a neonatal rat model of HI (Table 1 and Figure 3; Du et al., 2021).

In all studies identified, centrifugation (either low speedor ultra-centrifugation) was employed (either alone or with an additional purification technique) to isolate EVs ranging from 16.34 to $1000 \mathrm{~nm}$ (Table 1). Depending on the study, EVs stained positive for a range of membrane protein markers [CD63, CD81, CD9, Flotillin-1, Epithelial cell adhesion molecule (EpCam),

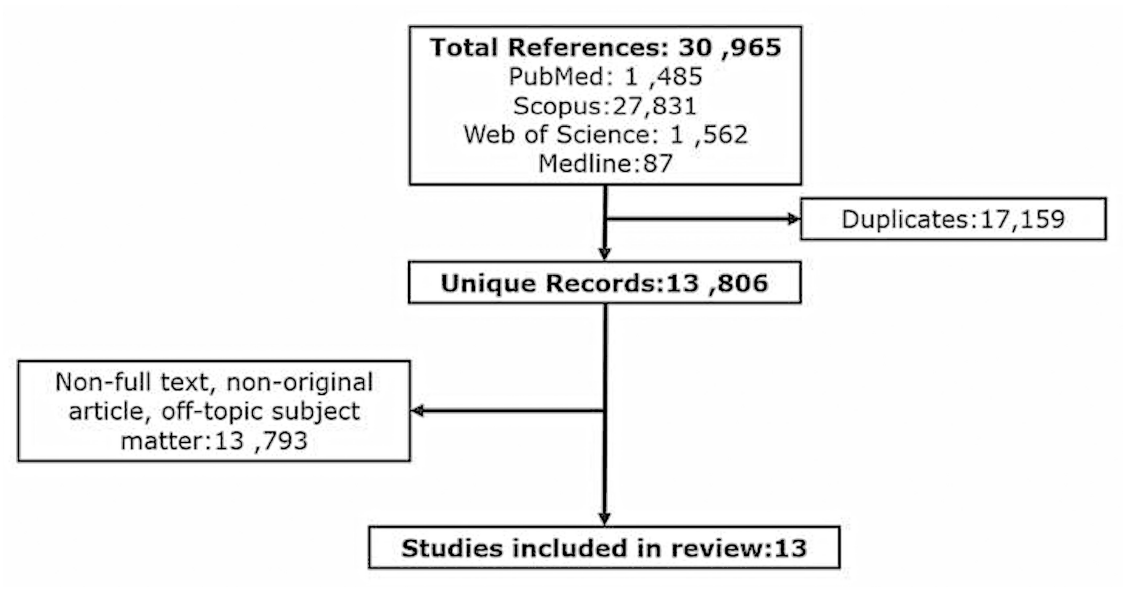

FIGURE 2 | Flow diagram showing the methodical identification and selection of studies investigating the therapeutic potential of EVs to in-vivo models of perinatal brain injury. 
TABLE 1 | Investigations of the therapeutic potential of EVs in animal models of perinatal brain injury.

\begin{tabular}{|c|c|c|c|c|c|c|c|c|c|c|}
\hline In-vivo model & EV source & $\begin{array}{l}\text { EV isolation } \\
\text { methods }\end{array}$ & $\begin{array}{l}\text { EV } \\
\text { Characterization } \\
\text { methods }\end{array}$ & $\begin{array}{l}\text { Diameter } \\
\text { range of } \\
\text { isolated } \\
\text { EVs }\end{array}$ & $\begin{array}{l}\text { Markers } \\
\text { investigated in } \\
\text { EVs }\end{array}$ & $\begin{array}{l}\text { Route, dose, and timing of } \\
\text { administration }\end{array}$ & $\begin{array}{l}\text { End time } \\
\text { points }\end{array}$ & $\begin{array}{l}\text { End point } \\
\text { measurements }\end{array}$ & Study outcomes & References \\
\hline $\begin{array}{l}\text { P9 or P10 } \\
\text { C57BL/6 mouse } \\
\text { model of HI treated } \\
\text { with either IP or } \\
\text { ipsilateral injection } \\
\text { of EVs }\end{array}$ & $\begin{array}{l}\text { Human } \\
\text { umbilical } \\
\text { cord derived } \\
\text { MSC } \\
\text { conditioned } \\
\text { media }\end{array}$ & Ultracentrifugation & $\begin{array}{l}\text {-Nanoparticle } \\
\text { tracking analysis } \\
\text {-Western blot } \\
\text {-TEM }\end{array}$ & $\begin{array}{l}30- \\
100 \mathrm{~nm}\end{array}$ & $\begin{array}{l}\text { Membrane } \\
\text { markers: CD63+ } \\
\text { Endosomal } \\
\text { markers: } \\
\text { ALIX+ } \\
\text { Tsg101+ } \\
\text { Cellular makers: } \\
\text { GM130- }\end{array}$ & $\begin{array}{l}\text { IP injection } 14 \mathrm{~h} \text { before } \mathrm{HI} \text {, } \\
\text { then immediately before } \\
\text { exposure to hypoxia, then } \\
\text { immediately after removal of } \\
\text { mice from the hypoxic } \\
\text { chamber, and finally } 3 \mathrm{~h} \text { post } \\
\text { hypoxia } \\
\text { Or } \\
2 \times 10^{5} \text { cell equivalents of } \\
\mathrm{PKH}-26 \text { stained EVs } \\
\text { transplanted into the } \\
\text { ipsilateral hemisphere } \\
(1 \mu \mathrm{L} \text { time) during } \mathrm{HI} \text { or } 6 \mathrm{~h} \\
\text { post } \mathrm{HI}\end{array}$ & $\begin{array}{l}\text { During } \mathrm{HI} \text { or } 6 \mathrm{~h} \\
\text { post } \mathrm{HI}\end{array}$ & $\begin{array}{l}\text { Histology, edema, } \\
\text { TTC, behavioral test } \\
\text { (neurological severity } \\
\text { score), distribution of } \\
\text { infrared labeled EVs }\end{array}$ & $\begin{array}{l}\text { 1. MSC-EVs reduce edema and } \\
\text { cerebral infarction volume } \\
\text { associated with HI } \\
\text { 2. MSC-EVs can significantly } \\
\text { reduce the neurological severity } \\
\text { score } \\
\text { 3. MSC-EVs help to preserve } \\
\text { the structure of neurons at the } \\
\text { microscopic level }\end{array}$ & Han et al., 2020 \\
\hline $\begin{array}{l}\text { P9 C57BL/6 } \\
\text { mouse model of HI } \\
\text { treated with IP } \\
\text { injection of EVs }\end{array}$ & $\begin{array}{l}\text { Human } \\
\text { bone } \\
\text { marrow- } \\
\text { derived } \\
\text { MSC } \\
\text { conditioned } \\
\text { media }\end{array}$ & $\begin{array}{l}\text { PEG precipitation } \\
\text { followed by } \\
\text { ultracentrifugation }\end{array}$ & $\begin{array}{l}\text {-Nanoparticle } \\
\text { tracking analysis } \\
\text {-Western blot } \\
\text {-TEM }\end{array}$ & $\begin{array}{l}108- \\
133 \mathrm{~nm}\end{array}$ & $\begin{array}{l}\text { Endosomal } \\
\text { markers: } \\
\text { Syntenin+ } \\
\text { Membrane } \\
\text { markers: CD81+, } \\
\text { CD9+, CD63+ } \\
\text { Cellular markers: } \\
\text { calnexin- and } \\
\text { prohibitin- }\end{array}$ & $\begin{array}{l}\text { IP injection of } 1 \times 10^{5} \text { cell } \\
\text { equivalents/g bodyweight } 1-\text {, } \\
3-, \text { and } 5 \text {-days post HI }\end{array}$ & 7 days $\mathrm{HI}$ & $\begin{array}{l}\text { Regional } \\
\text { neuropathological } \\
\text { scoring, atrophy, } \\
\text { histology, RT-PCR }\end{array}$ & $\begin{array}{l}\text { 1. MSC-EVs mediate an } \\
\text { anti-inflammatory response } \\
\text { 2. MSC-EVs stimulate a } \\
\text { regenerative response in } \\
\text { neurons } \\
\text { 3. MSC-EVs stimulate } \\
\text { oligodendrocyte maturation }\end{array}$ & $\begin{array}{l}\text { Kaminski et al., } \\
2020\end{array}$ \\
\hline $\begin{array}{l}\text { P9 Rice-Vannucci } \\
\text { C57/B16 mouse } \\
\text { model of HI treated } \\
\text { with intranasal } \\
\text { administration of } \\
\text { EVs }\end{array}$ & $\begin{array}{l}\text { Human } \\
\text { bone } \\
\text { marrow- } \\
\text { derived } \\
\text { MSC } \\
\text { conditioned } \\
\text { media }\end{array}$ & Ultracentrifugation & $\begin{array}{l}\text {-Nanoparticle } \\
\text { tracking analysis } \\
\text {-Electron } \\
\text { microscopy } \\
\text {-FACS } \\
\text {-Western blot }\end{array}$ & $\begin{array}{l}30- \\
1000 \mathrm{~nm}\end{array}$ & $\begin{array}{l}\text { Membrane } \\
\text { markers: CD63+ } \\
\text { CD81+ }\end{array}$ & $\begin{array}{l}\text { Intranasal administration of } \\
6 \mathrm{uL}\left(1.25 \times 10^{9}\right. \\
\text { particles/dose }) \mathrm{EV} \text { s } 1 \text { h post } \\
\text { HI }\end{array}$ & $\begin{array}{l}\text { Day } 2 \text { post EVs } \\
\text { treatment }\end{array}$ & $\begin{array}{l}\text { Histology, TUNEL } \\
\text { assay, behavioral test } \\
\text { (negative geotaxis) }\end{array}$ & $\begin{array}{l}\text { 1. MSC-EVs reduced HI } \\
\text { induced activation of aMB2 } \\
\text { microglia } \\
\text { 2. MSC-EVs reduce HI } \\
\text { mediated brain volume loss } \\
\text { 3. MSC-EVs decreased cell } \\
\text { death and improved behavioral } \\
\text { outcomes at day } 2 \text { post EV } \\
\text { treatment }\end{array}$ & Sisa et al., 2019 \\
\hline $\begin{array}{l}\text { P7 Rice-Vannucci } \\
\text { C57/B16 mouse } \\
\text { model of HI treated } \\
\text { with IC injection of } \\
\text { EVs }\end{array}$ & $\begin{array}{l}\text { Mouse bone } \\
\text { marrow- } \\
\text { derived } \\
\text { MSC (either } \\
\text { in the } \\
\text { presence or } \\
\text { absence of } \\
\text { miR-21a } \\
\text { inhibitor or } \\
\text { negative } \\
\text { control) } \\
\text { conditioned } \\
\text { media }\end{array}$ & $\begin{array}{l}\text { Ultracentrifugation } \\
\text { followed by size } \\
\text { exclusion } \\
\text { chromatography } \\
\text { (qEV column) }\end{array}$ & $\begin{array}{l}\text {-qNano Gold } \\
\text { TRPS } \\
\text {-Western blot } \\
\text {-TEM }\end{array}$ & $\begin{array}{l}60- \\
160 \mathrm{~nm}\end{array}$ & $\begin{array}{l}\text { Membrane } \\
\text { markers: CD63+ } \\
\text { CD81+ } \\
\text { CD9+ } \\
\text { Endosomal } \\
\text { markers: Tsg101+ } \\
\text { Cellular markers: } \\
\text { calnexin- }\end{array}$ & $\begin{array}{l}\text { IC injection of } 100 \text { ug } \\
\text { PKH67-labeled EVs } 1 \text { day } \\
\text { post HI } \\
\text { Or } \\
\text { Intracardial IC injection of } \\
100 \mathrm{ug} / \mathrm{mL} \text { of EVs } 1 \text { day post } \\
\text { HI }\end{array}$ & $\begin{array}{l}\text { Day } 3,5,14,21 \\
35,36,37,38, \\
39,40 \text { post } \mathrm{HI}\end{array}$ & $\begin{array}{l}\text { Histology, western } \\
\text { blot, brain water } \\
\text { content, Nissl, TTC, } \\
\text { TUNEL, FISH, TEM, } \\
\text { behavioral tests } \\
\text { (Morris water maze, } \\
\text { Y maze, hindlimb } \\
\text { suspension, cliff } \\
\text { aversion test) }\end{array}$ & $\begin{array}{l}\text { 1. MSC-EVs attenuated acute } \\
\text { brain damage and } \\
\text { neuroinflammation } \\
\text { 2. MSC-EVs stimulated } \\
\text { anti-inflammatory populations of } \\
\text { microglia and macrophages } \\
\text { 3. MSC-EVs reduce neuronal } \\
\text { apoptosis } \\
\text { 4. MSC-EVs improved injury } \\
\text { outcomes in pups } \\
\text { 5. MSC-EVs had no effect on } \\
\text { long-term memory impairment } \\
\text { 6. MSC-EVs miR-21a-5p and } \\
\text { TIMP3 cargo are essential for } \\
\text { neuroprotective effects }\end{array}$ & Xin et al., 2020 \\
\hline
\end{tabular}




\begin{tabular}{|c|c|c|c|c|c|c|c|c|c|c|}
\hline In-vivo model & EV source & $\begin{array}{l}\text { EV isolation } \\
\text { methods }\end{array}$ & $\begin{array}{l}\text { EV } \\
\text { Characterization } \\
\text { methods }\end{array}$ & $\begin{array}{l}\text { Diameter } \\
\text { range of } \\
\text { isolated } \\
\text { EVs }\end{array}$ & $\begin{array}{l}\text { Markers } \\
\text { investigated in } \\
\text { EVs }\end{array}$ & $\begin{array}{l}\text { Route, dose, and timing of } \\
\text { administration }\end{array}$ & $\begin{array}{l}\text { End time } \\
\text { points }\end{array}$ & $\begin{array}{l}\text { End point } \\
\text { measurements }\end{array}$ & Study outcomes & References \\
\hline $\begin{array}{l}\text { P7 Rice-Vannucci } \\
\text { C57/B16 mouse } \\
\text { model of HI treated } \\
\text { with IC injection of } \\
\text { EVs }\end{array}$ & $\begin{array}{l}\text { Mouse bone } \\
\text { marrow- } \\
\text { derived } \\
\text { MSC (either } \\
\text { untreated or } \\
\text { pretreated } \\
\text { with } \\
\text { hydrogen } \\
\text { sulfide and } \\
\text { in the } \\
\text { presence or } \\
\text { absence of } \\
\text { miR-7b } \\
\text { inhibitor or } \\
\text { negative } \\
\text { controls) } \\
\text { conditioned } \\
\text { media }\end{array}$ & $\begin{array}{l}\text { Ultracentrifugation } \\
\text { followed by size } \\
\text { exclusion } \\
\text { chromatography } \\
\text { (qEV column) }\end{array}$ & $\begin{array}{l}\text {-qNano Gold } \\
\text { TRPS } \\
\text {-Western blot } \\
\text {-TEM }\end{array}$ & $\begin{array}{l}60- \\
380 \mathrm{~nm}\end{array}$ & $\begin{array}{l}\text { Membrane } \\
\text { markers: } \\
\text { CD9+ } \\
\text { Endosomal } \\
\text { markers: Tsg101+ } \\
\text { Cellular markers: } \\
\text { calnexin- }\end{array}$ & $\begin{array}{l}\text { IC injection of } 100 \text { ug of EVs } \\
1 \text { day post } \mathrm{HI}\end{array}$ & $\begin{array}{l}\text { Day } 3,35,36 \text {, } \\
37,38,39 \text {, and } \\
40,42 \text { post HI }\end{array}$ & $\begin{array}{l}\text { Western blot, } \\
\text { RT-PCR, FACS, } \\
\text { TUNEL, Histology } \\
\text { TTC, Nissl, edema, } \\
\text { FISH, behavioral test } \\
\text { (Morris water maze } \\
\text { test, novel object } \\
\text { recognition test) }\end{array}$ & $\begin{array}{l}\text { 1. MSC-EVs (derived from } \\
\text { treated or untreated MSC) were } \\
\text { present in the ipsilateral } \\
\text { hemisphere } 2 \mathrm{~h} \text { post EV } \\
\text { treatment and entered both } \\
\text { microglia and neurons. } \\
\text { 2. H2S-EVs were better at } \\
\text { preventing brain tissue loss than } \\
\text { untreated EVs } \\
\text { 3. H2S-EVs promoted a more } \\
\text { anti-inflammatory brain } \\
\text { environment } \\
\text { 4. H2S preconditioning of EVs } \\
\text { was associated with improved } \\
\text { long-term cognitive and } \\
\text { memory outcomes } \\
\text { 5. H2S treatment of MSCs } \\
\text { enriches EVs with miR-7b-5p } \\
\text { and potentially are responsible } \\
\text { for effects observed. }\end{array}$ & Chu et al., 2020 \\
\hline $\begin{array}{l}\text { P7 Sprague } \\
\text { Dawley rat model } \\
\text { of HI treated with } \\
\text { IP injection of EVs }\end{array}$ & $\begin{array}{l}\text { Rat cerebral } \\
\text { cortical } \\
\text { astrocyte- } \\
\text { derived } \\
\text { conditioned } \\
\text { media }\end{array}$ & $\begin{array}{c}\text { Discontinuous } \\
\text { sucrose density } \\
\text { gradient combined } \\
\quad \text { with } \\
\text { ultracentrifugation }\end{array}$ & $\begin{array}{l}\text {-Nanoparticle } \\
\text { tracking analysis } \\
\text {-Western blot } \\
\text {-TEM }\end{array}$ & $\begin{array}{l}110- \\
132 \mathrm{~nm}\end{array}$ & $\begin{array}{l}\text { Membrane } \\
\text { markers: CD63+ } \\
\text { CD81+ } \\
\text { Endosomal } \\
\text { markers: Tsg101+ }\end{array}$ & $\begin{array}{l}\text { IP injection of } 2-3 \mu \mathrm{g} \text { of EVs } \\
24 \mathrm{~h} \text { prior to } \mathrm{HI}\end{array}$ & $\begin{array}{l}\text { Day } 1,2,3 \text {, and } \\
7 \text { post HI }\end{array}$ & $\begin{array}{l}\text { Histology, western } \\
\text { blot, TTC, TUNEL, } \\
\text { ELISA, RT-PCR, } \\
\text { oxidative stress, } \\
\text { behavioral tests } \\
\text { (righting reflex, } \\
\text { negative geotaxis } \\
\text { reflex, forepaw grip } \\
\text { test) }\end{array}$ & $\begin{array}{l}\text { 1. Astrocyte-EVs prevent HI } \\
\text { brain damage } \\
\text { 2. Astrocyte-EVs reduces } \\
\text { oxidative stress } \\
\text { 3. Astrocyte-EVs carry } \\
\text { miR-17-5p cargo that may } \\
\text { mediate effects observed. }\end{array}$ & Du et al., 2021 \\
\hline $\begin{array}{l}\text { P4 } \\
\text { Sprague-Dawley } \\
\text { rat model of IVH } \\
\text { treated with ICV } \\
\text { injection of EVs }\end{array}$ & $\begin{array}{l}\text { Human } \\
\text { umbilical } \\
\text { cord blood- } \\
\text { derived } \\
\text { MSC } \\
\text { conditioned } \\
\text { media }\end{array}$ & Ultracentrifugation & $\begin{array}{l}\text {-Nanoparticle } \\
\text { tracking analysis } \\
\text {-Western blot } \\
\text {-TEM } \\
\text {-SEM }\end{array}$ & $\begin{array}{l}50- \\
100 \mathrm{~nm}\end{array}$ & $\begin{array}{l}\text { Membrane } \\
\text { markers: CD63+ } \\
\text { CD81+ } \\
\text { CD9+ } \\
\text { Cellular markers: } \\
\text { Cytochrome C-, } \\
\text { Fibrillarin-, GM130- }\end{array}$ & $\begin{array}{l}\text { ICV injection of } 20 \text { ug of EVs } \\
\text { two days post IVH }\end{array}$ & $\begin{array}{c}\text { Rat pups } \\
\text { monitored daily } \\
\text { for the first } \\
7 \text { days post IVH. } \\
\text { Subsequent } \\
\text { monitoring } \\
\text { weekly until P32. } \\
\text { MRI at day } 7 \text { and } \\
\text { day } 28 \text { post IVH. }\end{array}$ & $\begin{array}{l}\text {-Histology, MRI, } \\
\text { ELISA, behavioral } \\
\text { test (negative } \\
\text { geotaxis and rotarod } \\
\text { test) }\end{array}$ & $\begin{array}{l}\text { 1. MSC-EVs performed similarly } \\
\text { to MSCs in preventing IVH } \\
\text { induced brain injuries, } \\
\text { progression of } \\
\text { post-hemorrhagic } \\
\text { hydrocephalus, and improved } \\
\text { behavioral outcomes } \\
\text { 2. MSC-EVs mediate } \\
\text { neuroprotective activities } \\
\text { through BDNF signaling }\end{array}$ & Ahn et al., 2021 \\
\hline $\begin{array}{l}\text { P3 Wistar rat } \\
\text { model of LPS/HI } \\
\text { treated with IP } \\
\text { injection of EVs }\end{array}$ & $\begin{array}{l}\text { Human } \\
\text { bone } \\
\text { marrow- } \\
\text { derived } \\
\text { MSC } \\
\text { conditioned } \\
\text { media }\end{array}$ & $\begin{array}{l}\text { PEG precipitation } \\
\text { followed by } \\
\text { ultracentrifugation }\end{array}$ & $\begin{array}{l}\text {-Nanoparticle } \\
\text { tracking analysis } \\
\text {-Western blot }\end{array}$ & $\begin{array}{l}\text { Detail not } \\
\text { provided }\end{array}$ & $\begin{array}{l}\text { Endosomal } \\
\text { markers: Tsg101+ } \\
\text { Membrane } \\
\text { markers: } \\
\text { CD81+ } \\
\text { Microbial: negative } \\
\text { for } \\
\text { bacteria and } \\
\text { viruses }\end{array}$ & $\begin{array}{l}\text { Two repetitive IP injections of } \\
\text { EVs }\left(1 \times 10^{8} \text { cell }\right. \\
\text { equivalents } / \mathrm{kg}) 3 \mathrm{~h} \text { prior to } \\
\text { and } 24 \mathrm{~h} \text { after IP injection of } \\
\text { the vehicle or LPS }\end{array}$ & $\begin{array}{c}\text { Day } 2,8,27,87, \\
122 \text { post EVs } \\
\text { treatment }\end{array}$ & $\begin{array}{l}\text { Histology, western } \\
\text { blotting, TUNEL } \\
\text { assay, cytokine } \\
\text { analysis, RT-PCR, } \\
\text { diffusion tensor-MRI, } \\
\text { behavioral tests } \\
\text { (Barnes maze, novel } \\
\text { object recognition } \\
\text { test, open field test) }\end{array}$ & $\begin{array}{l}\text { 1. MSC-EVs reduce } \\
\text { inflammation related neuronal } \\
\text { degeneration and microgliosis } \\
\text { 2. MSC-EVS prevent reactive } \\
\text { astrogliosis } \\
\text { 3. MSC-EVs prevented } \\
\text { myelination defects and white } \\
\text { matter damage } \\
\text { 4. MSC-EVs treated rats have } \\
\text { improved long-term cognitive } \\
\text { function compared to untreated } \\
\text { controls }\end{array}$ & $\begin{array}{l}\text { Drommelschmidt } \\
\text { et al., } 2017\end{array}$ \\
\hline
\end{tabular}


TABLE 1 | (Continued)

\begin{tabular}{|c|c|c|c|c|c|c|c|c|c|c|}
\hline In-vivo model & EV source & $\begin{array}{l}\text { EV isolation } \\
\text { methods }\end{array}$ & $\begin{array}{l}\text { EV } \\
\text { Characterization } \\
\text { methods }\end{array}$ & $\begin{array}{l}\text { Diameter } \\
\text { range of } \\
\text { isolated } \\
\text { EVs }\end{array}$ & $\begin{array}{l}\text { Markers } \\
\text { investigated in } \\
\text { EVs }\end{array}$ & $\begin{array}{l}\text { Route, dose, and timing of } \\
\text { administration }\end{array}$ & $\begin{array}{l}\text { End time } \\
\text { points }\end{array}$ & $\begin{array}{l}\text { End point } \\
\text { measurements }\end{array}$ & Study outcomes & References \\
\hline $\begin{array}{l}\text { P3 Sprague } \\
\text { Dawley rat model } \\
\text { of white matter } \\
\text { damage (method } \\
\text { unclear) treated } \\
\text { with ICV injection } \\
\text { of EVs }\end{array}$ & $\begin{array}{l}\text { Rat bone } \\
\text { marrow- } \\
\text { derived } \\
\text { MSC } \\
\text { conditioned } \\
\text { media }\end{array}$ & Ultracentrifugation & $\begin{array}{l}\text {-TEM } \\
\text {-Western blot }\end{array}$ & 60-100 nm & $\begin{array}{l}\text { Membrane } \\
\text { markers: CD63+ } \\
\text { Cellular markers: } \\
\text { calnexin- }\end{array}$ & $\begin{array}{l}\text { ICV EV injection. Dosage and } \\
\text { timing of injection in relation } \\
\text { to induction of injury unclear. }\end{array}$ & $\begin{array}{l}\text { Days } 1,3,5 \text {, and } \\
7 \text { post- EVs } \\
\text { treatment }\end{array}$ & Histology, ELISA & $\begin{array}{l}\text { 1. MSC-EVs enhanced } \\
\text { secretion of protective factors } \\
\text { 2. MSC-EVs reduced secretion } \\
\text { of pro-inflammatory factors } \\
\text { 3. MSC-EVs improved } \\
\text { prognosis of brain injury }\end{array}$ & Shu et al., 2018 \\
\hline $\begin{array}{l}\text { P3 Wister rat } \\
\text { model of LPS/HI } \\
\text { treated with } \\
\text { intranasal } \\
\text { administration of } \\
\text { EVs }\end{array}$ & $\begin{array}{l}\text { Human } \\
\text { Wharton's } \\
\text { jelly umbilical } \\
\text { cord MSC- } \\
\text { derived } \\
\text { conditioned } \\
\text { media }\end{array}$ & Ultracentrifugation & $\begin{array}{l}\text {-TEM } \\
\text {-Exo-Check } \\
\text { Exosome Antibody } \\
\text { array }\end{array}$ & $16-87 \mathrm{~nm}$ & $\begin{array}{l}\text { Membrane } \\
\text { markers: CD63+ } \\
\text { CD81+ } \\
\text { Flotilin-1+ } \\
\text { EpCam+ } \\
\text { ICAM+ } \\
\text { Endosomal } \\
\text { markers: } \\
\text { TSG101+ } \\
\text { ANXA5+ } \\
\text { ALIX+ } \\
\text { Cellular makers: } \\
\text { GM130- }\end{array}$ & $\begin{array}{l}\text { Intranasal administration of } \\
50 \mathrm{mg} / \mathrm{kg} \text { of EVs } \\
\text { administered at the time of IP } \\
\text { LPS injection } \\
\text { OR } \\
\text { Intranasal administration of } \\
10 \mathrm{mg} / \mathrm{kg} \mathrm{IRDye}{ }^{\circledR} \\
800 \mathrm{CW} \text {-labeled EVs } \\
\text { administered at the time of IP } \\
\text { LPS injection }\end{array}$ & $\begin{array}{c}30 \text { min, } 3 \text { h, day } \\
1 \text { and } 8 \text {, and } \\
4 \text {-weeks post } \\
\text { LPS/HI }\end{array}$ & $\begin{array}{l}\text { Histology, western } \\
\text { blot, RT-PCR, } \\
\text { TUNEL assay, } \\
\text { distribution of } \\
\text { infrared labeled EVs, } \\
\text { behavioral tests } \\
\text { (Morris water maze) }\end{array}$ & $\begin{array}{l}\text { 1. MSC-EVs were detected in } \\
\text { frontal region of brain } 30 \text { min } \\
\text { post intranasal administration } \\
\text { 2. MSC-EVs distribute } \\
\text { throughout brain at } 3 \mathrm{~h} \\
\text { post-administration } \\
\text { 3. Neuron specific cell death is } \\
\text { reduced with EV administration } \\
\text { prior to ischemia } \\
\text { 4. Numbers of oligodendrocytes } \\
\text { and neurons were restored in } \\
\text { groups treated with MSC-EVs } \\
\text { 5. MSC-EV treatment improved } \\
\text { learning outcomes }\end{array}$ & $\begin{array}{l}\text { Thomi et al., } \\
\text { 2019a }\end{array}$ \\
\hline $\begin{array}{l}\text { P3 Wister rat } \\
\text { model of LPS/HI } \\
\text { treated with } \\
\text { intranasal } \\
\text { administration of } \\
\text { EVs }\end{array}$ & $\begin{array}{l}\text { Human } \\
\text { Wharton's } \\
\text { jelly umbilical } \\
\text { cord MSC- } \\
\text { derived } \\
\text { conditioned } \\
\text { media }\end{array}$ & Ultracentrifugation & $\begin{array}{l}\text {-TEM } \\
\text {-Exo-Check } \\
\text { Exosome Antibody } \\
\text { array }\end{array}$ & $16-87 \mathrm{~nm}$ & $\begin{array}{l}\text { Membrane } \\
\text { markers: CD63+ } \\
\text { CD81+ } \\
\text { Flotilin-1+ } \\
\text { EpCam+ } \\
\text { ICAM+ } \\
\text { Endosomal } \\
\text { markers: } \\
\text { TSG101+ } \\
\text { ANXA5+ } \\
\text { ALIX+ } \\
\text { Cellular makers: } \\
\text { GM130- }\end{array}$ & $\begin{array}{l}\text { Intranasal administration of } \\
50 \mathrm{mg} / \mathrm{kg} \text { of EVs } \\
\text { administered at time of IP } \\
\text { injection of LPS }\end{array}$ & $\begin{array}{l}\text { Day } 1 \text { post EVs } \\
\text { treatment }\end{array}$ & $\begin{array}{l}\text { Histology, RT-PCR, } \\
\text { ELISA }\end{array}$ & $\begin{array}{l}\text { 1. MSC-EVs were detected in } \\
\text { brain } \\
\text { 2. MSC-EVs reduce microglia } \\
\text { mediated neuroinflammation }\end{array}$ & $\begin{array}{l}\text { Thomi et al., } \\
2019 \mathrm{~b}\end{array}$ \\
\hline $\begin{array}{l}\text { GA d106 fetal } \\
\text { sheep model of HI } \\
\text { treated with IV } \\
\text { injections of EVs }\end{array}$ & $\begin{array}{l}\text { Human } \\
\text { bone } \\
\text { marrow- } \\
\text { derived } \\
\text { MSC } \\
\text { conditioned } \\
\text { media }\end{array}$ & $\begin{array}{l}\text { PEG followed by } \\
\text { low-speed } \\
\text { centrifugation }\end{array}$ & $\begin{array}{l}\text {-Nanoparticle } \\
\text { tracking analysis } \\
\text {-Western blot }\end{array}$ & $\begin{array}{l}\text { 99-123 nm } \\
\text { (ZetaView) } \\
\text { and 133-- } \\
138 \mathrm{~nm} \\
\text { (NanoSight) } \\
\end{array}$ & $\begin{array}{l}\text { Membrane } \\
\text { markers: } \\
\text { CD81+ } \\
\text { Endosomal } \\
\text { markers: } \\
\text { TSG101+ } \\
\text { Microbial: negative } \\
\text { for bacteria, } \\
\text { viruses and } \\
\text { endotoxins }\end{array}$ & $\begin{array}{l}\text { IV doses of EVs }\left(2.0 \times 10^{7}\right. \\
\text { cell equivalents) administered } \\
1 \mathrm{~h} \text { and } 4 \text { days post } \mathrm{HI}\end{array}$ & $\begin{array}{l}\text { Day } 1 \text { before } \mathrm{HI}, \\
\text { Day } 1,2,3,4,5, \\
6 \text {, and } 7 \text { post } \mathrm{HI}\end{array}$ & $\begin{array}{l}\text { Histology, } \\
\text { electrophysiological } \\
\text { brain parameters }\end{array}$ & $\begin{array}{l}\text { 1. MSC-EV treatment reduced } \\
\text { number and duration of } \\
\text { seizures indicating improved } \\
\text { cortical function. } \\
\text { 2. MSC-EV treatment preserved } \\
\text { baroreceptor reflex indicating } \\
\text { improved brain stem function } \\
\text { 3. MSC-EV treatment showed a } \\
\text { trend only toward protection } \\
\text { against hypomyelination (not } \\
\text { statistically significant) }\end{array}$ & $\begin{array}{l}\text { Ophelders et al., } \\
\quad 2016\end{array}$ \\
\hline $\begin{array}{l}\text { GA d106 fetal } \\
\text { sheep model of HI } \\
\text { treated with IV } \\
\text { injections of EVs }\end{array}$ & $\begin{array}{l}\text { Human } \\
\text { bone } \\
\text { marrow- } \\
\text { derived } \\
\text { MSC } \\
\text { conditioned } \\
\text { media }\end{array}$ & $\begin{array}{l}\text { PEG followed by } \\
\text { low-speed } \\
\text { centrifugation }\end{array}$ & $\begin{array}{l}\text {-Nanoparticle } \\
\text { tracking analysis } \\
\text {-Western blot } \\
\text {-TRPS }\end{array}$ & $\begin{array}{l}\text { Detail not } \\
\text { provided }\end{array}$ & $\begin{array}{l}\text { Membrane protein } \\
\text { markers: } \\
\text { CD81+ } \\
\text { Endosomal protein } \\
\text { markers: } \\
\text { TSG101+ }\end{array}$ & $\begin{array}{l}\text { IV doses of EVs }\left(2.0 \times 10^{7}\right. \\
\text { cell equivalents) administered } \\
1 \mathrm{~h} \text { and } 4 \text { days post } \mathrm{HI}\end{array}$ & $\begin{array}{c}\text { Day } 1,3 \text {, and } 7 \\
\text { post } \mathrm{HI}\end{array}$ & $\begin{array}{l}\text { Histology, western } \\
\text { blot }\end{array}$ & $\begin{array}{l}\text { 1. MSC-EVs expressing } \\
\text { Annexin A1 prevented albumin } \\
\text { leakage into the brain indicating } \\
\text { maintenance of BBB integrity in } \\
\text { injured animals }\end{array}$ & $\begin{array}{l}\text { Gussenhoven } \\
\text { et al., } 2019\end{array}$ \\
\hline
\end{tabular}




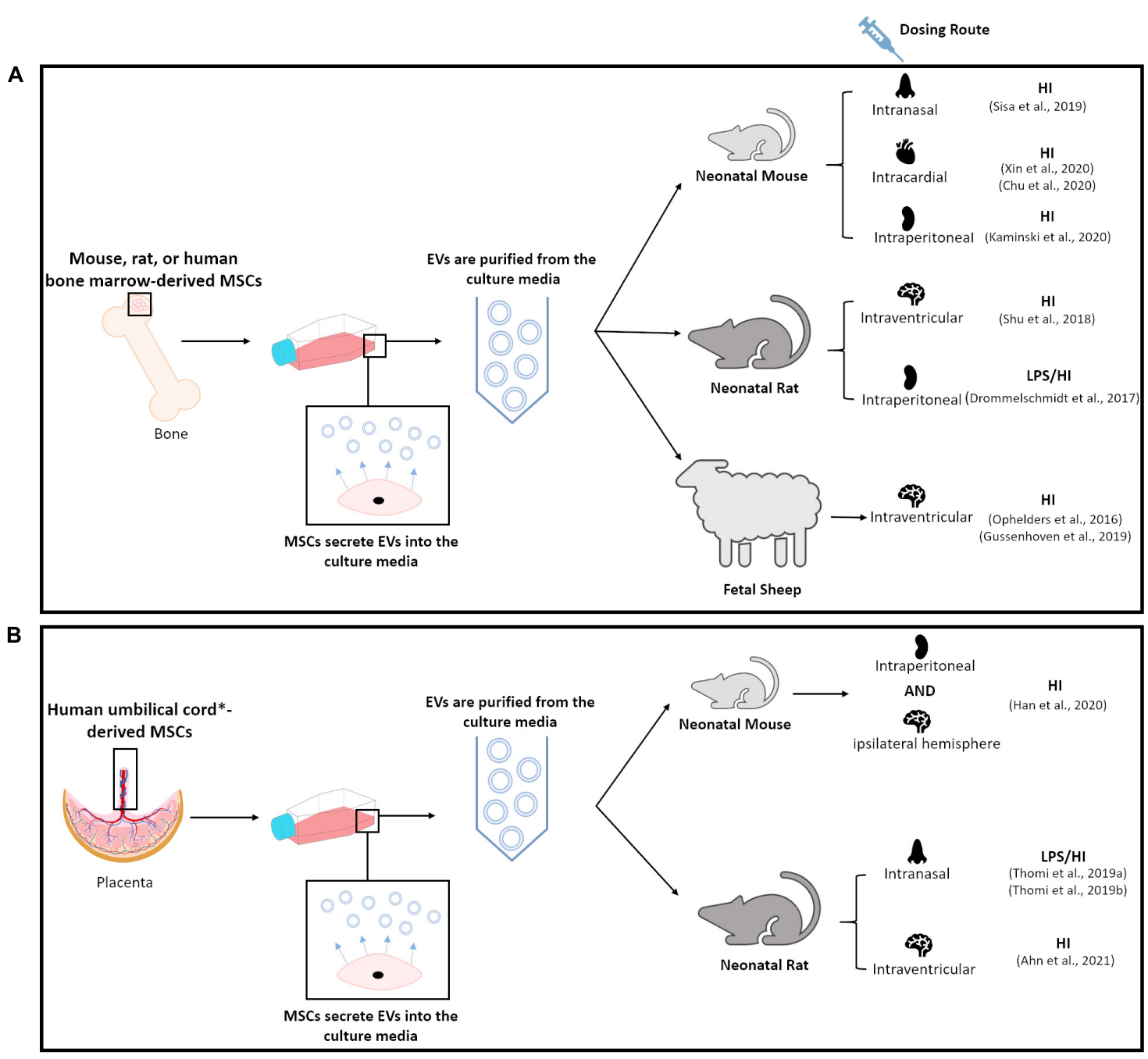

c

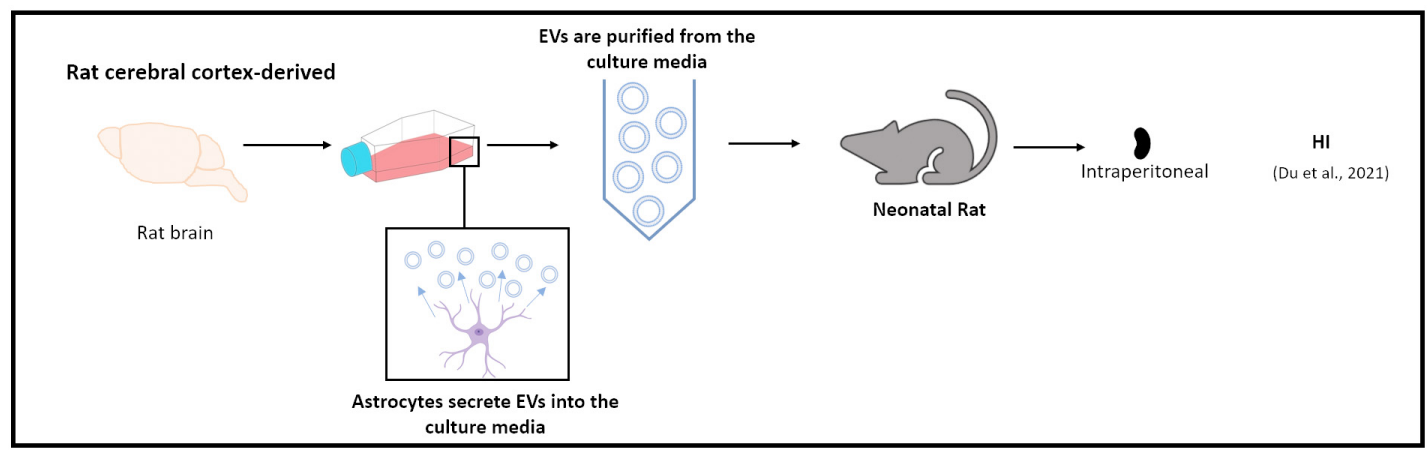

FIGURE 3 | Schematic diagram summarizing methodologies utilized in publications identified by this unbiased review of literature. (A) Highlights studies that utilized bone marrow-derived MSCs. (B) Highlights studies that utilized umbilical cord*, umbilical cord blood* or umbilical cord Wharton's jelly derived MSCs. (C) Highlights study which utilized rat cortical-derived astrocytes.

and intercellular adhesion molecules (ICAM)] and endosomal markers [Tumor susceptibility gene 101 (TSG101), Annexin A5 (ANXA5), and ALG-2-interacting protein X (ALIX)], and stained negatively for cellular markers (GM130, a cis-Golgi apparatus marker, which monitors cellular contamination of EV isolations, calnexin, an endoplasmic reticulum stress marker, prohibitin and cytochrome $\mathrm{C}$, mitochondrial markers, and fibrillarin, a nucleolar protein) as well as markers of bacteria, viruses and endotoxins (Table 1).

Besides consideration of the optimal source of EVs, the most efficient route of $\mathrm{EV}$ administration is also a matter of debate. While intravenous (IV) administration is convenient, it 
can lead to a high accumulation of EVs in peripheral organs, such as the lung, liver, and spleen (Somiya et al., 2017). However, intranasal administration of therapeutics bypasses peripheral elimination and directly targets the CNS via neural and olfactory pathways innervating the nasal cavity (Merkus and van den Berg, 2007; Hanson and Frey, 2008; Pardridge, 2012). Moreover, researchers consider intranasal administration less invasive and more efficient compared to IV, intracranial, and intra-arterial routes of administration, thus long-term intranasal administration would provide optimal therapeutic application of EVs. However, only $3 / 13$ of the perinatal studies identified in this systemic review have utilized intranasal administration of EVs in their animal models (Figure 3; Sisa et al., 2019; Thomi et al., 2019a,b). Despite the intranasal route offering several advantages, the authors of the other studies identified in this review adopted either intraperitoneal (IP) (Drommelschmidt et al., 2017; Han et al., 2020; Kaminski et al., 2020; Du et al., 2021), IV (Ophelders et al., 2016; Gussenhoven et al., 2019), intracardial (IC) (Chu et al., 2020; Xin et al., 2020), intracerebroventricular (ICV) (Shu et al., 2018; Ahn et al., 2021), or ipsilateral hemisphere (Han et al., 2020) routes of administration of EVs (Figure 3).

Like the mode of delivery, the dosage and duration of delivery also varied between studies. Furthermore, some studies administered EV therapy as a single dose post-injury (Sisa et al., 2019; Thomi et al., 2019b; Chu et al., 2020; Xin et al., 2020; Ahn et al., 2021), whereas others as a repeated dose either prior to and/or following injury (Ophelders et al., 2016; Drommelschmidt et al., 2017; Gussenhoven et al., 2019; Han et al., 2020; Kaminski et al., 2020; Du et al., 2021). There were also differences in the normalization methods of dose; it was either based on a $\mathrm{mg} / \mathrm{kg}$ body weight (Thomi et al., 2019b), cell equivalents/kg body weight (Ophelders et al., 2016; Drommelschmidt et al., 2017; Gussenhoven et al., 2019; Han et al., 2020; Kaminski et al., 2020), particles/dose (Sisa et al., 2019) or $\mu \mathrm{g}$ of protein (Chu et al., 2020; Xin et al., 2020; Ahn et al., 2021; Du et al., 2021) approach (Table 1). In addition, one study did not provide clear information of the timing and dosage regimen of EVs (Shu et al., 2018).

Clinical presentation of injuries, as well as timing of the initial onset is often unclear and difficult to determine (Ophelders et al., 2020). This is especially apparent in the case of HI injuries, since observed latency for first appearance of seizures can vary from minutes to days following birth (Naeye and Lin, 2001). Despite this limitation, more work is required to determine the most efficacious therapeutic window for initiating EV therapy, since it is more likely that therapeutic interventions delivered, as close to the initial onset of injury, will have a greater chance of enhancing positive long-term neurological outcomes. Equally important, is the need to determine whether time-windows for intervention of EVs can be lengthened.

\section{EV Therapy Attenuates Microscopic and Macroscopic Damage}

Preterm infants, with prior evidence of focal and diffuse white matter lesions, invariably display atypical cortical and subcortical development, involving volumetric reductions in both total cerebral gray and myelinated white matter and larger ventricular volumes either at term or later in childhood (Inder et al., 1999, 2005; Miller et al., 2003). Furthermore, prematurity is frequently associated with a reduction to the size of the corpus callosum, the largest white matter tract within the brain (Schmahmann and Pandya, 2006), which is considered to contribute to later adverse neurodevelopmental outcomes (Caldú et al., 2006). In this context, several preclinical models of perinatal brain injury have shown beneficial effects of EVs in relation to injuryinduced deficits in regional volumes and ventricular size of the immature brain.

Based on histological analysis of the Nissl-pattern of staining, intranasal administration of human bone marrow MSC-EVs in a neonatal rat model of $\mathrm{HI}$, significantly reduced mean ipsilateral volume deficits in the pyriform cortex (by 35\%), thalamus (by $12.91 \%$ ), and external capsule (by 10.83\%) compared to untreated animals (Sisa et al., 2019). Similarly, neuropathological assessment in cresyl violet stained sections of the neonatal mouse brains collected 7 days after $\mathrm{HI}$ and following repeated (1, 3, and 5 days after HI) bolus IP injections of human bone marrow MSC-EVs demonstrated a significant decrease in tissue atrophy within the striatum region compared to both platelet-derived EV (control EVs) and untreated HI controls (Kaminski et al., 2020). Additionally, studies of repeated IP injections of umbilical cord MSC-EVs to neonatal mice both $14 \mathrm{~h}$ before $\mathrm{HI}$ and immediately before and $3 \mathrm{~h}$ following $\mathrm{HI}$, have revealed a significant reduction in edema and cerebral infarction volume compared to untreated HI mice (Han et al., 2020). Further, histological examination of neonatal mice brains $72 \mathrm{~h}$ following HI revealed systemic administration of mouse bone marrow MSC-EVs $24 \mathrm{~h}$ following HI, attenuated both edema and infarction volume of the ipsilateral hemisphere, as well as cortical tissue loss compared to untreated animals (Xin et al., 2020). However, in some representative coronal sections there was no effect of EV treatment on infarction volume and tissue loss of the hippocampus (Xin et al., 2020). Using the same neonatal mouse model, the authors later tested hydrogen sulfide pretreated MSC-EVs; hydrogen sulfide enriches secreted EVs with miR-7b-5p cargo, an miRNA that has been previously shown to decrease during the first 1-3 days of reperfusion following transient cerebral ischemia in adult rodents (Dharap et al., 2009). Results showed that hydrogen sulfide preconditioning of MSC-EVs, further enhanced their therapeutic ability to reduce cerebral hemispheric infarct volumes and edema (Chu et al., 2020). Moreover, IP administration of rat astrocyte-derived EVs to $\mathrm{P} 7 \mathrm{HI}$ rats similarly reduced infarct volume and also decreased neuronal cell death (TUNEL ${ }^{+}$cells) compared to untreated HI controls (Du et al., 2021).

Studies conducted by Shu et al. (2018) in which bone marrow MSC-EVs were injected into the lateral ventricle of neonatal rats, hematoxylin and eosin staining showed an absence of cellular edema within areas of the white matter surrounding the lateral ventricle and reduced ventricular enlargement. However, the absence of relevant information as to the nature of the cerebral insult imposed and very limited histopathological assessment performed is a major limitation to this study. Interestingly, Ahn et al. (2021) observed a comparable reduction in ventricular 
enlargement using serial MRI at P11 and P32 using an IVH rat model treated with human umbilical cord blood-derived MSCEVs compared to untreated IVH controls. Notably, this effect was not observed in the fibroblast-derived EV controls suggesting this neuroprotective action is specific to MSC-EVs (Ahn et al., 2021).

At the microscopic level, hypomyelination is a central feature of preterm brain injury, which principally affects preoligodendrocytes (or late oligodendroglia progenitors), that predominate in the forebrain at 24-32 weeks gestation (Back et al., 2001; Volpe et al., 2011; Buser et al., 2012; van Tilborg et al., 2016). In addition, such myelination disturbances are considered to arise through depletion of preoligodendrocytes, because of cell death or their subsequent inability to fully differentiate into mature myelinating oligodendrocytes (Back et al., 2001; Volpe et al., 2011; Buser et al., 2012; van Tilborg et al., 2016). Consequently, researchers have sought to investigate the impact that administration of EVs may have in protecting preoligodendrocytes and in turn whether they are able to ameliorate myelination deficits.

Ophelders et al. (2016) investigated the potential of EV therapy to reduce myelination deficits in preterm fetal sheep subjected to umbilical cord occlusion. Fetal systemic administration of human bone marrow MSC-EVs were performed; two boluses of $2.0 \times 10^{7}$ cell equivalents were injected $1 \mathrm{~h}$ and 4 days postumbilical cord occlusion and fetuses killed 7 days thereafter (Ophelders et al., 2016). Treatment improved electrocortical function (namely a decrease in the number and duration of seizures) and a preservation of baroreceptor reflex was associated with partial improvement in myelination as indicated by an increase in the intensity of myelin basic protein (MBP; a marker of mature oligodendrocytes) staining in the subcortical white matter regions of the brain (Ophelders et al., 2016). However, this effect was not paralleled by a reduction in overall cell death, since assessment of apoptotic cell death within subcortical white matter regions revealed no effect of IV EV therapy (Ophelders et al., 2016).

Similarly, in a P2 neonatal rat model of combined inflammation/HI-induced brain injury, intranasal administration of MSC-EVs had no effect on immature and mature oligodendrocyte-specific cell death $\left(\mathrm{TUNEL}^{+} \mathrm{Olig}^{+}\right.$ cells) within regions of the corpus callosum following brain injury (Thomi et al., 2019b). However, whilst untreated or vehicle treated neonatal rat models of perinatal brain injury have impaired myelination compared to healthy animals (reduced expression of MBP by 41 and $81 \%$ at the gene and protein levels, respectively) (Drommelschmidt et al., 2017; Thomi et al., 2019b), small EV treatment either ameliorated or partially restored myelination (increased expression of MBP by 17 and $38 \%$ at the gene and protein levels) following injury (Drommelschmidt et al., 2017; Thomi et al., 2019b). Moreover, long-term follow-up of microstructural white matter changes provided further supportive evidence of the beneficial effect of EVs. Diffusion tensor MRI (DT-MRI) assessment at P125 of P2 rats subjected to LPS/HI-induced brain injury revealed that in EV-treated animals there was increased fractional anisotropy accompanied by reduced radial diffusivity supporting restoration of the microstructure of the corpus callosum (Drommelschmidt et al., 2017). In addition, in a P4 rat model of IVH induced by injection of maternal blood to both lateral ventricles of the brain, ICV administration of MSC-EVs significantly improved myelination 32 days after injury (Ahn et al., 2021). Furthermore, the effect was specific to MSC-EVs since treatment with control fibroblast-EVs was without an effect.

Studies undertaken in the neonatal mouse model of $\mathrm{HI}$ also demonstrates that while the number of oligodendrocytes is not impacted by bone marrow-derived MSC-EV treatment, there is enhanced oligodendrocyte maturation and myelination specifically within the striatum and white matter of the external capsule (Kaminski et al., 2020). Of note, treatment with MSCEVs results in a decrease in the number of the immature $\mathrm{O}^{+}$oligodendrocytes and an increase in both the number of $\mathrm{CC}^{+}{ }^{+}$differentiated oligodendrocytes and the expression of MBP (Kaminski et al., 2020). Accordingly, this data suggests that MSCEVs can adversely impact oligodendrocyte differentiation rather than cell numbers.

Like oligodendrocyte precursors, subplate neurons are also selectively vulnerable to degeneration in the preterm brain (Kinney et al., 2012; Back and Miller, 2014; Schneider and Miller, 2019). Intranasal MSC-EV therapy in the P2 neonatal rat model of preterm brain injury, evoked by combined HI/LPS, was recently shown to significantly reduce neuronal cell death (TUNEL ${ }^{+} \mathrm{NeuN}^{+}$cells) in the subplate zone of the posterior parietal cortex as well as the CA1 region of the hippocampus (Thomi et al., 2019b). EV therapy also significantly increased both gene and protein expression of microtubule-associated protein 2 (MAP2, a dendritically enriched protein and a marker of synaptic plasticity) in the ipsilateral hemisphere by 19 and 33\%, respectively, following injury (Thomi et al., 2019b). Similarly, in the P4 IVH neonatal rat model, $\mathrm{TUNEL}^{+}$cell death was reduced and neurogenesis enhanced upon treatment with MSCEVs (Ahn et al., 2021). Additionally, therapeutic administration of EVs significantly reduced cell death in the external capsule, cortex and striatum in a mouse model of perinatal bran injury (Sisa et al., 2019; Chu et al., 2020; Kaminski et al., 2020; Xin et al., 2020). Moreover, MSC-EV treatment ameliorated HIinduced ultrastructural characteristics of neuronal cell damage (discontinuous double membrane structures, vacant cytoplasm, swollen mitochondria and reduced nuclear chromatin) (Han et al., 2020; Xin et al., 2020) and improved neural organization (Han et al., 2020). Collectively, the current literature suggests the use of MSC-EVs in preclinical animal models is an attractive alternative to cell-based therapies to restore myelination and neuronal deficits following perinatal brain injury.

\section{EV Therapy Helps Maintain the Integrity of the BBB}

During early human development, the $\mathrm{BBB}$, which protects the brain, was previously viewed as being incomplete. However, evidence has emerged indicating that tight junctional proteins such as claudin-5, occludin, and junctional adhesion molecule (JAM)-1, which are all critical for maintaining the integrity of the $\mathrm{BBB}$ are expressed in the germinal matrix, cortex, and white matter of the fetal human brain as early as 16 weeks GA 
(Ballabh et al., 2005). This suggests that the BBB is functionally developed relatively early in GA, ensuring healthy development of the fetal brain.

Insults to the brain, including $\mathrm{HI}$ can lead to a reduction in both the density of blood vessels and a reduction in the functionality of the BBB. For example, in the mouse model of perinatal brain injury there is a significant reduction in vessel density in the striatum compared to sham operated animals (Kaminski et al., 2020). Interestingly this reduction in vessel density is correlated to a reduction in the number of proliferative $\mathrm{CD} 1^{+}$endothelial cells (Kaminski et al., 2020). However, treatment with human bone marrow MSC-EVs leads to increased vessel density, particularly within the neurogenic subventricular zone as a result of enhanced proliferation of $\mathrm{CD} 31^{+}$endothelial cells (Kaminski et al., 2020).

An additional complication encountered following insults to the brain is the release of free radicals that can cause disruption of the fetal $\mathrm{BBB}$ by altering tight junctional molecules (Kumar et al., 2008; Lee et al., 2017). In near-term fetal sheep, free radicals released in response to $\mathrm{HI}$ and reperfusion injury can alter expression of tight junctional proteins such as claudin-5, occludin, zonal occludin-1 and zonal occludin-2 (Chen et al., 2012). Furthermore, in preterm fetal sheep, quantification of BBB permeability by assessment of the immunohistochemical distribution of endogenous albumin has revealed that HIinduced leakage of the $\mathrm{BBB}$ at day 7 following injury is ameliorated by prior IV administration of two boluses of MSCEVs $\left(2 \times 10^{7}\right.$ cell equivalents $)$ at $1 \mathrm{~h}$ and 4 days following injury (Gussenhoven et al., 2019). Although permeability was not assessed by a fluorescence-based approach, such as IV infusion of FITC-labeled albumin, results suggested that MSC-EV treatment might help to maintain BBB integrity. Additionally, treatment of sham control fetal sheep with MSC-EVs did not incur albumin leakage suggesting that systemic administration of MSC-EVs is a safe therapeutic strategy (Gussenhoven et al., 2019).

\section{Immunoregulatory and Antioxidant Effects of EV Therapy}

A common inflammatory response accompanying the acute degeneration of preoligodendrocytes in non-cystic and diffuse white matter injury is reactive astrocytosis and activated microgliosis (Burda and Sofroniew, 2014). Upon activation, astrocytes and microglia produce a wide array of inflammatory molecules that can have either harmful or beneficial consequences (Hagberg et al., 1996; Kadhim et al., 2001; Baburamani et al., 2014; McNamara and Miron, 2020).

Mesenchymal stem cell-derived EVs are known to have multifaceted immunomodulatory properties and can notably ameliorate inflammatory and apoptotic processes through suppression of pro-inflammatory cytokine production (Ma et al., 2014; Gomzikova et al., 2019), therefore therapeutic administration of MSC-EVs to the injured developing brain may regulate gliosis and other pro-inflammatory mediators. Indeed, in the studies identified by this unbiased review, seven rodent models of perinatal brain injury report a reduction in populations of both astrocytes and microglia in areas of the brain, namely the corpus callosum, cortex, hippocampus and striatum following treatment with MSC-EVs (Drommelschmidt et al., 2017; Sisa et al., 2019; Thomi et al., 2019b; Chu et al., 2020; Kaminski et al., 2020; Xin et al., 2020; Ahn et al., 2021). Of these seven studies, two were conducted in a P3 neonatal rat model of inflammation-induced brain injury reproduced by IP injection of LPS. Combined results from these studies demonstrated that either IP (Drommelschmidt et al., 2017) or intranasal (Thomi et al., 2019b) administration of MSC-EVs attenuated microgliosis (within regions of the corpus callosum, cingulate white matter, and internal capsule) and prevented astrogliosis (within regions of cingulate white matter and internal capsule). Furthermore, attenuation of gliosis was directly associated with a reduction in inflammatory-induced hypomyelination (Drommelschmidt et al., 2017), which is in accordance with evidence of chronic diffuse reactive gliosis within damaged cerebral white matter regions of infants born prematurely (Buser et al., 2012; Back, 2014). Furthermore, findings reported by Drommelschmidt et al. (2017) strengthen the hypothesis that EVs are likely to have no adverse effects, especially since this study underwent a repeated dosing regimen, involving administration of EVs $3 \mathrm{~h}$ before and $24 \mathrm{~h}$ following LPS injection. These same authors were able to show comparable numbers of astrocytes and microglia within the cortex and white matter between sham-control and sham-control+MSC-EV treated P3 neonatal rats. Similarly, Ahn et al. (2021) demonstrated in a P4 neonatal rat model of IVH, ICV administration of umbilical cord blood-derived MSC-EVs significantly reduced the number of $\mathrm{GFAP}^{+}$astrocytes and ED1positive microglia, 32 days after injury.

Furthermore, four studies were undertaken in either a P8 (Chu et al., 2020; Xin et al., 2020) or P9 (Sisa et al., 2019; Kaminski et al., 2020) mouse model of term HI, which exhibits predominately gray matter damage (Vannucci and Vannucci, 1997). MSCEV administration in these studies reduced the numbers of both infiltrating macrophages and brain-resident microglia (Chu et al., 2020; Xin et al., 2020). Specific regional assessment of microglial activation revealed a significant reduction within the cortex, hippocampus and striatum following intranasal, IC, or IP treatment with MSC-EVs (Sisa et al., 2019; Chu et al., 2020; Kaminski et al., 2020; Xin et al., 2020). In addition, MSCEV administration also appeared to reverse the "ameboid-like" appearance of ionized calcium binding adaptor molecule 1 (Iba1) stained microglia and skewed the ratio of pro-inflammatory M1 macrophages/anti-inflammatory M2 macrophages toward a more anti-inflammatory state compared to untreated $\mathrm{HI}$ animals (Xin et al., 2020). It is worth noting that the reduction in microglia within the brains of $\mathrm{HI}$ animals was further enhanced through the use of hydrogen sulfide pretreated MSC-EVs with statistically significant differences between untreated MSC-EVs and hydrogen sulfide pretreated MSC-EVs (Chu et al., 2020). IP administration of MSC-EVs appears not to impact the expression of the M1-cell surface marker, CD86, or the M2-cell surface marker, CD206, on microglia post-insult (Kaminski et al., 2020). However, it does reduce the A1-type reactive astrocytic expression of complement C3 protein, which is characteristically upregulated following HI (Hammad et al., 2018; Kaminski et al., 2020) and recognized as having harmful pro-inflammatory and 
neurotoxic effects (Hammad et al., 2018). Furthermore, it does not impact A2-type reactive astrocytic expression of pentraxin 3 (PTX3), which is developmentally regulated and associated with beneficial anti-inflammatory and pro-regenerative effects (Kaminski et al., 2020).

The above findings are largely in line with those observed by Ophelders et al. (2016) in preterm fetal sheep. They report similar levels of microglial immunoreactivity within subcortical white matter and hippocampal regions between sham-control and sham-control+MSC-EVs fetuses. In the same study, however, MSC-EV treatment failed to mitigate HI-induced microglial activation within the hippocampus. Of concern, MSC-EV treatment exacerbated $\mathrm{HI}$-induced microglial activation within the subcortical white matter, suggesting the neuroprotective mechanism of EV therapy may not involve modulation of inflammatory cells. Interestingly though, splenic weight relative to body weight, which is indicative of activation of a splenic inflammatory response, thus activation of peripheral immune system, was significantly reduced following $\mathrm{HI}$ and was restored with MSC-EV administration. However, removal of the spleen also reduces stroke-induced neurodegeneration in an adult rat model, suggesting abatement of the splenic pro-inflammatory response is key to reducing inflammatory-induced cerebral injury (Ajmo et al., 2008; Seifert et al., 2012). Whether the discrepancies observed in modulation of gliosis between these various animal models are a consequence of both the route and timing of EV administration and/or timing of assessment of histopathological outcomes post-insult is unclear.

Aside from the cellular changes observed in response to EV treatment, researchers utilizing rodent models have also examined changes to levels of pro-inflammatory, proapoptotic, and neuroprotective markers during the early stages postinjury. Thomi et al. (2019b) found that at the protein level, MSC-EVs decreased production of pro-inflammatory cytokines $\mathrm{TNF} \alpha$ (by $43.6 \%$ ) and IL- $1 \beta$ (by $42 \%$ ) in P3 neonatal brain lysates collected $24 \mathrm{~h}$ after LPS/HI-induced injury. Presumably, MSC-EVs effects on microgliosis, as noted previously in this study, would have contributed to the down-regulation of proinflammatory cytokines. In addition, gene expression profiles of brain lysates revealed MSC-EVs suppressed LPS-induced upregulation of chemokine (C-X-C motif) ligand-10 (Cxcl10), IL$1 \beta$, IL- 8 and TNF $\alpha$ transcripts. It is worth mentioning also that in the same study, co-culturing of BV-2 microglial cells with MSCEVs interfered with TLR4/CD14 signaling pathway, suggesting a mechanism by which EVs mitigate pro-inflammatory production (Thomi et al., 2019b).

In a similar study, Drommelschmidt et al. (2017) observed no effect of MSC-EVs on levels of TNF $\alpha$ and IL-18 mRNA or protein expression in the brain or serum, $48 \mathrm{~h}$ after LPS injection. The lack of effect of EVs noted in this study may relate to acute inflammatory responses already being resolved within the $48 \mathrm{~h}$ time frame post-LPS injection; however, it could equally reflect other differences such as dosing regimens, routes, and data collection points (Table 1). This is also in contrast to findings reported by Chu et al. (2020) in a mouse model of term HI, where EV therapy attenuated HI-induced mRNA expression of proinflammatory cytokines, CD11b, CD32, CD86, cyclooxygenase
(COX2), IL-1 $\beta$, IL-6, inducible nitric oxide synthase (iNOS), and $\mathrm{TNF} \alpha$; an effect which was further enhanced through the use of hydrogen sulfide pretreated MSC-EVs. Similar observations were made by Ahn et al. (2021) in a P4 neonatal rat model of IVH. In their study, both CSF and periventricular brain tissue levels of pro-inflammatory cytokines, IL- $1 \alpha$, IL- $1 \beta$, IL- 6 and TNF $\alpha$, were significantly reduced following treatment with MSC-EVs compared to both fibroblast-derived EV controls and untreated IVH controls.

Both antenatal and neonatal exposure to endotoxins, such as bacterial LPS, are well documented to trigger innate immunity in the CNS, resulting in microglial activation and oligodendrocyte progenitor and neuronal cell apoptosis in both white and gray matter (Wang et al., 2006). Administration of MSC-EVs significantly reduces apoptosis (caspase-3 protein expression) and cellular degeneration (TUNEL ${ }^{+}$cells) in both the cortex and white matter in P3 neonatal rats $48 \mathrm{~h}$ post LPS injection (24 h post second dose of EVs) (Drommelschmidt et al., 2017). Furthermore, Shu et al. (2018) reported findings to suggest that EVs induce an increase in TGF $\beta$ protein expression in association with reduced brain injury. Since anti-apoptotic and anti-excitotoxic effects of TGF $\beta$ are well documented, this may represent a mechanism by which EVs reduce the neurodegenerative cascade driven by inflammatory responses. There is conclusive evidence that neuroprotective responses to insults, such as $\mathrm{HI}$, can involve up-regulation of TGF $\beta$, which serves to ameliorate the extent of injury. Notably, however, there are also reports of TGF $\beta$ being neurotoxic (Wyss-Coray et al., 1995; Manaenko et al., 2014).

Intuitively, approaches to reduce both oxidative stress and pro-inflammatory damage would be a desirable feature of therapies. IP administration of astrocyte-derived EVs immediately prior to $\mathrm{HI}$, in $\mathrm{P} 7$ rats, can effectively reduce brain tissue protein levels of TNF- $\alpha$ and IL- $1 \beta$ and those of oxidative stress factors, SOD, CAT, glutathione peroxidase (GPX) and malondialdehyde (MDA) (Du et al., 2021). Thus, at least, astrocyte-derived EVs appear to have the capacity to promote a neuroprotective environment by way of suppression of oxidative stress- and pro-inflammatory-induced damage.

Taken together, the data reviewed is supportive of the proven anti-inflammatory and anti-apoptotic properties of MSC-EVs (Cho et al., 2019b; Wiklander et al., 2019), and the observed antiinflammatory and anti-oxidant effects of astrocyte-EVs (Du et al., 2021) are likely to be an underlying principal mechanism that helps to reduce perinatal brain injury.

\section{EV Therapy Improves Behavioral Functional Outcome}

Seven studies have investigated whether EVs repress behavioral impairments associated with perinatal brain injury. Four were conducted in mouse models of term HI (Sisa et al., 2019; Chu et al., 2020; Han et al., 2020; Xin et al., 2020) and four were undertaken in neonatal rat models of preterm HI (Du et al., 2021), LPS/HI (Drommelschmidt et al., 2017; Thomi et al., 2019b) and IVH (Ahn et al., 2021) injury. 
Interestingly, in the study where repeated IP injections were conducted of umbilical cord MSC-EVs to neonatal mice $14 \mathrm{~h}$ before, immediately before, and $3 \mathrm{~h}$ following $\mathrm{HI}$, a significant decline in neurological severity scores for neurological assessment of movement, reflex, sensation, and balance is observed $6 \mathrm{~h}$ following HI (Han et al., 2020). Furthermore, in the P9 mouse model of term $\mathrm{HI}$, behavioral negative geotaxis tests conducted at P11, $48 \mathrm{~h}$ after $\mathrm{HI}$ confirmed that EV treated animals display a significant decrease in the time required to rotate $180^{\circ}$ to face uphill after release compared to HI untreated animals (Sisa et al., 2019). Similarly, in the P7 model of term HI, hindlimb suspension tests conducted at P10 and P12 demonstrated that MSC-EV treatment attenuates hindlimb impairment observed in untreated HI animals (Xin et al., 2020). In addition, MSC-EV treatment also reduced the latency to complete the cliff avoidance test suggesting that MSC-EV treatment improves both depth perception and visual impairment post-HI compared to untreated animals (Xin et al., 2020). Moreover, mice treated with MSC-EVs also perform better in the Y-maze test for spatial learning and memory (Xin et al., 2020). Moreover, while untreated HI animals spent less time in the novel arm of the maze compared to sham operated animals, MSC-EV treated HI animals spent more time on the novel arm (Xin et al., 2020). Strikingly, while EV-treated HI animals performed similarly to their untreated counterparts in the novel object recognition test and escape latency tests, $\mathrm{HI}$ animals treated with hydrogen sulfide pretreated MSC-EVs had an enhanced performance in both tests (Chu et al., 2020). Finally, MSC-EV treated HI mice also exhibited improved, albeit insignificant, performance during the Morris Water Maze test for long-term memory compared to untreated HI mice (Xin et al., 2020). Their performance was comparable to that of hydrogen sulfide pretreated MSC-EV treated animals (Chu et al., 2020). While the data from the mouse models appears promising, evidence from the four neonatal rat studies is varied.

The study by Drommelschmidt and colleagues was the only study to provide a comprehensive longitudinal follow-up assessment of long-term behavioral outcomes (Drommelschmidt et al., 2017). The authors, however, did not observe any effect of either EV treatment or LPS/HI on learning behavior or motor activity and anxiety-related behavior as assessed by Barnes Water Maze and Open Field tests. Nevertheless, they did suggest that since animals underwent every behavioral test at P30 (adolescent) and P90 (adult), it could have confounded the results (Drommelschmidt et al., 2017). Notably, however, 30 and 90 days after EV administration, they did show an improvement in adaptive memory (Barnes Water Maze test; latency to find the escape hole), and non-spatial and nonaversive memory functions (Novel Object Recognition test; time exploring novel object) compared to animals exposed to LPS/HI alone (Drommelschmidt et al., 2017). Their MRI finding of restoration of white matter microstructure at P125, as previously discussed, agrees well with such improvements in behavioral function following EV treatment.

In later studies, Thomi et al. (2019b) confirmed that in P2 rats, intranasal administration of MSC-EVs following LPS exposure and prior to $\mathrm{HI}$, significantly improved spatial learning performance 4 weeks after brain injury compared to untreated injured animals, as assessed by Morris Water Maze test. Although there was no treatment benefit shown in terms of either short-term or long-term memory performance, their findings of improved myelination together with reduced neuronal cell death in the parietal cortex and hippocampus correlates well with the observed improvements in learning performance (Thomi et al., 2019b).

More recently, Ahn et al. (2021) reported improved behavioral functions following MSC-EV treatment of IVH neonatal rats. They were able to show improvements in balance, coordination, physical condition, and motor-planning using the negative geotaxis (described previously) and the rotarod test, whereby animals were tasked with fall avoidance from a rotating suspended rod. Specifically, they noted the time taken for rats to re-orientate themselves in the negative geotaxis test was shortened when rats were treated with MSC-EVs. These times were comparable to MSC cell treatment of IVH animals, but were notably decreased compared to fibroblast-EV treated animals or untreated IVH controls. The MSC-EV treated animals showed a significant increase (like that of MSC treated animals) in the time spent on the rotating rod compared to fibroblast-EV treated animals or untreated IVH controls.

Finally, neurobehavioral outcomes were also assessed in the neonatal rat model treated IP with rat astrocyte-derived EVs (Du et al., 2021). Assessments were made at 1, 3, and 7 days following $\mathrm{HI}$ using the negative geotaxis test (described above), the righting reflex test, where animals are removed from their normal upright position and timed for the reflex which allows them to correctly orientate themselves for brain recovery assessment, and the forepaw grip test, which assesses force and fatigability. Compared to untreated HI control rats, a reduction in both the negative geotaxis response and the righting reflex was observed in rats treated with astrocyte-EVs compared to untreated HI controls. Moreover, forepaw grip was increased in astrocyte-EV treated animals compared to untreated HI controls.

In summary, behavioral studies in rodent models of perinatal brain injury demonstrate that EV treatment not only can improve early neurological deficit scores associated with HI, but also longterm changes in performance of motor coordination (Negative Geotaxis and Rotarod test), spatial learning (Morris Water Maze), adaptive memory (Barnes Water Maze) and non-spatial and nonaversive memory (Novel Object Recognition). Collectively, while this suggests MSC-EVs and astrocyte-EVs have benefits in terms of improving neurobehavioral outcome, more in-depth analyses are needed to resolve contradictory findings that exist between these various studies.

\section{EV Therapy Improves Electrophysiological Brain Function}

Larger animal models, such as the fetal sheep model have the advantage of being able to provide measurements of clinically relevant endpoints for the therapeutic application of EVs against perinatal brain injury that would not otherwise be possible in rodent models. The larger size of fetal sheep facilitates continuous measurement of several physiological 
variables including electrophysiological brain activity by electroencephalogram (EEG), electrophysiological cardiac function by electrocardiogram (ECG) and hemodynamics by blood pressure and heart rate. In fetal sheep, prolonged umbilical cord occlusion is associated with long-lasting suppression of parasagittal cortical EEG activity, with delayed onset of seizures (Williams et al., 1990; Reddy et al., 1998; Fraser et al., 2005; Lee et al., 2009). In human preterm and term infants, these events predictably correlate with adverse neurological outcomes (Inder et al., 2003b; Murray et al., 2009).

As previously discussed, Ophelders et al. (2016) are presently the only researchers to have conducted a study investigating the therapeutic potential of EVs by assessing electrophysiological brain function in fetal sheep. Although they observed only a modest trend toward protection against HI-induced myelination with IV MSC-EV therapy, which was in contrast to their previous therapeutic study utilizing IV MSC therapy (Jellema et al., 2013b), they showed improved electrocortical function; namely a decrease in the number and duration of seizures. This is consistent with their previous finding demonstrating IV MSC treatment after HI induction reduced seizure activity (Jellema et al., 2013b). An assessment of baroreflex sensitivity (i.e., baroreflex-mediated heart rate) was also undertaken, since dysregulation or impairment of baroreflex function is postulated to augment existing cerebral damage following $\mathrm{HI}$ in reperfusion phase (Zwanenburg et al., 2013). IV administration of MSC-EVs to fetuses after $\mathrm{HI}$ induction prevented $\mathrm{HI}$-induced impairment of baroreflex reflex sensitivity 3-6 days following HI (Ophelders et al., 2016). However, sham-control MSC-EV treated fetuses displayed impaired baroreflex sensitivity to levels, which was lower than that of fetuses who were subjected to HI alone. This raises real concerns as it may indicate IV administered MSCEVs may limit the efficacy of the baroreflex-mediated heart rate response to buffer changes in blood pressure. Given the above, further investigations are required to understand these physiological outcomes reported with EV therapy and whether they translate to long-term improvement of cognitive and motor function. Equally important is the need to determine whether differences in dose and timing of administration may alter or further enhance observed beneficial outcomes.

\section{Candidate Molecular Mediators of EV Therapeutic Activity}

While studies identified in this review of the literature suggest MSC-EVs are a useful therapeutic candidate in the treatment of perinatal brain injury, most do not provide a potential mechanism of action through which MSC-EVs exert their effects. There are five exceptions to this, as discussed in detail below.

Two of these papers highlight candidate proteins (Gussenhoven et al., 2019; Ahn et al., 2021). Firstly, the study by Gussenhoven et al. (2019), showing treatment with MSC-EVs helps to maintain BBB integrity in a fetal sheep model of preterm brain injury. They hypothesized that Annexin A1 (an essential endogenous regulator of $\mathrm{BBB}$ integrity) expressed on the surface of MSC-EVs interacts with the Annexin A1/Formyl peptide receptor to maintain BBB integrity
(Gussenhoven et al., 2019). Moreover, they performed elegant invitro experiments demonstrating MSC-EVs expressing Annexin A1 or recombinant Annexin A1 improved trans-endothelial electrical resistance (a measure of $\mathrm{BBB}$ integrity) of primary fetal rat endothelial cells and that addition of formyl peptide receptor blockers mitigated the effect (Gussenhoven et al., 2019).

Secondly, Ahn et al. (2021) highlight the potential for brain derived neurotrophic factor (BDNF) carried in the cargo of umbilical cord blood-derived MSC-EVs in mediating the neuroprotective effects observed in their P4 neonatal rat model of IVH. Specifically, they demonstrated that the neuroprotective roles of MSC-EVs were attenuated by siRNA transfection of BDNF in MSC-EVs at the macroscopic, microscopic, inflammatory, and behavioral levels. An observation that was not apparent when IVH neonatal rats were treated using the MSCEVs transfected with random-sequence scrambled siRNA. That BDNF may be a critical neuroprotective mediator is unsurprising given its neuroprotective properties have been identified in a number of neuropathologies including HI (Chen et al., 2013), IVH (Ahn et al., 2017; Ko et al., 2018), meningitis (Bifrare et al., 2005), and traumatic brain injury (Wurzelmann et al., 2017). It is possible that BDNF activation of the tyrosine kinase receptor $\mathrm{B}$ ( $\mathrm{Tkr} \mathrm{B})$, which is highly expressed in the neonatal brain (Webster et al., 2006), may promote neuroprotective and neuroregenerative effects (Chen et al., 2013).

The remaining four highlight candidate miRNAs (Chu et al., 2020; Han et al., 2020; Xin et al., 2020; Du et al., 2021). Firstly, is the study conducted by Xin et al. (2020) in a mouse model of term HI, which showed MSC-EV treatment helped to reduce neuroinflammation, improve neuronal survival and enhance behavioral outcomes. This study used next generation sequencing to profile the miRNA contents of the MSC-EVs used therapeutically (Xin et al., 2020). Using this technique, miR21a-5p was identified as a potential mediator of the therapeutic effects observed (Xin et al., 2020). Through both in-vitro and in-vivo assessment it was observed that MSC-EVs enhanced expression of miR-21a-5p, which was significantly reduced in response to HI (Xin et al., 2020). Moreover, the authors proposed that miR-21a-5p may increase both the survival and proliferation of neuronal cells by targeting and reducing the activity of the proapoptotic mediator tissue inhibitor of metalloproteinase 3 (Timp3) (Xin et al., 2020).

Secondly, is the study by Chu et al. (2020), involving pretreatment of MSCs with hydrogen sulfide to generate MSCEVs enriched with miR-7b-5p. Results suggested miR-7b-5p was responsible for enhancing both the neuroprotective and antiinflammatory effects of MSC-EV s by binding to the $3^{\prime}$-UTR of the FOS gene. In addition to these candidate molecules, several other miRNAs have been proposed as potential therapeutic mediators and it is possible that they are delivered via EVs and could be investigated in EV-derived miRNA screening panels (Cho et al., 2019b). For example, miR-592 can target the prostaglandin D2 receptor (PTGDR) and the neutrophin receptor (NTR) p75, thus preventing proapoptotic signaling in neurons (Irmady et al., 2014; Sun et al., 2018). Furthermore, EV-derived miR$133 \mathrm{~b}$ can inhibit connective tissue growth factor (CTGF) and Ras homology family member A (RhoA) expression allowing 
for neurite outgrowth (Xin et al., 2012, 2013b), whereas EV encapsulated miR-124 induces neurogenesis (Yang et al., 2017).

Thirdly is the elegant study by Han et al. (2020) who identified miR-410 encapsulated within umbilical cord-derived MSC-EVs as a potential mediator of therapeutic effects using a combination of in-vitro, molecular biology, in-silico, and in-vivo techniques. Specifically, they identified that miR-410 encapsulated MSC-EVs could inhibit neuronal apoptosis in OGD primary murine neuronal cells (Han et al., 2020). They also demonstrated, using the neonatal mouse, that EV-derived miR410 reduced the neurological severity score, edema, and cerebral infarction volume compared to untreated HI animals (Han et al., 2020). Moreover, Han et al. (2020) demonstrated using both in-silico investigation and RNA expression that miR-410 may mediate its effects by down-regulating the expression of histone deactelayse 1 (HDAC1), which in turn enhances the expression of early growth response 2 (EGR2)/B-cell lymphoma (Bcl2) and prevents apoptosis.

Finally the fourth study, conducted by Du et al. (2021), demonstrated astrocyte-EV encapsulated miR-17-5p cargo reduced neuronal apoptosis and inflammation in the HI neonatal rat. Their study demonstrated an inverse relationship exists between miR-17-5p carried by astrocyte EVs and expression of the proapoptotic protein BCL2 Interacting Protein 2 (BNIP2) in the neonatal rat brain. Specifically, treatment using astrocyte-EV encapsulated miR-17-5p cargo resulted in a decrease in BNIP2 expression in the rat HI brain, while depletion of miR-17-5p cargo from astrocyte-EVs resulted in an increase in BNIP2 expression in the rat HI brain. Interestingly, an inverse relationship also existed between miR-17-5p and the proapoptotic protein BCL2 associated X, apoptosis regulator (BAX) (Wang et al., 2020). The authors also noted a decrease in BAX in the brains of rats treated with astrocyte-EVs. Hence, it is likely that the miR-17-5p cargo of astrocyte-EVs down-regulates both BAX and BNIP resulting in an increase in anti-apoptotic $\mathrm{Bcl} 2$ expression, which potentially contributes in part to the neuroprotective effects observed in this study.

It is possible that other molecular mediators that can confer neuroprotective and neuroregenerative properties are packaged as EV cargo. However, future work is required to identify these molecules, their mechanisms of action, whether they are cell of origin specific, and whether they preferentially target specific cell types to exert their effects.

\section{Reported Complications and Side-Effects}

The results of the current literature are promising in terms of the therapeutic use of EVs in preclinical animal models of perinatal brain injury. Despite this, the administration of any therapeutic agent can bring with it complications and side effects. The administration of EVs appears well tolerated in the rat model and deleterious side effects, such as weight loss, death or clinical illness have not been reported (Drommelschmidt et al., 2017). Moreover, EV treatment of rats with perinatal brain injury results in an $18 \%$ increase in survival rate compared to those not treated (Thomi et al., 2019b). It is unclear whether this is true for the mouse model.

All the studies identified in this review investigated the role of EVs in the treatment of perinatal brain injury. In doing so, the researchers tend to focus on the brain alone. Yet it is possible that therapeutic administration of EVs likely results in the biodistribution of EVs to non-targeted regions. Presently, only three perinatal studies have sought to investigate the in-vivo biodistribution of EVs after administration and their off-target outcomes (Thomi et al., 2019b; Chu et al., 2020; Xin et al., 2020).

Experiments conducted in a P2 neonatal rat model of LPS/HI brain injury revealed that infrared dye (IRDye) ${ }^{\circledR} 800 \mathrm{CW}$-labeled MSC-EVs appeared in the frontal region of the brain, including the olfactory bulb, $30 \mathrm{~min}$ after intranasal administration and were evenly distributed throughout the brain within $3 \mathrm{~h}$ (Thomi et al., 2019b). Within non-targeted regions of the body only a small portion of EVs were identified in the trachea and gastrointestinal tract at $30 \mathrm{~min}$ and $3 \mathrm{~h}$ following intranasal administration and were absent in the spleen (Thomi et al., 2019b). No adverse effects were found in relation to the observed peripheral distribution. However, a limitation of this study is that it did not provide an extensive biodistribution investigation of organs well known to accumulate EVs, such as the liver, lung, kidney and heart (Wiklander et al., 2015). Nevertheless, this study strongly supports an intranasal route of administration as a means of efficiently delivering EVs to the developing brain. In addition, Chu et al. (2020) administered DiD-labeled EVs IC into a mouse model of term $\mathrm{HI}$ and detected their presence within the brain from $2 \mathrm{~h}$ post injection. Data from the same group using PKH67-labeled EVs delivered IC, established targeting to neurons $(36.9 \%)$, astrocytes $(21.5 \%)$, and microglia $(34.3 \%)$ in the ipsilateral cortex (Xin et al., 2020). Unsurprisingly, given the method of EV delivery, PKH67-labeled EVs were found in other organs, including the liver, kidney and spleen (Chu et al., 2020; Xin et al., 2020). That both reports indicate that MSCEVs can target the brain tissue and that distribution to the spleen is either absent or greatly reduced, suggests therapeutically administered EVs may not incite an immunological reaction. Regardless, the biodistribution of therapeutically delivered EVs requires further investigation as the research in this area is minimal. Understanding the in-vivo fate of EVs is of utmost importance for future therapeutic applications.

As a whole, the findings suggest that from a therapeutic perspective MSC-EVs are well tolerated and are less likely to accumulate in peripheral organs when administered intranasally. Whether IV administration has similar outcomes of biodistribution and tolerance remains unknown and requires investigation.

\section{DISCUSSION}

The studies identified in this unbiased review investigating the potential use of EVs as a therapeutic treatment for perinatal brain injury, report an overall improvement in neurological outcomes. The studies highlight the ability of EVs to modulate inflammatory responses associated with both preterm and term acquired brain injuries. Because of these beneficial properties, 
they are successful in improving white matter and gray matter microstructural integrity through decreasing both gliosis and pro-inflammatory cytokine production and improving cell survival through amelioration of apoptosis. Additionally, EV therapy improved cognitive deficits (e.g., memory function and exploratory activity) and enhanced the performance in motor coordination function. These results are comparable to that of adult experimental models of neurological brain injury, including traumatic brain injury and stroke, where EV administration imparts neuroprotective and neuroregenerative effects (Xin et al., 2013a; Doeppner et al., 2015; Zhang et al., 2015). This suggests that with further research, EVs have the potential to be a useful therapeutic option in the treatment of perinatal brain injury.

Preclinical translation of EVs, however, is confounded by contrary publications on the effects of EVs. Contradictory results are most likely attributable to differences in animal experimental models, EV cell source, dosage of EVs, and route and timing of EV administration. This makes comparisons between studies difficult and limits interpretation of results. Although EVs represent a promising next-generation treatment for perinatal brain injury, future progress is dependent on a number of considerations. For example, while only $1 / 13$ studied utilized astrocyte-EVs, 12/13 of the studies identified in this review utilized MSC-EVs, derived from different sources; either from the umbilical cord, umbilical cord blood, Wharton's jelly of the human umbilical cord, or from adult bone marrow.

EVs isolated from MSC cultures of Wharton's jelly have the notable advantage of coming from the umbilical cord, an extra-embryonic tissue, which is normally discarded at birth and free of ethical donor concerns and relatively low in cost (Frausin et al., 2015; Donders et al., 2018). Furthermore, there is evidence to show that MSCs derived from extra/embryonic/fetal tissues are more superior than adult bone marrow as they have a greater differentiation and expansion potential (Can and Karahuseyinoglu, 2007; Batsali et al., 2013). In addition, the controversy regarding the cellular heterogeneity in MSC cultures has crossed from cell-based therapies into EV-based therapies, thus appropriate characterization of the cell source (i.e., the MSC) is critical for reproducibility of therapeutic data utilizing MSC-EVs (Mo et al., 2016; Robey, 2017).

An important consideration will be to determine if the functional effects of MSC-EVs are reproducible using EVs from different cell sources. In this regard, it will also be crucial to establish any potential differences in mechanisms mediating EV therapeutic effects, which may exist between EV cell sources and their various small and large EV subpopulations. This is relevant, since heterogeneous populations exist and current isolation methods are relatively inadequate in resolving them (Gould and Raposo, 2013; Smith et al., 2015; Taylor and Shah, 2015; Willms et al., 2016, 2018; Jeppesen et al., 2019; Burbidge et al., 2020). Moreover, such comparisons are clearly necessary when designing EV therapeutic strategies for perinatal brain injury, since the immune-suppressive potential and thus ability to ameliorate neuroinflammation, including the potential to promote neuroregeneration are of paramount importance.

Current evidence suggests EVs secreted by cells exhibit target selection and that the EV cellular source must be matched to requirements of the target application and that EVs adopt a similar homing pattern to their cell source (Marcus and Leonard, 2013; Wiklander et al., 2015). Thus, it is feasible that generation of neural stem cell-derived EVs would be more likely to exhibit the highest distribution to the developing brain and confer neuroprotective benefits. Indeed, a few studies analyzing the efficacy of neural stem cell-derived EVs have shown robust results in both adolescent and adult stroke models (Webb et al., 2018a,b; Zhang et al., 2020).

Aside from the original cell type from which EVs are isolated, the impact of cell culture parameters and microenvironment in the production of EVs, including the passage number, seeding density, cell confluence, and the frequency and time of EV collection should be taken into consideration. While considerable progress has been made in EV production methods, the passage number is of particular relevance since evidence suggests the neuroprotective effect of human umbilical cord Wharton's jelly MSCs (and their paracrine activities) diminish with increasing passage number (Bonab et al., 2006; Izadpanah et al., 2008; Patel et al., 2017; Dabrowska et al., 2018). Furthermore, reduced cell seeding density is attributable to greater production of EVs (Lieberman and Glaser, 1981; Théry et al., 2002; Patel et al., 2017). The culture conditions (e.g., oxygen concentration, media composition, $2 \mathrm{D}$ vs. $3 \mathrm{D}$ cultures) in which the source cells are cultured should be considered as these may influence not only the yield, but also the function of EVs released into the culture media (Park et al., 2018; Patel et al., 2018; Guo et al., 2019). This is particularly true given the differences Ophelders and colleagues noted in terms of the anti-inflammatory properties conveyed by MSCs and MSC-EVs following HI-induction in preterm fetal sheep (Jellema et al., 2013b; Ophelders et al., 2016). The authors postulated that such differences perhaps relate to fundamental differences in environments (Ophelders et al., 2016). Intuitively, endogenous MSCs have an innate capacity to sense their surrounding conditions and thus tailor their secreted factors (e.g., either pro- or anti-inflammatory EV cargo or other factors) to prevailing conditions. However, in the situation of invivo $\mathrm{EV}$ administration, $\mathrm{EV}$ cargo load is predetermined by the cellular response of cells to the culture environment.

Unfortunately, the lack of standardization of protocols between research groups investigating the therapeutic role of EVs in the treatment of perinatal brain injury adds a level of complexity in understanding the biochemical mechanisms at play. If future studies aim to standardise the cells from which EVs are isolated, including the methods, dosage and timing of EV administration, it may be possible to use an -omics approach to identify the mechanisms by which stem cell-derived EVs exert their functional effects. Once these biochemical mechanisms are identified we may be able to employ methods to enrich for candidate molecules in EVs as has been done in the study by Chu et al. (2020) or to bioengineer EVs containing molecules of interest to help optimize therapeutic molecule delivery.

Given the research funding environment, it is not surprising that individual research groups have chosen to focus on determining whether individual proteins (Annexin A1 and BDNF) and miRNA (miR-21a-5p, miR-7b-5p, miR-410, and miR-17-5p) are potential mediators of the neuroregenerative 
and neuroprotective effects observed in in-vivo models of $\mathrm{HI}$ treated using EVs. However, since each study utilized different $\mathrm{EV}$ isolation methods, vesicle cargo likely reflects the specific subpopulation of EVs isolated (Thery et al., 2018). Moreover, of the four studies investigating the role of miRNA EV cargo as mediators of therapeutic benefit, three (Chu et al., 2020; Han et al., 2020; Du et al., 2021) utilized enzymatic/detergent treatment. It is unclear whether the miRNA identified in the remaining study (Xin et al., 2020) was encapsulated within isolated EVs, attached to their surface of EVs, or simply contaminants co-isolated with the EV subpopulation. To help limit such ambiguity in future studies, researchers should be guided by the parameters recommended in the ISEV position papers (Mateescu et al., 2017; Thery et al., 2018) prior to the commencement of their research. By doing so will ensure optimal study design that include additional controls and detailed description of both sample processing and analysis that would enhance the overall quality and reproducibility of research publications.

Nevertheless, understanding the impact of individual mediators is critical to identifying which molecules that potentially mediate optimal neuroregenerative and neuroprotective responses from a nanotherapeutics development perspective, since synthetically manufactured EVs loaded with a specific cargo could be developed as an "off the shelf" product. However, biologically it is more likely that a combination of molecules (DNA/RNA/small RNA/protein/lipid/other) working synergistically will provide the greatest beneficial effect.

In-vivo biodistribution of EVs is determined by the route of administration (Wiklander et al., 2015, 2019). The rat study conducted by Thomi et al. (2019b) clearly demonstrates that intranasal administration of EVs is effective in targeting the brain. This route of administration is relatively non-invasive and would be easily translatable to the clinic. However, further research is required regarding the effectiveness of intranasal delivery of EVs in humans compared to animal models, especially given that the nasal olfactory mucosa (entry point to the brain) in rats covers approximately $45 \%$ more of the total nasal epithelium than in humans (Gross et al., 1982; Jiang et al., 2011).

In order to advance translation of EVs to the neonatal intensive care unit, appropriate preclinical animal models of brain injury are required to improve understanding of the underlying mechanism(s) by which EVs may exert protective effects. Such models will be crucial in establishing optimal routes and timing of administration and therapeutic effects, including any adverse effects. Furthermore, there must be appropriate negative control experiments. Surprisingly, of the perinatal studies reviewed, only two were identified where control experiments were performed using EV-depleted conditioned media (therapeutically inert EV control, isolated under identical conditions as experimental EVs) to exclude the possibility of effects through co-isolated proteins and common EV components (Shu et al., 2018; Han et al., 2020). Others utilized control EVs in the form of fibroblast-derived EVs (Ahn et al., 2021) and platelet-derived EVs (Kaminski et al., 2020), to establish whether neuroprotective and neuroregenerative effects were attributable to a cell of origin specific role of MSC-EVs, rather than EVs in general. Finally, the use of modified EVs either enriched for or depleted of cargo suspected to mediate observed beneficial effects (Chu et al., 2020; Xin et al., 2020; Du et al., 2021) has enabled determination of potential molecular mechanisms that could be exploited in future drug and nanotechnology developments aimed at treating perinatal brain injury. Notably, the addition of more than one control groups in in-vivo studies utilizing EVs as a potential therapeutic strategy for perinatal brain injury has increased since 2018 following publication of the MISEV guidelines, which advocates use of more control groups (Thery et al., 2018).

In relation to appropriate animal models, there are obvious anatomical and physiological differences between species and humans. The use of rodents and the extrapolation of data derived from these models has to be carefully considered since rodents have a lissencephalic (smooth surfaced) brain, with limited white matter tracts (Cook and Tymianski, 2011; Cai and Wang, 2016), that does not resemble the highly gyrencephalic human brain, and which makes interpretation of findings difficult. The fetal sheep model offers considerable advantages to bridge the gap between pre-clinical findings and clinical implementation. Specifically, the chronically instrumented preterm fetal sheep umbilical cord occlusion model closely replicates the common pathophysiological features of human preterm HI, namely diffuse white matter injury, with hippocampal as well as cortical and subcortical neuronal injury, thus emphasizing its translational character (Wassink et al., 2017; Cho et al., 2019a). Secondly, the translational nature of the fetal sheep model is best highlighted by preclinical studies of the near-term in-utero fetal sheep model (Gunn et al., 2005). These studies have provided key evidence of the safety and efficacy of hypothermia for treatment of HI encephalopathy, which is now the only viable therapy for HI encephalopathy in near-term or term infants. However, disadvantages of using such a large animal model include high cost of animal purchase, breeding, surgery, and housing. Consequently, it may not be possible to employ large numbers of animals into trials to demonstrate reproducibility; a factor, which researchers must consider in the pursuit of obtaining meaningful results when generating in-vivo data.

As the therapeutic application of EVs in the treatment of perinatal brain injury is in its infancy, there still are no studies reported which have tested the benefits of EVs in combination with hypothermia or other therapeutics. It is possible that administration of EVs will work synergistically with established therapeutic interventions and enhance clinical outcomes. Consequently, further work should be done to explore the potential of combination therapies.

Finally, although it is beyond the scope of this review, a unifying goal of recent and future advances in the field of EVs is to improve the therapeutic potential of EVs. More so, it is the need to find an effective way to treat newborns with brain injuries, which is one of the greatest challenges facing perinatal medicine. Achieving a promising outcome is most likely dependent on the composition of the EV cargo. As outlined previously, cargo found within EVs can include nucleic acids [miRNA (Valadi et al., 2007; Hunter et al., 2008), lncRNA (Gezer et al., 2014; Abramowicz and Story, 2020; Born et al., 2020), 
mRNA (Valadi et al., 2007; Kalra et al., 2012)], proteins (Simpson et al., 2008; Kalra et al., 2012), and DNA (Cai et al., 2013), and even cell surface receptors, cytosolic and nuclear proteins, and enzymes from the EV cell of origin (Andaloussi et al., 2013; Antonyak and Cerione, 2014; Yoon et al., 2014). Little is known regarding how the composition of cargo is specifically regulated by a particular cell. In all likelihood, there exists specific intracellular mechanisms that regulate the composition (Margolis and Sadovsky, 2019). However, artificial technologies could be implemented to achieve this in several ways; either by way of loading therapeutic cargo (including nucleic acids and small molecules compounds) and/or engineering EVs to display targeting moieties or protein therapeutics simultaneously on their membrane surface (Kooijmans et al., 2016; Antes et al., 2018; Murphy et al., 2019; Gupta et al., 2020). While there are no studies performed in perinatal animal models of brain injury, this approach clearly has the potential to offer considerable advantages around individualization of therapies that will improve neonatal care in the future.

\section{CONCLUSION}

In recent years, there have been substantial efforts made to understand the characteristics of EVs and in particular, the therapeutic potential of those derived from stem cells. This has heralded an awareness that EVs and their cargo can become potential therapeutics in the treatment of brain disorders. However, as evidenced by this review, only a limited number of studies have sought to investigate their capacity to ameliorate the sequelae of perinatal brain injury. Therefore, definitive conclusions cannot be made regarding the use of EVs as a treatment for perinatal brain injury. Nevertheless, the available data demonstrates that therapeutic administration of EVs represents a promising new tool for the treatment of perinatal brain injury with studies utilizing in-vitro and in-vivo models suggesting that EVs will not only prevent, but also repair

\section{REFERENCES}

Abramowicz, A., and Story, M. D. (2020). The long and short of it: the emerging roles of non-coding RNA in small extracellular vesicles. Cancers (Basel) 12:1445. doi: $10.3390 /$ cancers 12061445

Adamiak, M., Cheng, G., Bobis-Wozowicz, S., Zhao, L., Kedracka-Krok, S., Samanta, A., et al. (2018). Induced pluripotent stem cell (iPSC)derived extracellular vesicles are safer and more effective for cardiac repair than iPSCs. Circ. Res. 122, 296-309. doi: 10.1161/circresaha.117.31 1769

Ahn, S. Y., Chang, Y. S., Sung, D. K., Sung, S. I., Ahn, J. Y., and Park, W. S. (2017). Pivotal role of brain-derived neurotrophic factor secreted by mesenchymal stem cells in severe intraventricular hemorrhage in newborn rats. Cell Transplant. 26, 145-156. doi: 10.3727/096368916×692861

Ahn, S. Y., Sung, D. K., Kim, Y. E., Sung, S., Chang, Y. S., and Park, W. S. (2021). Brain-derived neurotropic factor mediates neuroprotection of mesenchymal stem cell-derived extracellular vesicles against severe intraventricular hemorrhage in newborn rats. Stem Cells Trans. Med. 10, 374384. doi: 10.1002/sctm.20-0301

Ajmo, C. T. Jr., Vernon, D. O., Collier, L., Hall, A. A., Garbuzova-Davis, S., Willing, A., et al. (2008). The spleen contributes to stroke-induced neurodegeneration. J. Neurosci. Res. 86, 2227-2234. doi: 10.1002/jnr.21661 brain damage. Collectively, these studies highlight an enormous potential for future EV therapies, which requires further investigation. However, to ensure the validity of results and to advance the field of perinatal EV research, investigators should standardize future research practice by ensuring compliance to current minimal requirements for EV studies (MISEV guidelines) (Thery et al., 2018) and those of preclinical biological medicinal products (Lener et al., 2015). Equally important is the need for more systematic in-vivo studies, regarding the safety and potency of EVs, and the development of large-scale production, which is imperative to enable clinical translation.

\section{AUTHOR CONTRIBUTIONS}

TG conducted the unbiased literature search. TG and MF contributed equally to the preparation and writing of the review article. The final submitted article has been revised and approved by both authors.

\section{FUNDING}

This research was supported by grants awarded to MF from the Neurological Foundation of New Zealand (No. 1830SPG), Auckland Academic Health Alliance (AAHA) Research Collaboration Fund (No. 8752), Health Research Council of New Zealand (No. 18/183), Cure Kids (No. 3581), and Faculty Research Health and Development Fund, The University of Auckland.

\section{SUPPLEMENTARY MATERIAL}

The Supplementary Material for this article can be found online at: https://www.frontiersin.org/articles/10.3389/fnins. 2021.744840/full\#supplementary-material

Albertsson, A. M., Bi, D., Duan, L., Zhang, X., Leavenworth, J. W., Qiao, L., et al. (2014). The immune response after hypoxia-ischemia in a mouse model of preterm brain injury. J. Neuroinflammation 11:153.

Alexandrou, G., Mårtensson, G., Skiöld, B., Blennow, M., Adén, U., and Vollmer, B. (2014). White matter microstructure is influenced by extremely preterm birth and neonatal respiratory factors. Acta Paediatr. 103, 48-56.

Ananth, C. V., Joseph, K. S., Oyelese, Y., Demissie, K., and Vintzileos, A. M. (2005). Trends in preterm birth and perinatal mortality among singletons: United States, 1989 through 2000. Obstet. Gynecol. 105, 1084-1091. doi: 10. 1097/01.aog.0000158124.96300.c7

Andaloussi, S. E. L., Mäger, I., Breakefield, X. O., and Wood, M. J. (2013). Extracellular vesicles: biology and emerging therapeutic opportunities. Nat. Rev. Drug Discov. 12, 347-357. doi: 10.1038/nrd3978

Antes, T. J., Middleton, R. C., Luther, K. M., Ijichi, T., Peck, K. A., Liu, W. J., et al. (2018). Targeting extracellular vesicles to injured tissue using membrane cloaking and surface display. J. Nanobiotechnol. 16:61.

Antonyak, M. A., and Cerione, R. A. (2014). Microvesicles as mediators of intercellular communication in cancer. Methods Mol. Biol. 1165, 147-173. doi: 10.1007/978-1-4939-0856-1_11

Arantes, R. M., and Andrews, N. W. (2006). A role for synaptotagmin VII-regulated exocytosis of lysosomes in neurite outgrowth from primary sympathetic neurons. J. Neurosci. 26, 4630-4637. doi: 10.1523/jneurosci.0009-06.2006 
Azzopardi, D. V., Strohm, B., Edwards, A. D., Dyet, L., Halliday, H. L., Juszczak, E., et al. (2009). Moderate hypothermia to treat perinatal asphyxial encephalopathy. N. Engl. J. Med. 361, 1349-1358.

Azzopardi, D., Strohm, B., Marlow, N., Brocklehurst, P., Deierl, A., Eddama, O., et al. (2014). Effects of hypothermia for perinatal asphyxia on childhood outcomes. N. Engl. J. Med. 371, 140-149.

Baburamani, A. A., Supramaniam, V. G., Hagberg, H., and Mallard, C. (2014). Microglia toxicity in preterm brain injury. Reprod. Toxicol. 48, 106-112. doi: 10.1016/j.reprotox.2014.04.002

Back, S. A. (2014). Cerebral white and gray matter injury in newborns: new insights into pathophysiology and management. Clin. Perinatol. 41, 1-24. doi: 10.1016/ j.clp.2013.11.001

Back, S. A. (2015). Brain injury in the preterm infant: new horizons for pathogenesis and prevention. Pediatr. Neurol. 53, 185-192. doi: 10.1016/j. pediatrneurol.2015.04.006

Back, S. A., and Miller, S. P. (2014). Brain injury in premature neonates: a primary cerebral dysmaturation disorder? Ann. Neurol. 75, 469-486.

Back, S. A., Luo, N. L., Borenstein, N. S., Levine, J. M., Volpe, J. J., and Kinney, H. C. (2001). Late oligodendrocyte progenitors coincide with the developmental window of vulnerability for human perinatal white matter injury. J. Neurosci. 21, 1302-1312. doi: 10.1523/jneurosci.21-04-01302.2001

Bahrini, I., Song, J.-H., Diez, D., and Hanayama, R. (2015). Neuronal exosomes facilitate synaptic pruning by up-regulating complement factors in microglia. Sci. Rep. 5:7989.

Bakhti, M., Winter, C., and Simons, M. (2011). Inhibition of myelin membrane sheath formation by oligodendrocyte-derived exosome-like vesicles. J. Biol. Chem. 286, 787-796. doi: 10.1074/jbc.m110.190009

Ballabh, P., Hu, F., Kumarasiri, M., Braun, A., and Nedergaard, M. (2005). Development of tight junction molecules in blood vessels of germinal matrix, cerebral cortex, and white matter. Pediatr. Res. 58, 791-798. doi: 10.1203/01. pdr.0000180535.14093.fb

Banigan, M. G., Kao, P. F., Kozubek, J. A., Winslow, A. R., Medina, J., Costa, J., et al. (2013). Differential expression of exosomal microRNAs in prefrontal cortices of schizophrenia and bipolar disorder patients. PLoS One 8:e48814. doi: 10.1371/journal.pone.0048814

Baraniak, P. R., and McDevitt, T. C. (2010). Stem cell paracrine actions and tissue regeneration. Regen. Med. 5, 121-143. doi: 10.2217/rme.09.74

Bassler, D., Stoll, B. J., Schmidt, B., Asztalos, E. V., Roberts, R. S., Robertson, C. M., et al. (2009). Using a count of neonatal morbidities to predict poor outcome in extremely low birth weight infants: added role of neonatal infection. Pediatrics 123, 313-318. doi: 10.1542/peds.2008-0377

Batsali, A. K., Kastrinaki, M. C., Papadaki, H. A., and Pontikoglou, C. (2013). Mesenchymal stem cells derived from Wharton's Jelly of the umbilical cord: biological properties and emerging clinical applications. Curr. Stem Cell Res. Ther. 8, 144-155. doi: 10.2174/1574888x11308020005

Beck, S., Wojdyla, D., Say, L., Betran, A. P., Merialdi, M., Requejo, J. H., et al. (2010). The worldwide incidence of preterm birth: a systematic review of maternal mortality and morbidity. Bull. World Health Organ. 88, 31-38. doi: 10.2471/ blt.08.062554

Behrman, R. E., and Butler, A. S. (2007). Preterm Birth: Causes, Consequences, and Prevention. Washington, DC: National Academies Press (US).

Bennet, L., Roelfsema, V., George, S., Dean, J. M., Emerald, B. S., and Gunn, A. J. (2007). The effect of cerebral hypothermia on white and grey matter injury induced by severe hypoxia in preterm fetal sheep. J. Physiol. 578, 491-506. doi: 10.1113/jphysiol.2006.119602

Bianco, F., Perrotta, C., Novellino, L., Francolini, M., Riganti, L., Menna, E., et al. (2009). Acid sphingomyelinase activity triggers microparticle release from glial cells. EMBO J. 28, 1043-1054. doi: 10.1038/emboj.2009.45

Bianco, F., Pravettoni, E., Colombo, A., Schenk, U., Möller, T., Matteoli, M., et al. (2005). Astrocyte-derived ATP induces vesicle shedding and IL-1 beta release from microglia. J. Immunol. 174, 7268-7277. doi: 10.4049/jimmunol.174.11. 7268

Bifrare, Y.-D., Kummer, J., Joss, P., Täuber, M. G., and Leib, S. L. (2005). BrainDerived neurotrophic factor protects against multiple forms of brain injury in bacterial meningitis. J. Infect. Dis. 191, 40-45. doi: 10.1086/426399

Blencowe, H., Cousens, S., Chou, D., Oestergaard, M., Say, L., Moller, A. B., et al. (2013a). Born too soon: the global epidemiology of 15 million preterm births. Reprod. Health 10:S2.
Blencowe, H., Cousens, S., Oestergaard, M. Z., Chou, D., Moller, A. B., Narwal, R., et al. (2012). National, regional, and worldwide estimates of preterm birth rates in the year 2010 with time trends since 1990 for selected countries: a systematic analysis and implications. Lancet 379, 2162-2172. doi: 10.1016/s0140-6736(12) 60820-4

Blencowe, H., Lee, A. C., Cousens, S., Bahalim, A., Narwal, R., Zhong, N., et al. (2013b). Preterm birth-associated neurodevelopmental impairment estimates at regional and global levels for 2010. Pediatr. Res. 74(Suppl. 1), 17-34. doi: 10.1038/pr.2013.204

Bonab, M. M., Alimoghaddam, K., Talebian, F., Ghaffari, S. H., Ghavamzadeh, A., and Nikbin, B. (2006). Aging of mesenchymal stem cell in vitro. BMC Cell Biol. 7:14. doi: $10.1186 / 1471-2121-7-14$

Bonafede, R., Scambi, I., Peroni, D., Potrich, V., Boschi, F., Benati, D., et al. (2016). Exosome derived from murine adipose-derived stromal cells: neuroprotective effect on in vitro model of amyotrophic lateral sclerosis. Exp. Cell Res. 340, 150-158. doi: 10.1016/j.yexcr.2015.12.009

Born, L. J., Harmon, J. W., and Jay, S. M. (2020). Therapeutic potential of extracellular vesicle-associated long noncoding RNA. Bioeng. Transl. Med. 5:e10172.

Briatore, E., Ferrari, F., Pomero, G., Boghi, A., Gozzoli, L., Micciolo, R., et al. (2013). EEG findings in cooled asphyxiated newborns and correlation with site and severity of brain damage. Brain Dev. 35, 420-426. doi: 10.1016/j.braindev.2012. 07.002

Budnik, V., Ruiz-Canada, C., and Wendler, F. (2016). Extracellular vesicles round off communication in the nervous system. Nat. Rev. Neurosci. 17, 160-172. doi: 10.1038/nrn.2015.29

Burbidge, K., Zwikelmaier, V., Cook, B., Long, M. M., Balva, B., Lonigro, M., et al. (2020). Cargo and cell-specific differences in extracellular vesicle populations identified by multiplexed immunofluorescent analysis. J. Extracell. Vesicles 9:1789326. doi: 10.1080/20013078.2020.1789326

Burda, J. E., and Sofroniew, M. V. (2014). Reactive gliosis and the multicellular response to CNS damage and disease. Neuron 81, 229-248.

Buser, J. R., Maire, J., Riddle, A., Gong, X., Nguyen, T., Nelson, K., et al. (2012). Arrested preoligodendrocyte maturation contributes to myelination failure in premature infants. Ann. Neurol. 71, 93-109. doi: 10.1002/ana.22627

Cai, B., and Wang, N. (2016). Large animal stroke models vs. rodent stroke models, pros and cons, and combination? Acta Neurochir. Suppl. 121, 77-81. doi: 10.1007/978-3-319-18497-5_13

Cai, J., Han, Y., Ren, H., Chen, C., He, D., Zhou, L., et al. (2013). Extracellular vesicle-mediated transfer of donor genomic DNA to recipient cells is a novel mechanism for genetic influence between cells. J. Mol. Cell Biol. 5, 227-238. doi: $10.1093 / \mathrm{jmcb} / \mathrm{mjt} 011$

Cai, J., Wu, J., Wang, J., Li, Y., Hu, X., Luo, S., et al. (2020). Extracellular vesicles derived from different sources of mesenchymal stem cells: therapeutic effects and translational potential. Cell Biosci. 10:69.

Caldú, X., Narberhaus, A., Junqué, C., Giménez, M., Vendrell, P., Bargalló, N., et al. (2006). Corpus callosum size and neuropsychologic impairment in adolescents who were born preterm. J. Child Neurol. 21, 406-410. doi: 10.1177/ 08830738060210050801

Campanella, C., Caruso Bavisotto, C., Logozzi, M., Marino Gammazza, A., Mizzoni, D., Cappello, F., et al. (2019). On the choice of the extracellular vesicles for therapeutic purposes. Int. J. Mol. Sci. 20:236. doi: 10.3390/ijms20020236

Can, A., and Karahuseyinoglu, S. (2007). Concise review: human umbilical cord stroma with regard to the source of fetus-derived stem cells. Stem Cells 25, 2886-2895. doi: 10.1634/stemcells.2007-0417

Chen, A., Xiong, L. J., Tong, Y., and Mao, M. (2013). The neuroprotective roles of BDNF in hypoxic ischemic brain injury. Biomed. Rep. 1, 167-176. doi: 10.3892/br.2012.48

Chen, C. C., Liu, L., Ma, F., Wong, C. W., Guo, X. E., Chacko, J. V., et al. (2016). Elucidation of exosome migration across the blood-brain barrier model in vitro. Cell. Mol. Bioeng. 9, 509-529. doi: 10.1007/s12195-016-0458-3

Chen, S., Datta-Chaudhuri, A., Deme, P., Dickens, A., Dastgheyb, R., Bhargava, P., et al. (2019). Lipidomic characterization of extracellular vesicles in human serum. J. Circ. Biomark. 8:1849454419879848.

Chen, X., Threlkeld, S. W., Cummings, E. E., Juan, I., Makeyev, O., Besio, W. G., et al. (2012). Ischemia-reperfusion impairs blood-brain barrier function and alters tight junction protein expression in the ovine fetus. Neuroscience 226, 89-100. doi: 10.1016/j.neuroscience.2012.08.043 
Cho, K. H. T., Wassink, G., Galinsky, R., Xu, B., Mathai, S., Dhillon, S. K., et al. (2019a). Protective effects of delayed intraventricular TLR7 agonist administration on cerebral white and gray matter following asphyxia in the preterm fetal sheep. Sci. Rep. 9:9562.

Cho, K. H. T., Xu, B., Blenkiron, C., and Fraser, M. (2019b). Emerging roles of miRNAs in brain development and perinatal brain injury. Front. Physiol. 10:227. doi: $10.3389 /$ fphys.2019.00227

Chu, X., Liu, D., Li, T., Ke, H., Xin, D., Wang, S., et al. (2020). Hydrogen sulfidemodified extracellular vesicles from mesenchymal stem cells for treatment of hypoxic-ischemic brain injury. J. Control. Release 328, 13-27. doi: 10.1016/j. jconrel.2020.08.037

Cocucci, E., and Meldolesi, J. (2015). Ectosomes and exosomes: shedding the confusion between extracellular vesicles. Trends Cell Biol. 25, 364-372. doi: 10.1016/j.tcb.2015.01.004

Coleman, B. M., and Hill, A. F. (2015). Extracellular vesicles - Their role in the packaging and spread of misfolded proteins associated with neurodegenerative diseases. Semin. Cell Dev. Biol. 40, 89-96. doi: 10.1016/j.semcdb.2015.02.007

Cook, D. J., and Tymianski, M. (2011). Translating promising preclinical neuroprotective therapies to human stroke trials. Expert Rev. Cardiovasc. Ther. 9, 433-449. doi: 10.1586/erc.11.34

Coolen, M., Katz, S., and Bally-Cuif, L. (2013). miR-9: a versatile regulator of neurogenesis. Front. Cell. Neurosci. 7:220. doi: 10.3389/fncel.2013.00220

Crowther, C. A., Middleton, P. F., Voysey, M., Askie, L., Duley, L., Pryde, P. G., et al. (2017). Assessing the neuroprotective benefits for babies of antenatal magnesium sulphate: an individual participant data meta-analysis. PLoS Med. 14:e1002398. doi: 10.1371/journal.pmed.1002398

Dabrowska, S., Sypecka, J., Jablonska, A., Strojek, L., Wielgos, M., DomanskaJanik, K., et al. (2018). Neuroprotective potential and paracrine activity of stromal Vs. culture-expanded hMSC derived from wharton jelly under cocultured with hippocampal organotypic slices. Mol. Neurobiol. 55, 6021-6036. doi: 10.1007/s12035-017-0802-1

Danzer, K. M., Kranich, L. R., Ruf, W. P., Cagsal-Getkin, O., Winslow, A. R., Zhu, L., et al. (2012). Exosomal cell-to-cell transmission of alpha synuclein oligomers. Mol. Neurodegener. 7:42. doi: 10.1186/1750-1326-7-42

de Almeida, P. E., Ransohoff, J. D., Nahid, A., and Wu, J. C. (2013). Immunogenicity of pluripotent stem cells and their derivatives. Circ. Res. 112, 549-561. doi: 10.1161/circresaha.111.249243

de Haan, T. R., Bijleveld, Y. A., Van Der Lee, J. H., Groenendaal, F., Van Den Broek, M. P., Rademaker, C. M., et al. (2012). Pharmacokinetics and pharmacodynamics of medication in asphyxiated newborns during controlled hypothermia. The PharmaCool multicenter study. BMC Pediatr. 12:45. doi: 10.1186/1471-2431-12-45

de Paula, S., Greggio, S., and Dacosta, J. C. (2010). Use of stem cells in perinatal asphyxia: from bench to bedside. J. Pediatr. (Rio J) 86, 451-464. doi: 10.2223/ jped.2035

de Rivero Vaccari, J. P., Brand, F. III, Adamczak, S., Lee, S. W., Perez-Barcena, J., Wang, M. Y., et al. (2016). Exosome-mediated inflammasome signaling after central nervous system injury. J. Neurochem. 136(Suppl. 1), 39-48. doi: 10.1111/jnc. 13036

Deng, M., Xiao, H., Peng, H., Yuan, H., Xu, Y., Zhang, G., et al. (2018). Preservation of neuronal functions by exosomes derived from different human neural cell types under ischemic conditions. Eur. J. Neurosci. 47, 150-157. doi: 10.1111/ ejn. 13784

Deng, Y. Y., Lu, J., Ling, E. A., and Kaur, C. (2010). Microglia-derived macrophage colony stimulating factor promotes generation of proinflammatory cytokines by astrocytes in the periventricular white matter in the hypoxic neonatal brain. Brain Pathol. 20, 909-925.

Dharap, A., Bowen, K., Place, R., Li, L. C., and Vemuganti, R. (2009). Transient focal ischemia induces extensive temporal changes in rat cerebral microRNAome. J. Cereb. Blood Flow Metab. 29, 675-687. doi: 10.1038/jcbfm. 2008.157

Dhillon, S. K., Gunn, A. J., Jung, Y., Mathai, S., Bennet, L., and Fraser, M. (2015). Lipopolysaccharide-Induced preconditioning attenuates apoptosis and differentially regulates TLR4 and TLR7 gene expression after ischemia in the preterm ovine fetal brain. Dev. Neurosci. 37, 497-514. doi: 10.1159/000433422

Dickens, A. M., Tovar, Y. R. L. B., Yoo, S. W., Trout, A. L., Bae, M., Kanmogne, M., et al. (2017). Astrocyte-shed extracellular vesicles regulate the peripheral leukocyte response to inflammatory brain lesions. Sci. Signal. 10:eaai7696. doi: 10.1126/scisignal.aai7696

Doeppner, T. R., Herz, J., Gorgens, A., Schlechter, J., Ludwig, A. K., Radtke, S., et al. (2015). Extracellular vesicles improve post-stroke neuroregeneration and prevent postischemic immunosuppression. Stem Cells Transl. Med. 4, 1131-1143. doi: 10.5966/sctm.2015-0078

Dolcetti, E., Bruno, A., Guadalupi, L., Rizzo, F. R., Musella, A., Gentile, A., et al. (2020). Emerging role of extracellular vesicles in the pathophysiology of multiple sclerosis. Int. J. Mol. Sci. 21:7336. doi: 10.3390/ijms2119 7336

Donders, R., Bogie, J. F. J., Ravanidis, S., Gervois, P., Vanheusden, M., Marée, R., et al. (2018). Human Wharton's jelly-derived stem cells display a distinct immunomodulatory and proregenerative transcriptional signature compared to bone marrow-derived stem cells. Stem Cells Dev. 27, 65-84. doi: 10.1089/ scd.2017.0029

Donega, V., Nijboer, C. H., Van Tilborg, G., Dijkhuizen, R. M., Kavelaars, A., and Heijnen, C. J. (2014). Intranasally administered mesenchymal stem cells promote a regenerative niche for repair of neonatal ischemic brain injury. Exp. Neurol. 261, 53-64. doi: 10.1016/j.expneurol.2014.06.009

Drago, F., Lombardi, M., Prada, I., Gabrielli, M., Joshi, P., Cojoc, D., et al. (2017). ATP modifies the proteome of extracellular vesicles released by microglia and influences their action on astrocytes. Front. Pharmacol. 8:910. doi: 10.3389/ fphar.2017.00910

Drommelschmidt, K., Serdar, M., Bendix, I., Herz, J., Bertling, F., Prager, S., et al. (2017). Mesenchymal stem cell-derived extracellular vesicles ameliorate inflammation-induced preterm brain injury. Brain Behav. Immun. 60, 220-232. doi: 10.1016/j.bbi.2016.11.011

Du, L., Jiang, Y., and Sun, Y. (2021). Astrocyte-derived exosomes carry microRNA$17-5 \mathrm{p}$ to protect neonatal rats from hypoxic-ischemic brain damage via inhibiting BNIP-2 expression. NeuroToxicology 83, 28-39. doi: 10.1016/j.neuro. 2020.12.006

Emmanouilidou, E., Melachroinou, K., Roumeliotis, T., Garbis, S. D., Ntzouni, M., Margaritis, L. H., et al. (2010). Cell-produced alpha-synuclein is secreted in a calcium-dependent manner by exosomes and impacts neuronal survival. J. Neurosci. 30, 6838-6851. doi: 10.1523/jneurosci.5699-09.2010

Engelhardt, E., Inder, T. E., Alexopoulos, D., Dierker, D. L., Hill, J., Van Essen, D., et al. (2015). Regional impairments of cortical folding in premature infants. Ann. Neurol. 77, 154-162. doi: 10.1002/ana.24313

Feliciano, D. M., Zhang, S., Nasrallah, C. M., Lisgo, S. N., and Bordey, A. (2014). Embryonic cerebrospinal fluid nanovesicles carry evolutionarily conserved molecules and promote neural stem cell amplification. PLoS One 9:e88810. doi: 10.1371/journal.pone.0088810

Fevrier, B., Vilette, D., Archer, F., Loew, D., Faigle, W., Vidal, M., et al. (2004). Cells release prions in association with exosomes. Proc. Natl. Acad. Sci. U.S.A. 101, 9683-9688. doi: 10.1073/pnas.0308413101

Fiandaca, M. S., Kapogiannis, D., Mapstone, M., Boxer, A., Eitan, E., Schwartz, J. B., et al. (2015). Identification of preclinical Alzheimer's disease by a profile of pathogenic proteins in neurally derived blood exosomes: a case-control study. Alzheimers Dement. 11, 600-607.e601.

Fischer, U. M., Harting, M. T., Jimenez, F., Monzon-Posadas, W. O., Xue, H., Savitz, S. I., et al. (2009). Pulmonary passage is a major obstacle for intravenous stem cell delivery: the pulmonary first-pass effect. Stem Cells Dev. 18, 683-692. doi: $10.1089 /$ scd.2008.0253

Fitzner, D., Schnaars, M., Van Rossum, D., Krishnamoorthy, G., Dibaj, P., Bakhti, M., et al. (2011). Selective transfer of exosomes from oligodendrocytes to microglia by macropinocytosis. J. Cell Sci. 124, 447-458. doi: 10.1242/jcs. 074088

Fraser, M., Bennet, L., Gunning, M., Williams, C., Gluckman, P. D., George, S., et al. (2005). Cortical electroencephalogram suppression is associated with postischemic cortical injury in 0.65 gestation fetal sheep. Brain Res. Dev. Brain Res. 154, 45-55. doi: 10.1016/j.devbrainres.2004.10.002

Fraser, M., Bennet, L., Helliwell, R., Wells, S., Williams, C., Gluckman, P., et al. (2007). Regional specificity of magnetic resonance imaging and histopathology following cerebral ischemia in preterm fetal sheep. Reprod. Sci. 14, 182-191. doi: 10.1177/1933719107299612

Fraser, M., Bennet, L., Van Zijl, P. L., Mocatta, T. J., Williams, C. E., Gluckman, P. D., et al. (2008). Extracellular amino acids and lipid peroxidation products in 
periventricular white matter during and after cerebral ischemia in preterm fetal sheep. J. Neurochem. 105, 2214-2223. doi: 10.1111/j.1471-4159.2008.05313.x

Frausin, S., Viventi, S., Verga Falzacappa, L., Quattromani, M. J., Leanza, G., Tommasini, A., et al. (2015). Wharton's jelly derived mesenchymal stromal cells: biological properties, induction of neuronal phenotype and current applications in neurodegeneration research. Acta Histochem. 117, 329-338.

Fröhlich, D., Kuo, W. P., Frühbeis, C., Sun, J. J., Zehendner, C. M., Luhmann, H. J., et al. (2014). Multifaceted effects of oligodendroglial exosomes on neurons: impact on neuronal firing rate, signal transduction and gene regulation. Philos. Trans. R. Soc. Lond. B Biol. Sci. 369:20130510. doi: 10.1098/rstb.2013.0510

Frühbeis, C., Fröhlich, D., Kuo, W. P., Amphornrat, J., Thilemann, S., Saab, A. S., et al. (2013a). Neurotransmitter-triggered transfer of exosomes mediates oligodendrocyte-neuron communication. PLoS Biol. 11:e1001604. doi: 10.1371/ journal.pbio. 1001604

Frühbeis, C., Fröhlich, D., Kuo, W. P., and Krämer-Albers, E.-M. (2013b). Extracellular vesicles as mediators of neuron-glia communication. Front. Cell. Neurosci. 7:182. doi: 10.3389/fncel.2013.00182

Fu, Y., Karbaat, L., Wu, L., Leijten, J., Both, S. K., and Karperien, M. (2017). Trophic effects of mesenchymal stem cells in tissue regeneration. Tissue Eng. Part B Rev. 23, 515-528.

Galinsky, R., Lear, C. A., Dean, J. M., Wassink, G., Dhillon, S. K., Fraser, M., et al. (2018). Complex interactions between hypoxia-ischemia and inflammation in preterm brain injury. Dev. Med. Child Neurol. 60, 126-133. doi: 10.1111/dmcn. 13629

Gezer, U., Özgür, E., Cetinkaya, M., Isin, M., and Dalay, N. (2014). Long noncoding RNAs with low expression levels in cells are enriched in secreted exosomes. Cell Biol. Int. 38, 1076-1079.

Gluckman, P. D., Wyatt, J. S., Azzopardi, D., Ballard, R., Edwards, A. D., Ferriero, D. M., et al. (2005). Selective head cooling with mild systemic hypothermia after neonatal encephalopathy: multicentre randomised trial. Lancet 365, 663-670. doi: 10.1016/s0140-6736(05)70932-6

Goetzl, L., Darbinian, N., and Goetzl, E. J. (2016). Novel window on early human neurodevelopment via fetal exosomes in maternal blood. Ann. Clin. Transl. Neurol. 3, 381-385. doi: 10.1002/acn3.296

Goetzl, L., Darbinian, N., and Merabova, N. (2019). Noninvasive assessment of fetal central nervous system insult: potential application to prenatal diagnosis. Prenat. Diagn. 39, 609-615. doi: 10.1002/pd.5474

Goetzl, L., Merabova, N., Darbinian, N., Martirosyan, D., Poletto, E., Fugarolas, K., et al. (2017). Diagnostic potential of neural exosome cargo as biomarkers for acute brain injury. Ann. Clin. Transl. Neurol. 5, 4-10. doi: 10.1002/acn3.499

Gomzikova, M. O., James, V., and Rizvanov, A. A. (2019). Therapeutic application of mesenchymal stem cells derived extracellular vesicles for immunomodulation. Front. Immunol. 10:2663. doi: 10.3389/fimmu.2019. 02663

Gong, J., Körner, R., Gaitanos, L., and Klein, R. (2016). Exosomes mediate cell contact-independent ephrin-Eph signaling during axon guidance. J. Cell Biol. 214, 35-44. doi: 10.1083/jcb.201601085

Gopagondanahalli, K. R., Li, J., Fahey, M. C., Hunt, R. W., Jenkin, G., Miller, S. L., et al. (2016). Preterm hypoxic-ischemic encephalopathy. Front. Pediatr. 4:114. doi: $10.3389 /$ fped.2016.00114

Gould, S. J., and Raposo, G. (2013). As we wait: coping with an imperfect nomenclature for extracellular vesicles. J. Extracell. Vesicles 2:20389. doi: 10. 3402/jev.v2i0.20389

Gross, E. A., Swenberg, J. A., Fields, S., and Popp, J. A. (1982). Comparative morphometry of the nasal cavity in rats and mice. J. Anat. 135, 83-88.

Gunn, A. J., Battin, M., Gluckman, P. D., Gunn, T. R., and Bennet, L. (2005). Therapeutic hypothermia: from lab to NICU. J. Perinat. Med. 33, 340-346.

Guo, Y., Tan, J., Miao, Y., Sun, Z., and Zhang, Q. (2019). Effects of microvesicles on cell apoptosis under hypoxia. Oxid. Med. Cell. Longev. 2019:5972152.

Gupta, D., Wiklander, O. P. B., Görgens, A., Conceição, M., Corso, G., Liang, X., et al. (2020). Engineering of extracellular vesicles for display of protein biotherapeutics. bioRxiv [preprint]. doi: 10.1101/2020.06.14.149823

Gussenhoven, R., Klein, L., Ophelders, D., Habets, D. H. J., Giebel, B., Kramer, B. W., et al. (2019). Annexin A1 as neuroprotective determinant for bloodbrain barrier integrity in neonatal hypoxic-ischemic encephalopathy. J. Clin. Med. 8:137. doi: 10.3390/jcm8020137

Hagberg, H., Gilland, E., Bona, E., Hanson, L. Å, Hahn-Zoric, M., Blennow, M., et al. (1996). Enhanced expression of interleukin (IL)-1 and IL-6 messenger
RNA and bioactive protein after hypoxia-ischemia in neonatal rats. Pediatr. Res. 40, 603-609. doi: 10.1203/00006450-199610000-00015

Hagberg, H., Mallard, C., Ferriero, D. M., Vannucci, S. J., Levison, S. W., Vexler, Z. S., et al. (2015). The role of inflammation in perinatal brain injury. Nat. Rev. Neurol. 11, 192-208.

Hajj, G. N., Arantes, C. P., Dias, M. V., Roffé, M., Costa-Silva, B., Lopes, M. H., et al. (2013). The unconventional secretion of stress-inducible protein 1 by a heterogeneous population of extracellular vesicles. Cell. Mol. Life Sci. 70, 3211-3227. doi: 10.1007/s00018-013-1328-y

Hammad, A., Westacott, L., and Zaben, M. (2018). The role of the complement system in traumatic brain injury: a review. J. Neuroinflammation 15:24.

Han, J., Yang, S., Hao, X., Zhang, B., Zhang, H., Xin, C., et al. (2020). Extracellular vesicle-derived microRNA-410 from mesenchymal stem cells protects against neonatal hypoxia-ischemia brain damage through an HDAC1-dependent EGR2/Bcl2 Axis. Front. Cell Dev. Biol. 8:579236. doi: 10.3389/fcell.2020.579236

Hanson, L. R., and Frey, W. H. II (2008). Intranasal delivery bypasses the bloodbrain barrier to target therapeutic agents to the central nervous system and treat neurodegenerative disease. BMC Neurosci. 9(Suppl. 3):S5. doi: 10.1186/14712202-9-S3-S5

Huang, Y., Liu, Z., Tan, F., Hu, Z., and Lu, M. (2020). Effects of the insulted neuronal cells-derived extracellular vesicles on the survival of umbilical cordderived mesenchymal stem cells following cerebral ischemia/reperfusion injury. Oxid. Med. Cell. Longev. 2020:9768713.

Hunter, M. P., Ismail, N., Zhang, X., Aguda, B. D., Lee, E. J., Yu, L., et al. (2008). Detection of microRNA expression in human peripheral blood microvesicles. PLoS One 3:e3694. doi: 10.1371/journal.pone.0003694

Iadecola, C., and Anrather, J. (2011). The immunology of stroke: from mechanisms to translation. Nat. Med. 17, 796-808. doi: 10.1038/nm.2399

Ikeda, T. (2008). Stem cells and neonatal brain injury. Cell Tissue Res. 331, 263-269. doi: 10.1007/s00441-007-0546-8

Ilancheran, S., Michalska, A., Peh, G., Wallace, E. M., Pera, M., and Manuelpillai, U. (2007). Stem cells derived from human fetal membranes display multi-lineage differentiation potential. Biol. Reprod. 77, 577-588. doi: 10.1095/biolreprod. 106.055244

Inder, T. E., Anderson, N. J., Spencer, C., Wells, S., and Volpe, J. J. (2003a). White matter injury in the premature infant: a comparison between serial cranial sonographic and MR findings at term. AJNR Am. J. Neuroradiol. 24, 805-809.

Inder, T. E., Buckland, L., Williams, C. E., Spencer, C., Gunning, M. I., Darlow, B. A., et al. (2003b). Lowered electroencephalographic spectral edge frequency predicts the presence of cerebral white matter injury in premature infants. Pediatrics 111, 27-33. doi: 10.1542/peds.111.1.27

Inder, T. E., Huppi, P. S., Warfield, S., Kikinis, R., Zientara, G. P., Barnes, P. D., et al. (1999). Periventricular white matter injury in the premature infant is followed by reduced cerebral cortical gray matter volume at term. Ann. Neurol. 46, 755-760. doi: 10.1002/1531-8249(199911)46:5<755::aid-ana11>3.0.co;2-0

Inder, T. E., Warfield, S. K., Wang, H., Hüppi, P. S., and Volpe, J. J. (2005). Abnormal cerebral structure is present at term in premature infants. Pediatrics 115, 286-294. doi: 10.1542/peds.2004-0326

Irmady, K., Jackman, K. A., Padow, V. A., Shahani, N., Martin, L. A., Cerchietti, L., et al. (2014). Mir-592 regulates the induction and cell death-promoting activity of p75NTR in neuronal ischemic injury. J. Neurosci. 34, 3419-3428. doi: 10.1523/jneurosci.1982-13.2014

Izadpanah, R., Kaushal, D., Kriedt, C., Tsien, F., Patel, B., Dufour, J., et al. (2008). Long-term in vitro expansion alters the biology of adult mesenchymal stem cells. Cancer Res. 68, 4229-4238. doi: 10.1158/0008-5472.can-07-5272

Jacobs, S. E., Berg, M., Hunt, R., Tarnow-Mordi, W. O., Inder, T. E., and Davis, P. G. (2013). Cooling for newborns with hypoxic ischaemic encephalopathy. Cochrane Database Syst. Rev. 2013:CD003311.

Jacobs, S. E., Morley, C. J., Inder, T. E., Stewart, M. J., Smith, K. R., Mcnamara, P. J., et al. (2011). Whole-body hypothermia for term and near-term newborns with hypoxic-ischemic encephalopathy: a randomized controlled trial. Arch. Pediatr. Adolesc. Med. 165, 692-700. doi: 10.1001/archpediatrics.2011.43

Jarmalavičiūtè, A., Tunaitis, V., Pivoraitè, U., Venalis, A., and Pivoriūnas, A. (2015). Exosomes from dental pulp stem cells rescue human dopaminergic neurons from 6-hydroxy-dopamine-induced apoptosis. Cytotherapy 17, 932939. doi: 10.1016/j.jcyt.2014.07.013

Jellema, R. K., Lima Passos, V., Zwanenburg, A., Ophelders, D. R., De Munter, S., Vanderlocht, J., et al. (2013a). Cerebral inflammation and mobilization of the 
peripheral immune system following global hypoxia-ischemia in preterm sheep. J. Neuroinflammation 10:13. doi: 10.1111/jpc.13882_26

Jellema, R. K., Wolfs, T. G., Lima Passos, V., Zwanenburg, A., Ophelders, D. R., Kuypers, E., et al. (2013b). Mesenchymal stem cells induce T-cell tolerance and protect the preterm brain after global hypoxia-ischemia. PLoS One 8:e73031. doi: 10.1371/journal.pone.0073031

Jeppesen, D. K., Fenix, A. M., Franklin, J. L., Higginbotham, J. N., Zhang, Q., Zimmerman, L. J., et al. (2019). Reassessment of exosome composition. Cell 177, 428-445.e418.

Jiang, Y., Zhu, J., Xu, G., and Liu, X. (2011). Intranasal delivery of stem cells to the brain. Expert Opin. Drug Deliv. 8, 623-632. doi: 10.1517/17425247.2011.566267

Joerger-Messerli, M. S., Oppliger, B., Spinelli, M., Thomi, G., Di Salvo, I., Schneider, P., et al. (2018). Extracellular vesicles derived from Wharton's jelly mesenchymal stem cells prevent and resolve programmed cell death mediated by perinatal hypoxia-ischemia in neuronal cells. Cell Transplant. 27, 168-180. doi: $10.1177 / 0963689717738256$

Joshi, P., Turola, E., Ruiz, A., Bergami, A., Libera, D. D., Benussi, L., et al. (2014). Microglia convert aggregated amyloid- $\beta$ into neurotoxic forms through the shedding of microvesicles. Cell Death Differ. 21, 582-593. doi: 10.1038/cdd. 2013.180

Juul, S. E., and Pet, G. C. (2015). Erythropoietin and neonatal neuroprotection. Clin. Perinatol. 42, 469-481. doi: 10.1016/j.clp.2015.04.004

Kadhim, H., Tabarki, B., Verellen, G., De Prez, C., Rona, A. M., and Sébire, G. (2001). Inflammatory cytokines in the pathogenesis of periventricular leukomalacia. Neurology 56, 1278-1284. doi: 10.1212/wnl.56.10.1278

Kalra, H., Simpson, R. J., Ji, H., Aikawa, E., Altevogt, P., Askenase, P., et al. (2012). Vesiclepedia: a compendium for extracellular vesicles with continuous community annotation. PLoS Biol. 10:e1001450. doi: 10.1371/journal.pbio. 1001450

Kaminski, N., Köster, C., Mouloud, Y., Börger, V., Felderhoff-Müser, U., Bendix, I., et al. (2020). Mesenchymal stromal cell-derived extracellular vesicles reduce neuroinflammation, promote neural cell proliferation and improve oligodendrocyte maturation in neonatal hypoxic-ischemic brain injury. Front. Cell. Neurosci. 14:601176. doi: 10.3389/fncel.2020.601176

Kennea, N. L., and Mehmet, H. (2004). Perinatal applications of neural stem cells. Best Pract. Res. Clin. Obstet. Gynaecol. 18, 977-994.

Keunen, K., Kersbergen, K. J., Groenendaal, F., Isgum, I., De Vries, L. S., and Benders, M. J. (2012). Brain tissue volumes in preterm infants: prematurity, perinatal risk factors and neurodevelopmental outcome: a systematic review. J. Matern. Fetal Neonatal Med. 25, 89-100. doi: 10.3109/14767058.2012.66 4343

Khwaja, O., and Volpe, J. J. (2008). Pathogenesis of cerebral white matter injury of prematurity. Arch. Dis. Child. Fetal Neonatal Ed. 93, F153-F161.

Kim, E. S., Ahn, S. Y., Im, G. H., Sung, D. K., Park, Y. R., Choi, S. H., et al. (2012). Human umbilical cord blood-derived mesenchymal stem cell transplantation attenuates severe brain injury by permanent middle cerebral artery occlusion in newborn rats. Pediatr. Res. 72, 277-284. doi: 10.1038/pr.2012.71

Kinney, H. C., Haynes, R. L., Xu, G., Andiman, S. E., Folkerth, R. D., Sleeper, L. A., et al. (2012). Neuron deficit in the white matter and subplate in periventricular leukomalacia. Ann. Neurol. 71, 397-406. doi: 10.1002/ana.22612

Ko, H. R., Ahn, S. Y., Chang, Y. S., Hwang, I., Yun, T., Sung, D. K., et al. (2018). Human UCB-MSCs treatment upon intraventricular hemorrhage contributes to attenuate hippocampal neuron loss and circuit damage through BDNFCREB signaling. Stem Cell Res. Ther. 9:326.

Kodali, M., Castro, O. W., Kim, D.-K., Thomas, A., Shuai, B., Attaluri, S., et al. (2019). Intranasally administered human MSC-derived extracellular vesicles pervasively incorporate into neurons and microglia in both intact and status epilepticus injured forebrain. Int. J. Mol. Sci. 21:181. doi: 10.3390/ijms21010181

Kooijmans, S. A. A., Fliervoet, L. A. L., Van Der Meel, R., Fens, M., Heijnen, H. F. G., Van Bergen En Henegouwen, P. M. P., et al. (2016). PEGylated and targeted extracellular vesicles display enhanced cell specificity and circulation time. J. Control. Release 224, 77-85. doi: 10.1016/j.jconrel.2016.01.009

Korkut, C., Li, Y., Koles, K., Brewer, C., Ashley, J., Yoshihara, M., et al. (2013). Regulation of postsynaptic retrograde signaling by presynaptic exosome release. Neuron 77, 1039-1046. doi: 10.1016/j.neuron.2013.01.013

Krämer-Albers, E. M., Bretz, N., Tenzer, S., Winterstein, C., Möbius, W., Berger, H., et al. (2007). Oligodendrocytes secrete exosomes containing major myelin and stress-protective proteins: trophic support for axons? Proteomics Clin. Appl. 1, 1446-1461. doi: 10.1002/prca.200700522

Kumar, A., Mittal, R., Khanna, H. D., and Basu, S. (2008). Free radical injury and blood-brain barrier permeability in hypoxic-ischemic encephalopathy. Pediatrics 122, e722-e727.

Lappalainen, R. S., Narkilahti, S., Huhtala, T., Liimatainen, T., Suuronen, T., Narvanen, A., et al. (2008). The SPECT imaging shows the accumulation of neural progenitor cells into internal organs after systemic administration in middle cerebral artery occlusion rats. Neurosci Lett 440, 246-250. doi: 10.1016/ j.neulet.2008.05.090

Lau, L. T., and Yu, A. C. (2001). Astrocytes produce and release interleukin-1, interleukin-6, tumor necrosis factor alpha and interferon-gamma following traumatic and metabolic injury. J. Neurotrauma 18, 351-359. doi: 10.1089/ 08977150151071035

Lee, J. Y., and Kim, H.-S. (2017). Extracellular vesicles in neurodegenerative diseases: a double-edged sword. Tissue Eng. Regen. Med. 14, 667-678. doi: 10.1007/s13770-017-0090-x

Lee, S. J., Hatran, D. P., Tomimatsu, T., Peña, J. P., Mcauley, G., and Longo, L. D. (2009). Fetal cerebral blood flow, electrocorticographic activity, and oxygenation: responses to acute hypoxia. J. Physiol. 587, 2033-2047. doi: 10. 1113/jphysiol.2009.166983

Lee, W. L. A., Michael-Titus, A. T., and Shah, D. K. (2017). Hypoxic-Ischaemic encephalopathy and the blood-brain barrier in neonates. Dev. Neurosci. 39, 49-58. doi: 10.1159/000467392

Lener, T., Gimona, M., Aigner, L., Börger, V., Buzas, E., Camussi, G., et al. (2015). Applying extracellular vesicles based therapeutics in clinical trials - an ISEV position paper. J. Extracell. Vesicles 4:30087.

Leuchter, R. H., Gui, L., Poncet, A., Hagmann, C., Lodygensky, G. A., Martin, E., et al. (2014). Association between early administration of high-dose erythropoietin in preterm infants and brain MRI abnormality at termequivalent age. JAMA 312, 817-824. doi: 10.1001/jama.2014.9645

Leviton, A., and Gressens, P. (2007). Neuronal damage accompanies perinatal white-matter damage. Trends Neurosci. 30, 473-478. doi: 10.1016/j.tins.2007. 05.009

Lewis, S. (2013). Glia: transporting cargo from A to B. Nat. Rev. Neurosci. 14:589. doi: $10.1038 / \mathrm{nrn} 3568$

Li, H., Niederkorn, J., Neelam, S., Mayhew, E., Word, R., Mcculley, J., et al. (2005). Immunosuppressive factors secreted by human amniotic epithelial cells. Invest. Ophthalmol. Vis. Sci. 46, 900-907. doi: 10.1167/iovs.04-0495

Lieberman, M. A., and Glaser, L. (1981). Density-dependent regulation of cell growth: an example of a cell-cell recognition phenomenon. J. Membr. Biol. 63, 1-11. doi: 10.1007/bf01969440

Liu, W., Wang, Y., Gong, F., Rong, Y., Luo, Y., Tang, P., et al. (2019). Exosomes derived from bone mesenchymal stem cells repair traumatic spinal cord injury by suppressing the activation of Al neurotoxic reactive astrocytes. J. Neurotrauma 36, 469-484. doi: 10.1089/neu.2018.5835

Lombardi, M., Parolisi, R., Scaroni, F., Bonfanti, E., Gualerzi, A., Gabrielli, M., et al. (2019). Detrimental and protective action of microglial extracellular vesicles on myelin lesions: astrocyte involvement in remyelination failure. Acta Neuropathol. 138, 987-1012. doi: 10.1007/s00401-019-02049-1

Luarte, A., Batiz, L. F., Wyneken, U., and Lafourcade, C. (2016). Potential therapies by stem cell-derived exosomes in CNS diseases: focusing on the neurogenic niche. Stem Cells Int. 2016:5736059.

Lutz, I. C., Allegaert, K., De Hoon, J. N., and Marynissen, H. (2020). Pharmacokinetics during therapeutic hypothermia for neonatal hypoxic ischaemic encephalopathy: a literature review. BMJ Paediatr. Open 4:e000685. doi: 10.1136/bmjpo-2020-000685

Ma, S., Xie, N., Li, W., Yuan, B., Shi, Y., and Wang, Y. (2014). Immunobiology of mesenchymal stem cells. Cell Death Differ. 21, 216-225.

Ma, Y., Wang, K., Pan, J., Fan, Z., Tian, C., Deng, X., et al. (2019). Induced neural progenitor cells abundantly secrete extracellular vesicles and promote the proliferation of neural progenitors via extracellular signal-regulated kinase pathways. Neurobiol. Dis. 124, 322-334. doi: 10.1016/j.nbd.2018.12.003

Mallard, C., Davidson, J. O., Tan, S., Green, C. R., Bennet, L., Robertson, N. J., et al. (2014). Astrocytes and microglia in acute cerebral injury underlying cerebral palsy associated with preterm birth. Pediatr. Res. 75, 234-240. doi: 10.1038/pr. 2013.188 
Manaenko, A., Lekic, T., Barnhart, M., Hartman, R., and Zhang, J. H. (2014). Inhibition of transforming growth factor- $\beta$ attenuates brain injury and neurological deficits in a rat model of germinal matrix hemorrhage. Stroke 45 , 828-834. doi: 10.1161/strokeaha.113.003754

March of Dimes, Pmnch, Save the Children, and Who. (2012). in Born Too Soon: The Global Action Report on Preterm Birth, eds C. Howson, M. Kinney, and J. Lawn Geneva: World Health Organisation.

Marcus, M. E., and Leonard, J. N. (2013). FedExosomes: engineering therapeutic biological nanoparticles that truly deliver. Pharmaceuticals (Basel, Switzerland) 6, 659-680. doi: 10.3390/ph6050659

Margolis, L., and Sadovsky, Y. (2019). The biology of extracellular vesicles: the known unknowns. PLoS Biol. 17:e3000363. doi: 10.1371/journal.pbio.300 0363

Martin, C. R., Dammann, O., Allred, E. N., Patel, S., O’shea, T. M., Kuban, K. C., et al. (2010). Neurodevelopment of extremely preterm infants who had necrotizing enterocolitis with or without late bacteremia. J. Pediatr. 157, 751-756.e751.

Mateescu, B., Kowal, E. J., Van Balkom, B. W., Bartel, S., Bhattacharyya, S. N., Buzás, E. I., et al. (2017). Obstacles and opportunities in the functional analysis of extracellular vesicle RNA - an ISEV position paper. J. Extracell. Vesicles 6:1286095. doi: 10.1080/20013078.2017.1286095

Mathieu, M., Martin-Jaular, L., Lavieu, G., and Thery, C. (2019). Specificities of secretion and uptake of exosomes and other extracellular vesicles for cellto-cell communication. Nat. Cell Biol. 21, 9-17. doi: 10.1038/s41556-018-0 250-9

Matsumoto, J., Stewart, T., Sheng, L., Li, N., Bullock, K., Song, N., et al. (2017). Transmission of alpha-synuclein-containing erythrocyte-derived extracellular vesicles across the blood-brain barrier via adsorptive mediated transcytosis: another mechanism for initiation and progression of Parkinson's disease? Acta Neuropathol. Commun. 5:71.

McNamara, N. B., and Miron, V. E. (2020). Microglia in developing white matter and perinatal brain injury. Neurosci. Lett. 714:134539. doi: 10.1016/j.neulet. 2019.134539

Merkus, F. W. H. M., and van den Berg, M. P. (2007). Can nasal drug delivery bypass the blood-brain barrier? Drugs R D 8, 133-144. doi: 10.2165/00126839200708030-00001

Miller, S. P., Cozzio, C. C., Goldstein, R. B., Ferriero, D. M., Partridge, J. C., Vigneron, D. B., et al. (2003). Comparing the diagnosis of white matter injury in premature newborns with serial MR imaging and transfontanel ultrasonography findings. AJNR Am. J. Neuroradiol. 24, 1661-1669.

Mo, M., Wang, S., Zhou, Y., Li, H., and Wu, Y. (2016). Mesenchymal stem cell subpopulations: phenotype, property and therapeutic potential. Cell. Mol. Life Sci. 73, 3311-3321. doi: 10.1007/s00018-016-2229-7

Mondello, S., Thelin, E. P., Shaw, G., Salzet, M., Visalli, C., Cizkova, D., et al. (2018). Extracellular vesicles: pathogenetic, diagnostic and therapeutic value in traumatic brain injury. Expert Rev. Proteomics 15, 451-461. doi: 10.1080/ 14789450.2018.1464914

Morton, M. C., and Feliciano, D. M. (2016). Neurovesicles in brain development. Cell. Mol. Neurobiol. 36, 409-416. doi: 10.1007/s10571-015-0297-0

Morton, M. C., Neckles, V. N., Seluzicki, C. M., Holmberg, J. C., and Feliciano, D. M. (2018). Neonatal subventricular zone neural stem cells release extracellular vesicles that act as a microglial morphogen. Cell Rep. 23, 78-89. doi: 10.1016/j.celrep.2018.03.037

Murphy, D. E., De Jong, O. G., Brouwer, M., Wood, M. J., Lavieu, G., Schiffelers, R. M., et al. (2019). Extracellular vesicle-based therapeutics: natural versus engineered targeting and trafficking. Exp. Mol. Med. 51, 1-12.

Murphy, S., Lim, R., Dickinson, H., Acharya, R., Rosli, S., Jenkin, G., et al. (2010). Human amnion epithelial cells prevent bleomycin-induced lung injury and preserve lung function. Cell Transplant. 19, 909-923. doi: 10.3727/ 096368910x543385

Murray, A. L., Thompson, D. K., Pascoe, L., Leemans, A., Inder, T. E., Doyle, L. W., et al. (2016). White matter abnormalities and impaired attention abilities in children born very preterm. Neuroimage $124,75-84$. doi: 10.1016/j. neuroimage.2015.08.044

Murray, D. M., Boylan, G. B., Ryan, C. A., and Connolly, S. (2009). Early EEG findings in hypoxic-ischemic encephalopathy predict outcomes at 2 years. Pediatrics 124, e459-e467.
Naeye, R. L., and Lin, H. M. (2001). Determination of the timing of fetal brain damage from hypoxemia-ischemia. Am. J. Obstet. Gynecol. 184, 217-224. doi: 10.1067/mob.2001.108996

Nakamura, K., Arimura, K., Nishimura, A., Tachibana, M., Yoshikawa, Y., Makihara, N., et al. (2016). Possible involvement of basic FGF in the upregulation of PDGFR $\beta$ in pericytes after ischemic stroke. Brain Res. 1630, 98-108. doi: 10.1016/j.brainres.2015.11.003

Nishida-Aoki, N., Izumi, Y., Takeda, H., Takahashi, M., Ochiya, T., and Bamba, T. (2020). Lipidomic analysis of cells and extracellular vesicles from high- and low-metastatic triple-negative breast cancer. Metabolites 10:67. doi: 10.3390/ metabo10020067

Ohmichi, T., Mitsuhashi, M., Tatebe, H., Kasai, T., Ali El-Agnaf, O. M., and Tokuda, T. (2019). Quantification of brain-derived extracellular vesicles in plasma as a biomarker to diagnose Parkinson's and related diseases. Parkinsonism Relat. Disord. 61, 82-87. doi: 10.1016/j.parkreldis.2018. 11.021

Ophelders, D. R. M. G., Gussenhoven, R., Klein, L., Jellema, R. K., Westerlaken, R. J. J., Hütten, M. C., et al. (2020). Preterm brain injury, antenatal triggers, and therapeutics: timing is key. Cells 9:1871. doi: 10.3390/cells9081871

Ophelders, D. R., Wolfs, T. G., Jellema, R. K., Zwanenburg, A., Andriessen, P., Delhaas, T., et al. (2016). Mesenchymal stromal cell-derived extracellular vesicles protect the fetal brain after hypoxia-ischemia. Stem Cells Transl. Med. 5, 754-763. doi: 10.5966/sctm.2015-0197

Oskoui, M., Coutinho, F., Dykeman, J., Jetté, N., and Pringsheim, T. (2013). An update on the prevalence of cerebral palsy: a systematic review and meta-analysis. Dev. Med. Child Neurol. 55, 509-519. doi: 10.1111/dmcn.1 2080

Pardridge, W. M. (2012). Drug transport across the blood-brain barrier. J. Cereb. Blood Flow Metab. 32, 1959-1972.

Park, H., Park, H., Mun, D., Kang, J., Kim, H., Kim, M., et al. (2018). Extracellular vesicles derived from hypoxic human mesenchymal stem cells attenuate GSK3 $\beta$ expression via miRNA-26a in an ischemia-reperfusion injury model. Yonsei Med. J. 59, 736-745. doi: 10.3349/ymj.2018.59.6.736

Patel, D. B., Gray, K. M., Santharam, Y., Lamichhane, T. N., Stroka, K. M., and Jay, S. M. (2017). Impact of cell culture parameters on production and vascularization bioactivity of mesenchymal stem cell-derived extracellular vesicles. Bioeng. Transl. Med. 2, 170-179. doi: 10.1002/btm2.10065

Patel, D. B., Santoro, M., Born, L. J., Fisher, J. P., and Jay, S. M. (2018). Towards rationally designed biomanufacturing of therapeutic extracellular vesicles: impact of the bioproduction microenvironment. Biotechnol. Adv. 36, 2051-2059. doi: 10.1016/j.biotechadv.2018.09.001

Penn, A. A., Gressens, P., Fleiss, B., Back, S. A., and Gallo, V. (2016). Controversies in preterm brain injury. Neurobiol. Dis. 92, 90-101. doi: 10.1016/j.nbd.2015. 10.012

Pierson, C. R., Folkerth, R. D., Billiards, S. S., Trachtenberg, F. L., Drinkwater, M. E., Volpe, J. J., et al. (2007). Gray matter injury associated with periventricular leukomalacia in the premature infant. Acta Neuropathol. 114, 619-631. doi: 10.1007/s00401-007-0295-5

Pimentel-Coelho, P. M., and Mendez-Otero, R. (2010). Cell therapy for neonatal hypoxic-ischemic encephalopathy. Stem Cells Dev. 19, 299-310.

Pimentel-Coelho, P. M., Magalhaes, E. S., Lopes, L. M., Deazevedo, L. C., Santiago, M. F., and Mendez-Otero, R. (2010). Human cord blood transplantation in a neonatal rat model of hypoxic-ischemic brain damage: functional outcome related to neuroprotection in the striatum. Stem Cells Dev. 19, 351-358. doi: $10.1089 /$ scd. 2009.0049

Platt, M. J., Cans, C., Johnson, A., Surman, G., Topp, M., Torrioli, M. G., et al. (2007). Trends in cerebral palsy among infants of very low birthweight $(<1500$ g) or born prematurely ( $<32$ weeks) in 16 European centres: a database study. Lancet 369, 43-50. doi: 10.1016/s0140-6736(07)60030-0

Polanco, J. C., Scicluna, B. J., Hill, A. F., and Götz, J. (2016). Extracellular vesicles isolated from the brains of $\mathrm{rTg} 4510$ mice seed tau protein aggregation in a threshold-dependent manner. J. Biol. Chem. 291, 12445-12466. doi: 10.1074/ jbc.m115.709485

Polglase, G. R., Miller, S. L., Barton, S. K., Baburamani, A. A., Wong, F. Y., Aridas, J. D., et al. (2012). Initiation of resuscitation with high tidal volumes causes cerebral hemodynamic disturbance, brain inflammation and injury in preterm lambs. PLoS One 7:e39535. doi: 10.1371/journal.pone.0039535 
Pollet, H., Conrard, L., Cloos, A. S., and Tyteca, D. (2018). Plasma membrane lipid domains as platforms for vesicle biogenesis and shedding? Biomolecules 8:94. doi: 10.3390/biom8030094

Prada, I., Gabrielli, M., Turola, E., Iorio, A., D’arrigo, G., Parolisi, R., et al. (2018). Glia-to-neuron transfer of miRNAs via extracellular vesicles: a new mechanism underlying inflammation-induced synaptic alterations. Acta Neuropathol. 135, 529-550. doi: 10.1007/s00401-017-1803-x

Rajendran, L., Honsho, M., Zahn, T. R., Keller, P., Geiger, K. D., Verkade, P., et al. (2006). Alzheimer's disease $\beta$-amyloid peptides are released in association with exosomes. Proc. Natl. Acad. Sci. U.S.A. 103, 11172-11177. doi: 10.1073/pnas. 0603838103

Ranasinghe, H. S., Scheepens, A., Sirimanne, E., Mitchell, M. D., Williams, C. E., and Fraser, M. (2012). Inhibition of MMP-9 activity following hypoxic ischemia in the developing brain using a highly specific inhibitor. Dev. Neurosci. 34, 417-427. doi: 10.1159/000343257

Ranasinghe, H. S., Williams, C. E., Christophidis, L. J., Mitchell, M. D., Fraser, M., and Scheepens, A. (2009). Proteolytic activity during cortical development is distinct from that involved in hypoxic ischemic injury. Neuroscience 158, $732-744$.

Ratajczak, M. Z., Kucia, M., Jadczyk, T., Greco, N. J., Wojakowski, W., Tendera, M., et al. (2012). Pivotal role of paracrine effects in stem cell therapies in regenerative medicine: can we translate stem cell-secreted paracrine factors and microvesicles into better therapeutic strategies? Leukemia 26, 1166-1173. doi: 10.1038/leu.2011.389

Reddy, K., Mallard, C., Guan, J., Marks, K., Bennet, L., Gunning, M., et al. (1998). Maturational Change in the Cortical Response to Hypoperfusion Injury in the Fetal Sheep. Pediatr. Res. 43, 674-682. doi: 10.1203/00006450-19980500000017

Rezaie, P., and Dean, A. (2002). Periventricular leukomalacia, inflammation and white matter lesions within the developing nervous system. Neuropathology 22, 106-132. doi: 10.1046/j.1440-1789.2002.00438.x

Robertson, C. M., Watt, M. J., and Yasui, Y. (2007). Changes in the prevalence of cerebral palsy for children born very prematurely within a populationbased program over 30 years. JAMA 297, 2733-2740. doi: 10.1001/jama.297.24. 2733

Robey, P. (2017). "Mesenchymal stem cells": fact or fiction, and implications in their therapeutic use. F1000Res 6:524. doi: 10.12688/f1000research.10955.1

Rutherford, M. A., Supramaniam, V., Ederies, A., Chew, A., Bassi, L., Groppo, M., et al. (2010). Magnetic resonance imaging of white matter diseases of prematurity. Neuroradiology 52, 505-521.

Saint-Pol, J., Gosselet, F., Duban-Deweer, S., Pottiez, G., and Karamanos, Y. (2020). Targeting and crossing the blood-brain barrier with extracellular vesicles. Cells 9:851. doi: 10.3390/cells9040851

Santner-Nanan, B., Peek, M. J., Mccullagh, P., and Nanan, R. (2005). Therapeutic potential of stem cells in perinatal medicine. Aust. N. Z. J. Obstet. Gynaecol. 45, $102-107$.

Schmahmann, J., and Pandya, D. (2006). Fibre Pathways of the Brain. New York, NY: Oxford University Press.

Schneider, J., and Miller, S. P. (2019). Preterm brain Injury: white matter injury. Handb. Clin. Neurol. 162, 155-172. doi: 10.1016/b978-0-444-64029-1.00007-2

Seifert, H. A., Leonardo, C. C., Hall, A. A., Rowe, D. D., Collier, L. A., Benkovic, S. A., et al. (2012). The spleen contributes to stroke induced neurodegeneration through interferon gamma signaling. Metab. Brain Dis. 27, 131-141. doi: 10. 1007/s11011-012-9283-0

Shankaran, S., Laptook, A. R., Tyson, J. E., Ehrenkranz, R. A., Bann, C. M., Das, A., et al. (2012). Evolution of encephalopathy during whole body hypothermia for neonatal hypoxic-ischemic encephalopathy. J. Pediatr. 160, 567-572.e563.

Sharma, P., Mesci, P., Carromeu, C., Mcclatchy, D. R., Schiapparelli, L., Yates, J. R., et al. (2019). Exosomes regulate neurogenesis and circuit assembly. Proc. Natl. Acad. Sci. U.S.A. 116:16086. doi: 10.1073/pnas.1902513116

Sharma, P., Schiapparelli, L., and Cline, H. T. (2013). Exosomes function in cellcell communication during brain circuit development. Curr. Opin. Neurobiol. 23, 997-1004. doi: 10.1016/j.conb.2013.08.005

Sheller-Miller, S., Lei, J., Saade, G., Salomon, C., Burd, I., and Menon, R. (2016). Feto-Maternal trafficking of exosomes in murine pregnancy models. Front. Pharmacol. 7:432. doi: 10.3389/fphar.2016.00432

Shu, J., Mo, S., Wen, Q., Qin, Y., and Jiang, L. (2018). Study on immune regulation of bone marrow mesenchymal stem cell-derived exosomes in preterm infants with brain injury. Nanosci. Nanotechnol. Lett. 10, 1598-1605. doi: 10.1166/nnl. 2018.2824

Simpson, R. J., Jensen, S. S., and Lim, J. W. (2008). Proteomic profiling of exosomes: current perspectives. Proteomics 8, 4083-4099. doi: 10.1002/pmic.200800109

Sisa, C., Kholia, S., Naylor, J., Herrera Sanchez, M. B., Bruno, S., Deregibus, M. C., et al. (2019). Mesenchymal stromal cell derived extracellular vesicles reduce hypoxia-ischaemia induced perinatal brain injury. Front. Physiol. 10:282. doi: 10.3389/fphys.2019.00282

Skiöld, B., Wu, Q., Hooper, S. B., Davis, P. G., Mcintyre, R., Tolcos, M., et al. (2014). Early detection of ventilation-induced brain injury using magnetic resonance spectroscopy and diffusion tensor imaging: an in vivo study in preterm lambs. PLoS One 9:e95804. doi: 10.1371/journal.pone.0095804

Skotland, T., Sagini, K., Sandvig, K., and Llorente, A. (2020). An emerging focus on lipids in extracellular vesicles. Adv. Drug Deliv. Rev. 159, 308-321. doi: 10.1016/j.addr.2020.03.002

Skranes, J., Løhaugen, G. C., Martinussen, M., Håberg, A., Brubakk, A. M., and Dale, A. M. (2013). Cortical surface area and IQ in very-low-birth-weight (VLBW) young adults. Cortex 49, 2264-2271. doi: 10.1016/j.cortex.2013.06.001

Smith, Z. J., Lee, C., Rojalin, T., Carney, R. P., Hazari, S., Knudson, A., et al. (2015). Single exosome study reveals subpopulations distributed among cell lines with variability related to membrane content. J. Extracell. Vesicles 4:28533. doi: 10.3402/jev.v4.28533

Somiya, M., Yoshioka, Y., and Ochiya, T. (2017). Drug delivery application of extracellular vesicles; insight into production, drug loading, targeting, and pharmacokinetics. AIMS Bioeng. 4, 73-92.

Spaull, R., Mcpherson, B., Gialeli, A., Clayton, A., Uney, J., Heep, A., et al. (2019). Exosomes populate the cerebrospinal fluid of preterm infants with posthaemorrhagic hydrocephalus. Int. J. Dev. Neurosci. 73, 59-65. doi: 10.1016/j. ijdevneu.2019.01.004

Stoll, B. J., Hansen, N. I., Adams-Chapman, I., Fanaroff, A. A., Hintz, S. R., Vohr, B., et al. (2004). Neurodevelopmental and growth impairment among extremely low-birth-weight infants with neonatal infection. JAMA 292, 2357-2365. doi: 10.1001/jama.292.19.2357

Sun, L. Q., Guo, G. L., Zhang, S., and Yang, L. L. (2018). Effects of MicroRNA-592$5 \mathrm{p}$ on hippocampal neuron injury following hypoxic-ischemic brain damage in neonatal mice - involvement of PGD2/DP and PTGDR. Cell. Physiol. Biochem. 45, 458-473. doi: 10.1159/000486923

Takenouchi, T., Tsukimoto, M., Iwamaru, Y., Sugama, S., Sekiyama, K., Sato, M., et al. (2015). Extracellular ATP induces unconventional release of glyceraldehyde-3-phosphate dehydrogenase from microglial cells. Immunol. Lett. 167, 116-124. doi: 10.1016/j.imlet.2015.08.002

Taylor, D. D., and Shah, S. (2015). Methods of isolating extracellular vesicles impact down-stream analyses of their cargoes. Methods 87, 3-10. doi: 10.1016/j.ymeth. 2015.02.019

Thery, C., Witwer, K. W., Aikawa, E., Alcaraz, M. J., Anderson, J. D., Andriantsitohaina, R., et al. (2018). Minimal information for studies of extracellular vesicles 2018 (MISEV2018): a position statement of the International Society for Extracellular Vesicles and update of the MISEV2014 guidelines. J. Extracell. Vesicles 7:1535750.

Théry, C., Zitvogel, L., and Amigorena, S. (2002). Exosomes: composition, biogenesis and function. Nat. Rev. Immunol. 2, 569-579. doi: 10.1038/nri855

Thomi, G., Joerger-Messerli, M., Haesler, V., Muri, L., Surbek, D., and Schoeberlein, A. (2019a). Intranasally administered exosomes from umbilical cord stem cells have preventive neuroprotective effects and contribute to functional recovery after perinatal brain injury. Cells 8:855. doi: 10.3390/ cells 8080855

Thomi, G., Surbek, D., Haesler, V., Joerger-Messerli, M., and Schoeberlein, A. (2019b). Exosomes derived from umbilical cord mesenchymal stem cells reduce microglia-mediated neuroinflammation in perinatal brain injury. Stem Cell Res. Ther. 10:105.

Thompson, A. G., Gray, E., Heman-Ackah, S. M., Mager, I., Talbot, K., Andaloussi, S. E., et al. (2016). Extracellular vesicles in neurodegenerative disease pathogenesis to biomarkers. Nat. Rev. Neurol. 12, 346-357. doi: 10.1038/ nrneurol.2016.68

Tietje, A., Maron, K. N., Wei, Y., and Feliciano, D. M. (2014). Cerebrospinal fluid extracellular vesicles undergo age dependent declines and contain known and novel non-coding RNAs. PLoS One 9:e113116. doi: 10.1371/journal.pone. 0113116 
Valadi, H., Ekström, K., Bossios, A., Sjöstrand, M., Lee, J. J., and Lötvall, J. O. (2007). Exosome-mediated transfer of mRNAs and microRNAs is a novel mechanism of genetic exchange between cells. Nat. Cell Biol. 9, 654-659. doi: 10.1038/ncb1596 van den Broek, M. P., Groenendaal, F., Egberts, A. C., and Rademaker, C. M. (2010). Effects of hypothermia on pharmacokinetics and pharmacodynamics: a systematic review of preclinical and clinical studies. Clin. Pharmacokinet. 49, 277-294. doi: 10.2165/11319360-000000000-00000

van Haastert, I. C., Groenendaal, F., Uiterwaal, C. S., Termote, J. U., Van Der HeideJalving, M., and Eijsermans, M. J. (2011). Decreasing incidence and severity of cerebral palsy in prematurely born children. J. Pediatr. 159, 86-91.e81.

van Tilborg, E., De Theije, C. G. M., Van Hal, M., Wagenaar, N., De Vries, L. S., Benders, M. J., et al. (2018). Origin and dynamics of oligodendrocytes in the developing brain: implications for perinatal white matter injury. Glia 66, 221-238. doi: 10.1002/glia.23256

van Tilborg, E., Heijnen, C. J., Benders, M. J., Van Bel, F., Fleiss, B., Gressens, P., et al. (2016). Impaired oligodendrocyte maturation in preterm infants: potential therapeutic targets. Prog. Neurobiol. 136, 28-49. doi: 10.1016/j.pneurobio.2015. 11.002

van Velthoven, C. T., Kavelaars, A., and Heijnen, C. J. (2012). Mesenchymal stem cells as a treatment for neonatal ischemic brain damage. Pediatr. Res. 71, 474-481. doi: 10.1038/pr.2011.64

van Velthoven, C. T., Kavelaars, A., Van Bel, F., and Heijnen, C. J. (2010). Mesenchymal stem cell treatment after neonatal hypoxic-ischemic brain injury improves behavioral outcome and induces neuronal and oligodendrocyte regeneration. Brain Behav. Immun. 24, 387-393. doi: 10.1016/j.bbi.2009.10.017

Vannucci, R. C., and Vannucci, S. J. (1997). A model of perinatal hypoxic-ischemic brain damage. Ann. N. Y. Acad. Sci. 835, 234-249.

Vawda, R., Woodbury, J., Covey, M., Levison, S. W., and Mehmet, H. (2007). Stem cell therapies for perinatal brain injuries. Semin. Fetal Neonatal Med. 12, 259-272. doi: 10.1016/j.siny.2007.02.003

Vincer, M. J., Allen, A. C., Joseph, K. S., Stinson, D. A., Scott, H., and Wood, E. (2006). Increasing prevalence of cerebral palsy among very preterm infants: a population-based study. Pediatrics 118, e1621-e1626.

Vogel, A., Upadhya, R., and Shetty, A. K. (2018). Neural stem cell derived extracellular vesicles: attributes and prospects for treating neurodegenerative disorders. EBioMedicine 38, 273-282. doi: 10.1016/j.ebiom.2018.11.026

Volpe, J. J., Kinney, H. C., Jensen, F. E., and Rosenberg, P. A. (2011). The developing oligodendrocyte: key cellular target in brain injury in the premature infant. Int. J. Dev. Neurosci. 29, 423-440. doi: 10.1016/j.ijdevneu.2011.02.012

Walsh, W. (2015). Report of a pilot study of Cooling four preterm infants 32-35 weeks gestation with HIE. J. Neonatal Perinatal Med. 8, 47-51. doi: 10.3233/ npm- 15814078

Wang, A. Y., Chughtai, A. A., Lui, K., and Sullivan, E. A. (2017). Morbidity and mortality among very preterm singletons following fertility treatment in Australia and New Zealand, a population cohort study. BMC Pregnancy Childbirth 17:50. doi: 10.1186/s12884-017-1235-6

Wang, J.-X., Jia, X.-J., Liu, Y., Dong, J.-H., Ren, X.-M., Xu, O., et al. (2020). Silencing of miR-17-5p suppresses cell proliferation and promotes cell apoptosis by directly targeting PIK3R1 in laryngeal squamous cell carcinoma. Cancer Cell Int. 20:14.

Wang, X., Rousset, C. I., Hagberg, H., and Mallard, C. (2006). Lipopolysaccharideinduced inflammation and perinatal brain injury. Semin. Fetal Neonatal Med. 11, 343-353. doi: 10.1016/j.siny.2006.04.002

Wassink, G., Davidson, J. O., Dhillon, S. K., Fraser, M., Galinsky, R., Bennet, L., et al. (2017). Partial white and grey matter protection with prolonged infusion of recombinant human erythropoietin after asphyxia in preterm fetal sheep. J. Cereb. Blood Flow Metab. 37, 1080-1094. doi: 10.1177/0271678x16650455

Webb, R. L., Kaiser, E. E., Jurgielewicz, B. J., Spellicy, S., Scoville, S. L., Thompson, T. A., et al. (2018a). Human neural stem cell extracellular vesicles improve recovery in a porcine model of ischemic stroke. Stroke 49, 1248-1256. doi: 10.1161/strokeaha.117.020353

Webb, R. L., Kaiser, E. E., Scoville, S. L., Thompson, T. A., Fatima, S., Pandya, C., et al. (2018b). Human neural stem cell extracellular vesicles improve tissue and functional recovery in the murine thromboembolic stroke model. Transl. Stroke Res. 9, 530-539. doi: 10.1007/s12975-017-0599-2

Webster, M. J., Herman, M. M., Kleinman, J. E., and Shannon Weickert, C. (2006). $\mathrm{BDNF}$ and trkB mRNA expression in the hippocampus and temporal cortex during the human lifespan. Gene Expr. Patterns 6, 941-951. doi: 10.1016/j. modgep.2006.03.009

Wiklander, O. P. B., Brennan, M., Lötvall, J., Breakefield, X. O., and El Andaloussi, S. (2019). Advances in therapeutic applications of extracellular vesicles. Sci. Transl. Med. 11:eaav8521. doi: 10.1126/scitranslmed.aav8521

Wiklander, O. P. B., Nordin, J. Z., O’loughlin, A., Gustafsson, Y., Corso, G., Mäger, I., et al. (2015). Extracellular vesicle in vivo biodistribution is determined by cell source, route of administration and targeting. J. Extracell. Vesicles 4:26316. doi: 10.3402/jev.v4.26316

Williams, C. E., Gunn, A. J., Synek, B., and Gluckman, P. D. (1990). Delayed seizures occurring with hypoxic-ischemic encephalopathy in the fetal sheep. Pediatr. Res. 27, 561-565. doi: 10.1203/00006450-199006000-00004

Williams, C. E., Gunn, A., and Gluckman, P. D. (1991). Time course of intracellular edema and epileptiform activity following prenatal cerebral ischemia in sheep. Stroke 22, 516-521. doi: 10.1161/01.str.22.4.516

Willms, E., Cabañas, C., Mäger, I., Wood, M. J. A., and Vader, P. (2018). Extracellular vesicle heterogeneity: subpopulations, isolation techniques, and diverse functions in cancer progression. Front. Immunol. 9:738. doi: 10.3389/ fimmu.2018.00738

Willms, E., Johansson, H. J., Mäger, I., Lee, Y., Blomberg, K. E. M., Sadik, M., et al. (2016). Cells release subpopulations of exosomes with distinct molecular and biological properties. Sci. Rep. 6:22519.

Wurzelmann, M., Romeika, J., and Sun, D. (2017). Therapeutic potential of brainderived neurotrophic factor (BDNF) and a small molecular mimics of BDNF for traumatic brain injury. Neural Regen. Res. 12,7-12. doi: 10.4103/1673-5374. 198964

Wyss-Coray, T., Feng, L., Masliah, E., Ruppe, M. D., Lee, H. S., Toggas, S. M., et al. (1995). Increased central nervous system production of extracellular matrix components and development of hydrocephalus in transgenic mice overexpressing transforming growth factor-beta 1. Am. J. Pathol. 147, 53-67.

Xin, D., Li, T., Chu, X., Ke, H., Yu, Z., Cao, L., et al. (2020). Mesenchymal stromal cell-derived extracellular vesicles modulate microglia/macrophage polarization and protect the brain against hypoxia-ischemic injury in neonatal mice by targeting delivery of miR-21a-5p. Acta Biomater. 113, 597-613. doi: 10.1016/ j.actbio. 2020.06 .037

Xin, H., Li, Y., Buller, B., Katakowski, M., Zhang, Y., Wang, X., et al. (2012). Exosome-mediated transfer of miR-133b from multipotent mesenchymal stromal cells to neural cells contributes to neurite outgrowth. Stem Cells 30, 1556-1564. doi: 10.1002/stem.1129

Xin, H., Li, Y., Cui, Y., Yang, J. J., Zhang, Z. G., and Chopp, M. (2013a). Systemic administration of exosomes released from mesenchymal stromal cells promote functional recovery and neurovascular plasticity after stroke in rats. J. Cereb. Blood Flow Metab. 33, 1711-1715. doi: 10.1038/jcbfm.2013.152

Xin, H., Li, Y., Liu, Z., Wang, X., Shang, X., Cui, Y., et al. (2013b). MiR-133b promotes neural plasticity and functional recovery after treatment of stroke with multipotent mesenchymal stromal cells in rats via transfer of exosomeenriched extracellular particles. Stem Cells 31, 2737-2746. doi: 10.1002/stem. 1409

Yan, S., Zhang, H., Xie, W., Meng, F., Zhang, K., Jiang, Y., et al. (2017). Altered microRNA profiles in plasma exosomes from mesial temporal lobe epilepsy with hippocampal sclerosis. Oncotarget 8, 4136-4146. doi: 10.18632/ oncotarget.13744

Yang, J., Zhang, X., Chen, X., Wang, L., and Yang, G. (2017). Exosome mediated delivery of miR-124 promotes neurogenesis after ischemia. Mol. Ther. Nucleic Acids 7, 278-287. doi: 10.1016/j.omtn.2017.04.010

Yao, H., Ma, R., Yang, L., Hu, G., Chen, X., Duan, M., et al. (2014). MiR-9 promotes microglial activation by targeting MCPIP1. Nat. Commun. 5:4386.

Yeo, K. T., Lee, Q. Y., Quek, W. S., Wang, Y. A., Bolisetty, S., Lui, K., et al. (2015). Trends in morbidity and mortality of extremely preterm multiple gestation newborns. Pediatrics 136, 263-271. doi: 10.1542/peds.2014-4075

Yoon, Y. J., Kim, O. Y., and Gho, Y. S. (2014). Extracellular vesicles as emerging intercellular communicasomes. BMB Rep. 47, 531-539. doi: 10.5483/bmbrep. 2014.47.10.164

Zagrean, A. M., Hermann, D. M., Opris, I., Zagrean, L., and Popa-Wagner, A. (2018). Multicellular crosstalk between exosomes and the neurovascular unit after cerebral ischemia. Therapeutic implications. Front. Neurosci. 12:811. doi: 10.3389/fnins.2018.00811 
Zhang, G., Zhu, Z., Wang, H., Yu, Y., Chen, W., Waqas, A., et al. (2020). Exosomes derived from human neural stem cells stimulated by interferon gamma improve therapeutic ability in ischemic stroke model. J. Adv. Res. 24, 435-445.

Zhang, L., Graf, I., Kuang, Y., Zheng, X., Haupt, M., Majid, A., et al. (2021). Neural progenitor cell-derived extracellular vesicles enhance blood-brain barrier integrity by NF- $\kappa \mathrm{B}$ (Nuclear Factor- $\kappa \mathrm{B}$ )-dependent regulation of ABCB1 (ATPbinding cassette transporter B1) in stroke mice. Arterioscler. Thromb. Vasc. Biol. 41, 1127-1145. doi: 10.1161/atvbaha.120.315031

Zhang, Y., Chopp, M., Meng, Y., Katakowski, M., Xin, H., Mahmood, A., et al. (2015). Effect of exosomes derived from multipluripotent mesenchymal stromal cells on functional recovery and neurovascular plasticity in rats after traumatic brain injury. J. Neurosurg. 122, 856-867. doi: 10.3171/2014.11.jns 14770

Zwanenburg, A., Jellema, R. K., Jennekens, W., Ophelders, D., Vullings, R., Van Hunnik, A., et al. (2013). Heart rate-mediated blood pressure control in preterm fetal sheep under normal and hypoxic-ischemic conditions. Pediatr. Res. 73, 420-426. doi: 10.1038/pr.2013.15
Conflict of Interest: The authors declare that the research was conducted in the absence of any commercial or financial relationships that could be construed as a potential conflict of interest.

Publisher's Note: All claims expressed in this article are solely those of the authors and do not necessarily represent those of their affiliated organizations, or those of the publisher, the editors and the reviewers. Any product that may be evaluated in this article, or claim that may be made by its manufacturer, is not guaranteed or endorsed by the publisher.

Copyright (c) 2021 Gamage and Fraser. This is an open-access article distributed under the terms of the Creative Commons Attribution License (CC BY). The use, distribution or reproduction in other forums is permitted, provided the original author(s) and the copyright owner(s) are credited and that the original publication in this journal is cited, in accordance with accepted academic practice. No use, distribution or reproduction is permitted which does not comply with these terms. 